University of Texas at El Paso

\title{
DigitalCommons@UTEP
}

Open Access Theses \& Dissertations

2009-01-01

\section{Directional analysis and filtering for dust storm detection in NOAA-AVHRR imagery}

Swapna Janugani

University of Texas at El Paso, sjanugani@miners.utep.edu

Follow this and additional works at: https://digitalcommons.utep.edu/open_etd

Part of the Electrical and Electronics Commons

\section{Recommended Citation}

Janugani, Swapna, "Directional analysis and filtering for dust storm detection in NOAA-AVHRR imagery" (2009). Open Access Theses \& Dissertations. 288.

https://digitalcommons.utep.edu/open_etd/288

This is brought to you for free and open access by DigitalCommons@UTEP. It has been accepted for inclusion in Open Access Theses \& Dissertations by an authorized administrator of DigitalCommons@UTEP. For more information, please contact lweber@utep.edu. 


\section{DIRECTIONAL ANALYSIS AND FILTERING}

\section{FOR DUST STORM DETECTION IN NOAA-AVHRR IMAGERY}

SWAPNA JANUGANI, B.Tech.

Department of Electrical \& Computer Engineering

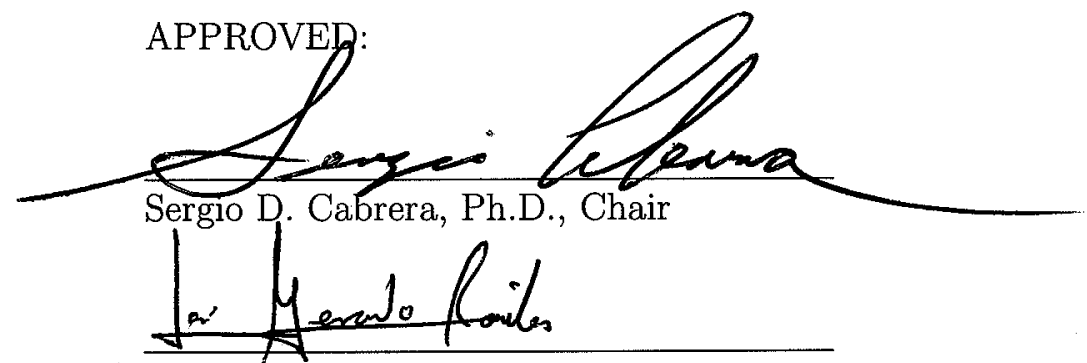

Jose Gerardo Rosiles, Ph.D.

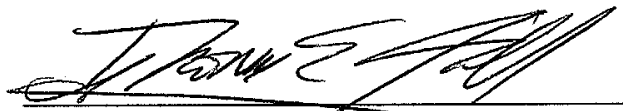

Thomas E. Gill, Ph.D.

Patricia D. Witherspoon, Ph.D.

Dean of the Graduate School 
An Offering

at

HIS Lotus Feet... 


\author{
DIRECTIONAL ANALYSIS AND FILTERING \\ FOR DUST STORM DETECTION IN NOAA-AVHRR IMAGERY
}

by

SWAPNA JANUGANI, B.Tech.

\begin{abstract}
THESIS
Presented to the Faculty of the Graduate School of The University of Texas at El Paso

in Partial Fulfillment

of the Requirements

for the Degree of

MASTER OF SCIENCE

Department of Electrical and Computer Engineering

THE UNIVERSITY OF TEXAS AT EL PASO

January 2009
\end{abstract}




\section{Acknowledgements}

I take this opportunity to express my sincere thanks to my advisor, Dr. Sergio. D. Cabrera, for providing me this opportunity. I appreciate his guidance and support without which this thesis could not be done. I would like to acknowledge the support received from the Texas Instruments Foundation Endowed Scholarship Program.

I would extend my heart-felt gratitude to Mr. Vikram Jayaram for sparing his valuable time, guiding me at every stage and for his continuous encouragement and support.

I would also like to sincerely acknowledge and thank Dr. Jose Gerardo Rosiles for his assistance and serving my committee.

My special thanks to Dr. Thomas E. Gill, from Department of Geosciences, for his timely advice and for serving my committee. I am grateful to Nancy I. Rivera Rivera who has been a great source of help in my venture.

Finally, a deep sense of gratitude is due to my family members and friends, who with their valuable suggestions and moral support, helped me in molding this project into a reality. 


\begin{abstract}
In this research, we propose spatio-spectral processing techniques for the detection of dust storms in 5-band NOAA-AVHRR imagery. The research is mainly focused on locating the dust sources and automatically finding the transport direction of the dust storm. Previous methods that use simple band math analysis have produced promising results but have some drawback in producing consistent results when low SNR images are used. Moreover, in seeking to automate the dust storm detection, the presence of clouds in the vicinity of the dust storm creates a challenge in being able to distinguish these two types of image texture. Our research not only seeks to detect the presence of the dust storm in the imagery, it also attempts to find the directionality and to locate the sources of the dust storm. We propose a spatio-spectral processing scheme with two techniques for this detection scheme - visualization and automation. Visualization technique is intended to locate the dust sources and automation technique is proposed to detect the direction of the dust storm.
\end{abstract}

For visualization technique, image processing algorithms like the spectral-domain $\mathrm{PCA} / \mathrm{MNF}$ transforms, and the spatial-domain k-means unsupervised classification method, are evaluated as tools to help us find dust storms by visual image analysis. For locating the dust sources based on image information, directional filtering is used in combination with edge detectors and spectral-domain classification technique. 
Next, edge detectors like Sobel and Frei-Chen are applied to the selected filtered images for further enhancement of the streaks produced by the directional texture. False color composite images are created to visually enhance the directional streaks to be able to locate the dust sources. The automation technique for finding the direction of the dust storm involves performing the power spectrum analysis on bands 4 and 5 since these wavelengths highlight the absorption and subsequent emission of thermal radiation by the silicate particles in the dust storms. The processing scheme involves block processing applied for power spectrum analysis followed by binary thresholding and morphological enhancement. A local power spectral density analysis is first used to confirm the presence of high directionality information in certain regions of an image. These regions can be determined to be candidate dust storm regions. Binary thresholding is performed on these blocks to enhance the directional texture. Morphological enhancement is done on these binary images to compute their area and orientation. 


\section{Table of Contents}

Page

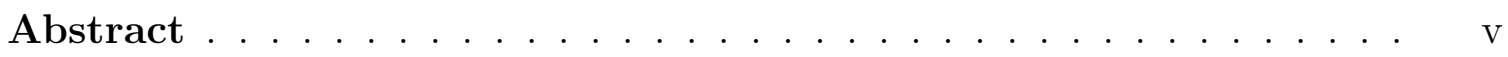

List of Tables ........................ . . . ix

List of Figures . . . . . . . . . . . . . . . . . . . . . 1

Chapter

1 Introduction . . . . . . . . . . . . . . . . . . . . 2

1.1 Multispectral Images . . . . . . . . . . . . . . . . . . . 2

1.2 Dust Storm Detection _. . . . . . . . . . . . . . 5

1.3 Earlier Work. . . . . . . . . . . . . . . . . 6

1.4 Motivation . . . . . . . . . . . . . . . . . . . 7

1.5 Thesis Overview . . . . . . . . . . . . . . . . . 8

\section{Chapter}

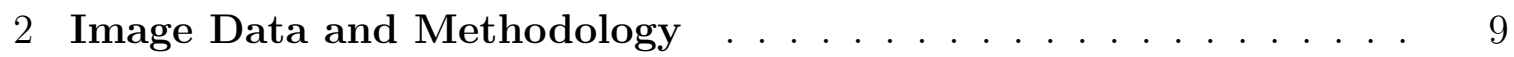

2.1 Satellite Data and Dust Events used in this research . . . . . . . . . 9

2.2 Spatio-spectral processing scheme . . . . . . . . . . . . . 12

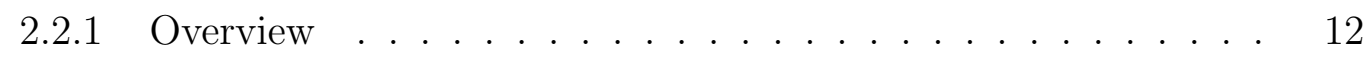

2.2.2 Principal Component Analysis (PCA) . . . . . . . . . 17

2.2.3 Minimum Noise Fraction (MNF) . . . . . . . . . . 24

2.2 .4 k-means Classification . . . . . . . . . . . . . . 29 


\section{Chapter}

3 Visualization technique for dust storm detection . . . . . . . . . . 32

3.1 Block Diagram . . . . . . . . . . . . . . . . . . . 32

3.2 Directional Filtering . . . . . . . . . . . . . . . . 33

3.3 Edge Detection . . . . . . . . . . . . . . . . . . . . . 39

3.3.1 Sobel Edge Detector . . . . . . . . . . . . . . . . . . 41

3.3.2 Frei-Chen Edge Detector . . . . . . . . . . . . . . . 42

3.4 Locating dust sources using False Color Composite images . . . . . . 45

\section{Chapter}

4 Techniques for automating the detection of dust storms . . . . . . 53

4.1 Block Diagram . . . . . . . . . . . . . . . . . . . 53

4.2 Power Spectrum Analysis . . . . . . . . . . . . . . . . 54

4.3 Binary Thresholding . . . . . . . . . . . . . . . . . . . 55

4.4 Morphological Enhancement . . . . . . . . . . . . . . 59

4.5 Quantitative Analysis . . . . . . . . . . . . . . . . . 62

Chapter

5 Experiments and Results . . . . . . . . . . . . . . . 64

Chapter

6 Conclusion and Future Work . . . . . . . . . . . . . . . . . . . 84

Bibliography . . . . . . . . . . . . . . . . . 87

Appendix

A Discussion of MATLAB Implementation . . . . . . . . . . . . . . . . 92

Curriculum Vitae . . . . . . . . . . . . . . . . . . . . . . 95 


\section{List of Tables}

3.1 A 3x3 ENVI directional filter mask at 80 degrees . . . . . . . . 37

3.2 A 3x3 region of an image representing gray-level values . . . . . . . 41

3.3 Horizontal Sobel Operator . . . . . . . . . . . . . . . . 41

3.4 Vertical Sobel Operator . . . . . . . . . . . . . . 42

3.5 Horizontal Frei-Chen Operator . . . . . . . . . . . . . . 44

3.6 Vertical Frei-Chen Operator . . . . . . . . . . . . . . . . . . 44

3.7 Dust storm source locations (latitude, longitudes) from NOAA-16 satellite image for April 15, 2003 20:23 UTC event . . . . . . . . . 51

3.8 Dust storm source locations (latitude, longitudes) from NOAA-16 satellite image for December 15, 2003 19:51 UTC event . . . . . . . . 52

4.1 A $3 \times 3$ structuring element $\mathrm{S} \ldots \ldots$. . . . . . . . . . . . 60

4.2 Binary Image B . . . . . . . . . . . . . . . . . . . . . . . 61

4.3 Closing $B \bullet S \ldots \ldots \ldots \ldots$. . . . . . . . . . . . . . 61 


\section{List of Figures}

1.1 Overview of Remote Sensing system [33] . . . . . . . . . . . . 3

1.2 Layout of Electromagnetic spectrum [39] . . . . . . . . . . . . . . . 3

2.1 Overview of the Sensor-derived Satellite Data used in this research . . 11

2.2 Block Diagram for spatio-spectral processing schemes used for dust storm detection in this thesis . . . . . . . . . . . . . . . 13

2.3 Original band 4 (top-left), band 5 (top-right)and the bandmath (band 4 - band 5) result (bottom) for April 15, 2003 event . . . . . . . . . . 14

2.4 Original band 4 (top-left), band 5 (top-right) and the bandmath (band 4 - band 5) result (bottom) for December 15, 2003 event . . . . . .

2.5 Cropped 512x512 Band 1 (left-side) image and k-means classification (right-side) of cropped 512x512 Band 1 of April 15, 2003 event . . . . 16

2.6 Eigenvalues of PCA transformation of April 15, 2003 event . . . . . . 19

2.7 Component 5 of PCA analysis for April 15, 2003 event . . . . . . . . 20

2.8 Eigenvalues of PCA transformation of December 15, 2003 event . . . 21

2.9 Component Band 5 of PCA analysis for December 15, 2003 event . . 22

2.10 Eigenvalues of MNF transformation of April 15, 2003 event . . . . . . 25

2.11 MNF Component 4 of April 15, 2003 event . . . . . . . . . . . . 26

2.12 Eigenvalues of MNF transformation of December 15, 2003 event . . . 27

2.13 MNF Component 5 of December 15, 2003 event . . . . . . . . . . 28 
2.14 Step-by-step k-means Clustering Algorithm [35] . . . . . . . . . . . . 30

3.1 Block diagram for Visualization . . . . . . . . . . . . . . 33

3.2 Cropped $512 \times 512$ band 4 of April 15, 2003 event . . . . . . . . . . 34

3.3 Cropped $512 \times 512$ band 4 of December 15, 2003 event . . . . . . . . 35

3.4 Directional filtering of cropped band 4 for different angles $20^{\circ}, 30^{\circ}, 40^{\circ}$, $50^{\circ}, 70^{\circ}, 90^{\circ}$ (from left to right) . . . . . . . . . . 37

3.5 Directional filtering of cropped band 4 at 80 degrees for April 15, 2003

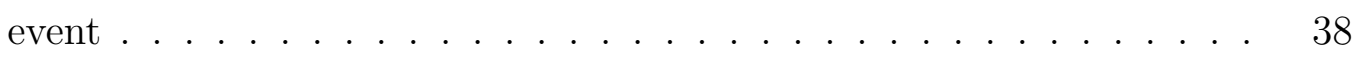

3.6 Calculating Image Gradients . . . . . . . . . . . . . . . . . 40

3.7 Sobel Edge Detection on ENVI directional filtered image of April 15, 2003 at 80 degrees . . . . . . . . . . . . . . . . . 43

3.8 Frei-Chen Edge Detection on ENVI directional filtered image of April 15,2003 at 80 degrees . . . . . . . . . . . . . 46

3.9 False Color Composite image - Band 1, 4, 5 (R, G, B) with marked dust sources for April 15, 2003 event . . . . . . . . . . 47

3.10 False Color Composite image - Band 1, 4, 3 (R, G, B) with marked dust sources for April 15, 2003 event . . . . . . . . . . . 48

3.11 False Color Composite image - Band 1, 3, 4 (R, G, B) with marked dust sources for April 15, 2003 event . . . . . . . . . . . 49

3.12 False Color Composite image - Band 1, 4, 5 (R, G, B) with marked dust sources for December 15, 2003 event . . . . . . . . . . 50

4.1 Block diagram for Automated dust storm detection . . . . . . . . . . 54

4.2 Power spectrum analysis for different topographical features [15] . . 56

4.3 Power spectrum analysis by block processing for 128x128 blocks[15] . 57

4.4 Computation of angle of least inertia . . . . . . . . . . . . 63 
5.1 Cropped 512x512 band 4 of April 15, 2003 event . . . . . . . . .

5.2 Power Spectrum Analysis of April 15, 2003 event (square of magnitude

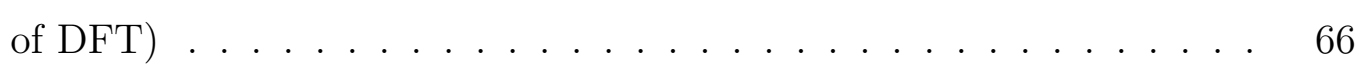

5.3 Binary thresholding of the powerspectrum of April 15, 2003 event . . 67

5.4 Morphological enhancement on the binary thresholded image of April 15,2003 event . . . . . . . . . . . . . . . 69

5.5 Area and Orientation Computations of April 15, 2003 event . . . . . 70

5.6 Power Spectrum Analysis of April 15, 2003 event (square root of magnitude of $\mathrm{DFT}) \ldots \ldots \ldots \ldots 71$

5.7 Binary thresholding of the powerspectrum of April 15, 2003 event . . 72

5.8 Morphological enhancement on the binary thresholded image of April 15,2003 event . . . . . . . . . . . . . . . . 73

5.9 Cropped 512x512 band 4 of December 15, 2003 event . . . . . . . . 74

5.10 Power Spectrum Analysis of December 15, 2003 event . . . . . . . 75

5.11 Binary thresholding of the powerspectrum of December 15, 2003 event 76

5.12 Morphological enhancement on the binary thresholded image of December 15, 2003 event . . . . . . . . . . . . . . . 77

5.13 Area and Orientation Computations of December 15, 2003 event . . . 78

5.14 Bandmath image of April 1, 2003 event . . . . . . . . . . 80

5.15 Original $512 \times 512$ image of April 1, 2003 . . . . . . . . . 81

5.16 k-means classified $512 \times 512$ image of April 1, 2003 . . . . . . . . . 82

5.17 Bandmath result of cropped 512x512 non-dust storm image of December $15,2003 \ldots \ldots \ldots \ldots \ldots$

6.1 Energy of subbands for cropped 512x512 band 4 of April 15, 2003 event 86

A.1 Block diagram for Automated dust storm detection . . . . . . . . . 93 


\section{Chapter 1}

\section{Introduction}

\section{$1.1 \quad$ Multispectral Images}

Remote Sensing is the technology of acquiring information about a planet without actually being in contact with it. This includes the sensing and recording of reflected or emitted energy and processing, analyzing that information [8]. These multispectral remote-sensing images opened up new possibilities for detecting and monitoring atmospheric dust events and tracking the potential environmental risks [3]. Other applications of multispectral images include disaster mitigation, biological and chemical detection, mineral mapping and target detection. Figure 1.1 illustrates the overview of the typical remote-sensing system [36]. Figure 1.2 illustrates the layout of the electromagnetic spectrum.

Multispectral imagery are the multidimensional images, optically acquired in more than one spectral or wavelength interval of the electromagnetic spectrum. Each 2Dimage slice is referred to as a Band. Satellite imagery produces multispectral (multiband) images of the Earth, corresponding to the same physical area and scale, but at different spectral channels in visible and non-visible ranges. Each image taken 


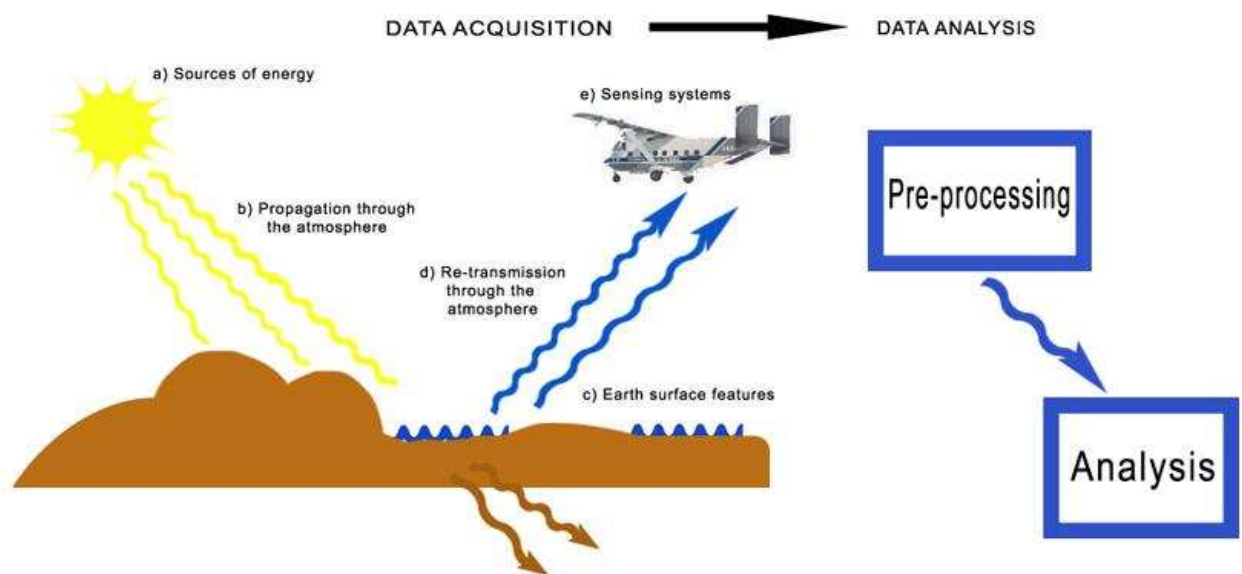

Figure 1.1: Overview of Remote Sensing system [33]

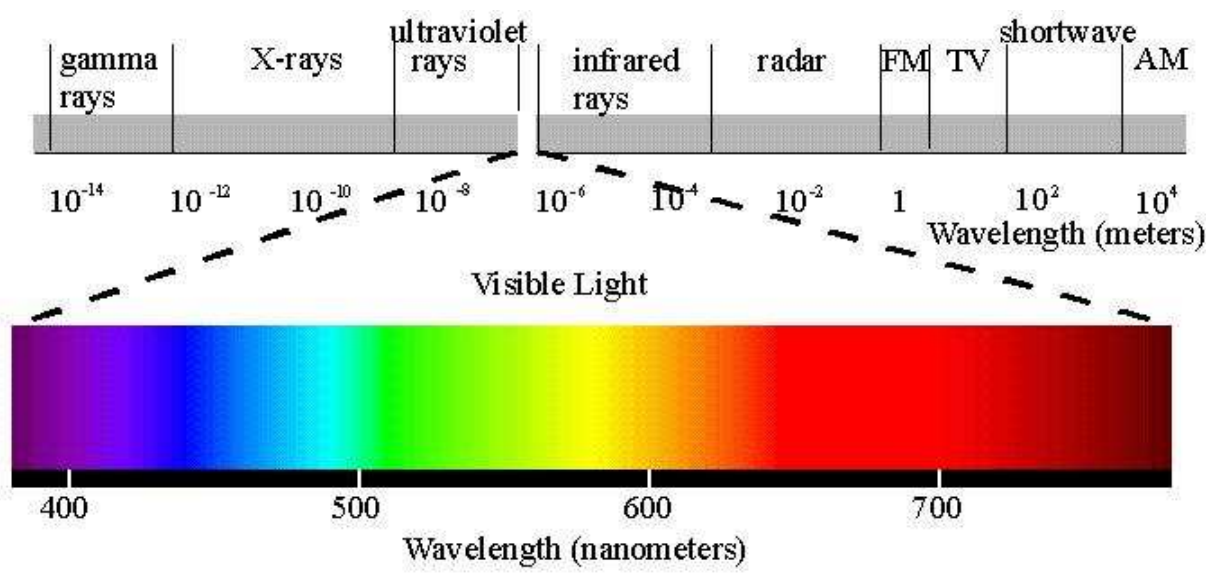

Figure 1.2: Layout of Electromagnetic spectrum [39] 
at a particular spectral band captures certain amount of information about Earth's features. Information is conveyed in spatial, spectral, and temporal variations of those energy fields arising from the Earth's surface. The finite energy field arising from the Earth is divided spatially into pixels. A digital image is an array of pixels depicting spatial distribution of a certain field parameter (such as reflectivity of electromagnetic radiation, emissivity, temperature or some geophysical or topographical elevation). Associated with each pixel is a number denoted as DN (Digital Number), that depicts the average radiance of a relatively small area within a scene, the range of DN values being normally 0 to 255 for an 8-bit per pixel image. The reflected energy in each pixel can be divided into a number of spectral bands. Spectral variation is the primary source of information that allows us to avoid the need for very high spatial resolution [8].

Remote Sensing data obtained from sensors like the Advanced Very High Resolution Radiometer (AVHRR) aboard a National Oceanic and Atmospheric Administration (NOAA) satellite are used to detect and monitor dust events that occur in the atmosphere [3]. Other sensors onboard are the Geostationary Operational Environmental Satellite (GOES) and the Moderate Resolution Imaging Spectroradiometer (MODIS) also have been used for the detection of dust storms. AVHRR and GOES have five spectral bands with a wavelength range from $0.5 \mu \mathrm{m}$ to $12.5 \mu \mathrm{m}$. MODIS has thirty-six spectral bands with a wavelength range of $0.405 \mu \mathrm{m}$ and $14.385 \mu \mathrm{m}$. AVHRR and MODIS are of particular interest in dust storm detection and monitoring because of their relatively high temporal resolutions. The spectral and temporal resolutions of all these sensors are provided in Chapter 2. 


\subsection{Dust Storm Detection}

Dust Storms are major causes of several physical, environmental and economical hazards. Air pollution from dust storms is a significant health hazard for people with respiratory diseases and can adversely impact urban areas. There is a direct link between exposure to high-levels of air-borne particle concentrations and the increase in mortality rate from cardio-vascular, respiratory illness and lung cancer. Timely warnings of dust storms must be initiated in populated regions for health concerns and traffic control [3]. Although several traditional methods for detecting dust storms exist, the use of NOAA-AVHRR images poses difficulties in consistently detecting and distinguishing the dust storm features [4].

In one proposed technique described in this thesis, directional filtering is applied on Band 4 and Band 5 to emphasize the direction of the dust storm. Band $4(10.3 \mu \mathrm{m}$ - $11.3 \mu \mathrm{m})$ and Band $5(11.5 \mu \mathrm{m}-12.50 \mu \mathrm{m})$ highlight the absorption and subsequent emission of thermal radiation by the silicate particles in the dust plume [5]. These wavelengths used for the analysis are similar to the wavelengths of $10.7 \mu \mathrm{m}$ and $12 \mu \mathrm{m}$, (IR brightness temperatures) that Ackerman used in his research [1] [5]. Directional filtering is applied on the sample images at different angles and the resulting image entropy is measured at each angle. The highest entropy at a particular angle signifies the directionality of the dust storm. Sobel and Frei-Chen edge detection further enhances the dust storm visually. Dust Storms viewed in this manner may be difficult to separate from clouds. In order to enhance the dust storm texture and visibly locate the dust sources, false color composite images are produced. A False color composite is an artificial representation of a multispectral image. The display color assignment for any band of a multispectral image is done in an arbitrary manner. In another proposed technique, block processing (dividing the image into blocks and processing each of them independently) is done on these images to automate the detection 
process and to find the direction of the dust storm. The block-based power spectral density analysis with the combination of binary thresholding and morphological enhancements provides quantitative analysis for the automation technique.

\subsection{Earlier Work}

A band math analysis technique has been proposed for dust storm detection in NOAAAVHRR images [1] by Nancy et. al. The difference 'split-window' technique is performed by subtracting band 4 and band 5.The Band 4 and Band 5 were chosen in [1] because $10.7 \mu \mathrm{m}$ and $12 \mu \mathrm{m}$ (IR brightness temperatures) highlighted the absorption and thermal radiation by silicate particles in the dust plume [5]. But, the low Signal-to-Noise Ratio (SNR) of acquired imagery and the presence of clouds in the vicinity of the dust storm may cause detection problems to be even more difficult with band math analysis. Further, the localization of the origin of dust storms was done manually. The absence of automated treatment towards detection and processing led to the research in this thesis.

Hierarchial principal component analysis (HPCA) techniques shown in [6] were significant but computationally challenging and expensive as they involve data fusion both spectrally and spatially from cameras placed at different angles. In most practical scenarios, it is not possible to get the raw data sets of the same scene (same geographical area at a particular time) from cameras placed at different angles.

Koren et al. [32] proposed a dust source detection method using georeferencing of the dust-storm image with the non-dust-storm image of the same location. The limitation of this method is that the technique dependent on the availability of the image at the same location, with identical geometry and resolution. To avoid any false alarms, resulting from clouds or pollution, the image should be as close in time as possible as well. 


\subsection{Motivation}

The main motivation for this research is derived from the work documented in the Master's thesis (Fall 2006) entitled Detection and Characterization of Dust source areas in the Chihuahuan Desert, Southwestern North America written by Nancy I. Rivera Rivera [1]. The methodology developed in [1] primarily used band math analysis as the technique to detect dust storm source areas. Dust sources are located manually by visually locating the dust region pixels. Our research aims at automating this technique for finding the dust sources and direction of the transport of dust storm. Traditional methods like band math analysis, difference of radiation temperature or infrared split-window technique, single band threshold or multi-band combinations have achieved good results in detecting dust storms [1]. However, these techniques have limitations in detecting direction of the dust storm, the visibility index, and the required computational complexity [7]. All these drawbacks led to further research in this area.

In this research, we propose new image processing based methodologies for detecting dust storms in NOAA-AVHRR images. Our proposed techniques include application of directional filtering and edge detection for visual analysis of the dust storms. Directional filtering extracts information about the direction of dust storm. Edge detection techniques like Sobel and Frei-Chen methods on these images resulted in visually clearer and enhanced dust storm images with pronounced directionality information. Further more, different image processing algorithms like Discrete Fourier transform (DFT), binary thresholding and morphological enhancements are applied on the images to automate the dust storm detection and to find the direction of transport and the sources of the dust storm. 


\subsection{Thesis Overview}

The rest of the thesis is organized as follows. Chapter 2 includes the description of the satellite imagery data and the dust events used in this research. This chapter provides an overview of the spatio-spectral methodology for processing the NOAA-AVHRR images to detect the dust storm, to locate it's origin and to find the directionality. This chapter emphasizes the Principal Component Analysis (PCA), Minimum Noise Fraction (MNF) and the k-means classification techniques used for pre-processing the images. The detailed step-by-step procedure for detecting dust storms visually is illustrated in the Chapter 3.

Chapter 3 begins with the analysis methods performed on these multispectral images for visualization of dust storms. Theories on Directional filtering, Sobel and Frei-Chen edge detection algorithms are briefly revisited. This chapter highlights the usage of directional filtering for detecting the direction of dust storms. The technique for locating the origin of dust storms by false color composite images is also explained in this chapter.

In chapter 4 , various techniques used for automating dust storm detection are presented. These include block processing schemes for detecting and finding the direction of a dust storm. Different processing schemes like power spectrum analysis, binary thresholding, morphological enhancements are explained.

Chapter 5 concentrates on the experimental results obtained from visualization and automation techniques. The performance of these processing schemes on three different raw data sets is provided. The results achieved by processing these images in the Environment for Visualizing Images (ENVI) software package and in MATLAB are shown. Chapter 6 is devoted to the conclusions derived from this research and the proposed future work including a preliminary study using the Discrete Wavelet Transform (DWT). 


\section{Chapter 2}

\section{Image Data and Methodology}

This chapter emphasizes the description of the satellite data and techniques like PCA, MNF and k-means clustering as alternative methods for initial detection or verification for bandmath analysis.

\subsection{Satellite Data and Dust Events used in this research}

The Advanced Very High Resolution Radiometer (AVHRR) which is aboard the NOAA - Polar Orbiting Environmental Satellite (POES) is one of the sensors used for aerosol detection and monitoring [3]. Other applications of AVHRR include determining cloud cover and the surface temperature remotely. AVHRR imagery has five spectral bands of $1 \mathrm{~km}$ spatial resolution each.

The current on-orbit operational satellites maintained by NOAA are NOAA-18, 17, 16, 15, 14 and 12 [38]. The first AVHRR was a 4-channel radiometer, launched in October 1978. Later a 5-channel instrument AVHRR-2 was developed which was carried on NOAA-7. NOAA-12 was launched on May 14, 1991. AVHRR-3 which is 
carried on NOAA-15 launched in May 1998 also acquires data in a sixth channel at $1.6 \mu \mathrm{m}$ [33]. The latest NOAA satellites being 16, 17, 18 were launched on September 21, 2000; June 24, 2002 and May 20, 2005 [37].

Other sensors which are of particular interest in dust storm detection and monitoring are the Moderate Resolution Imaging Spectrometer (MODIS) and a sensor onboard the Geostationary Operating Environmental Satellite (GOES). MODIS has thirty-six spectral bands and GOES has five spectral bands. Imagery from GOES are the most suitable to track the time evolution of active and short lived dust storms because of their high temporal resolution [9]. Also,there are three different MODIS sensors - Terra, Aqua and Aura - each on a different satellite covering an area at different times of the day. So, as many as three MODIS images are available per day for the same geographical region.

Images collected by AVHRR and MODIS are of particular interest in dust storm detection because of their relatively higher temporal resolutions (once per day) compared with LANDSAT-type systems (once every 16 days). The nighttime thermal imaging capability of AVHRR and the higher spectral resolution of MODIS can also assist with detecting dust emission and mapping the vulnerability of the landscape to wind erosion [5]. Figure 2.1 provides a summary of relevant sensor-derived satellite data $[1]$.

In this research, we deal mostly with the NOAA-AVHRR multispectral data of two dust events that occurred during April 15th, 2003 (20:23 UTC), and December 15th 2003 (19:51 UTC), a non-dust-storm image from April 1st, 2003 is also used. We choose the NOAA-AVHRR imagery for our analysis because of the ease of data availability. The NOAA-16 raw data sets for the April 15, 2003 and the December 15, 2003 dust events were provided by the Department of the Geological Sciences at UTEP. These two data sets are of sizes 2000x2659 pixels. The other non-dust-storm NOAA15 image i.e., April 1, 2003 data set (Latitude : 22 to 40N, Longitude : -114.0W 


\begin{tabular}{|c|c|c|c|}
\hline Sensor & Number of Bands/Channels & Spatial Resolution & Coverage \\
\hline NOAA-AVHRR & 5 & $1 \mathrm{~km}$ each & daily \\
\hline MODIS & 36 & $\begin{array}{r}\text { Ch } 1-2-250 \mathrm{~m} \\
\text { Ch } 3-8-500 \mathrm{~m} \\
\text { Ch9-36-1 km }\end{array}$ & 1 to 2 days \\
\hline GOES & 5 & $\begin{array}{c}\text { Ch } 1-1 \mathrm{~km} \\
\text { Ch } 2,4,5-4 \mathrm{~km} \\
\text { Ch } 3-8 \mathrm{~km}\end{array}$ & 15 minutes to 3 hours \\
\hline
\end{tabular}

Figure 2.1: Overview of the Sensor-derived Satellite Data used in this research

to $-88.0 \mathrm{~W}$; Start time : 13:28 End time : 13:40) was downloaded from the class (Comprehensive Large Array-data Stewardship System) website [35]. This image is of $2048 \times 1739$ pixels. The (CLASS) is an electronic library of NOAA environmental data and the data sets for any location and date can be downloaded free of cost.

Dust storms can vary in their shape, particle size, distribution and generally tend to show a variable behavior [10]. The differing behavior of the dust and haze in different regions of the electromagnetic spectrum is studied in order to detect and track them [3]. Out of these five spectral bands from NOAA-AVHRR, we can consider mostly band 4 and band 5 for our analysis because Band $4(10.3 \mu \mathrm{m}-11.3 \mu \mathrm{m})$ and Band $5(11.5 \mu \mathrm{m}-12.50 \mu \mathrm{m})$ highlight the absorption and subsequent emission of thermal radiation by the silicate particles in the dust plume [1]. These wavelengths used for the analysis are similar to the wavelengths of $10.7 \mu \mathrm{m}$ and $12 \mu \mathrm{m}$, (IR brightness temperatures) that Ackerman used for aerosol detection [5]. 


\section{$2.2 \quad$ Spatio-spectral processing scheme}

\subsubsection{Overview}

This research is focused on developing a robust approach for locating dust sources and finding dust storm direction in NOAA-AVHRR imagery.

A new spatio-spectral processing scheme is proposed for detecting the presence of dust storm, finding the location of dust sources and determining the dust storm direction for NOAA-AVHRR images. The block diagram shown in figure 2.2 gives an overview of the spatio-spectral processing schemes involved in detecting dust storms by both visualization and by automation techniques. Figure 2.2 also provides an overview of the spatio-spectral processing schemes evaluated in this thesis.

The band math analysis [1]i.e., difference of bands 4 and band 5, is used as an initial step to detect the presence of dust storm. The dark regions in the image indicate the presence of a dust storm region. The presence of clouds in the vicinity of a dust storm would make the problem more difficult. In order to overcome this false alarm, unsupervised k-means classification is performed as a verification step to match the results of band math analysis. Figure 2.3 and 2.4 show the original bands 4 and 5 and the bandmath results (band 4 - band 5) of the April 15, 2003 and December 15, 2003 dust events.

Now, a $512 \times 512$ region containing the dust storm is cropped from all bands in the raw data set. The k-means classification is performed on these bands to verify if it is able to segment the same dust storm region. If the results from band math analysis and k-means are able to segment similar regions, it indicates the potential dust storm presence. So, we approach the next steps of locating the origin of dust storms by visualization technique and finding the direction of the dust storm by automation.

Figure 2.5 shows the result of k-means classification on the cropped $512 \times 512$ band 


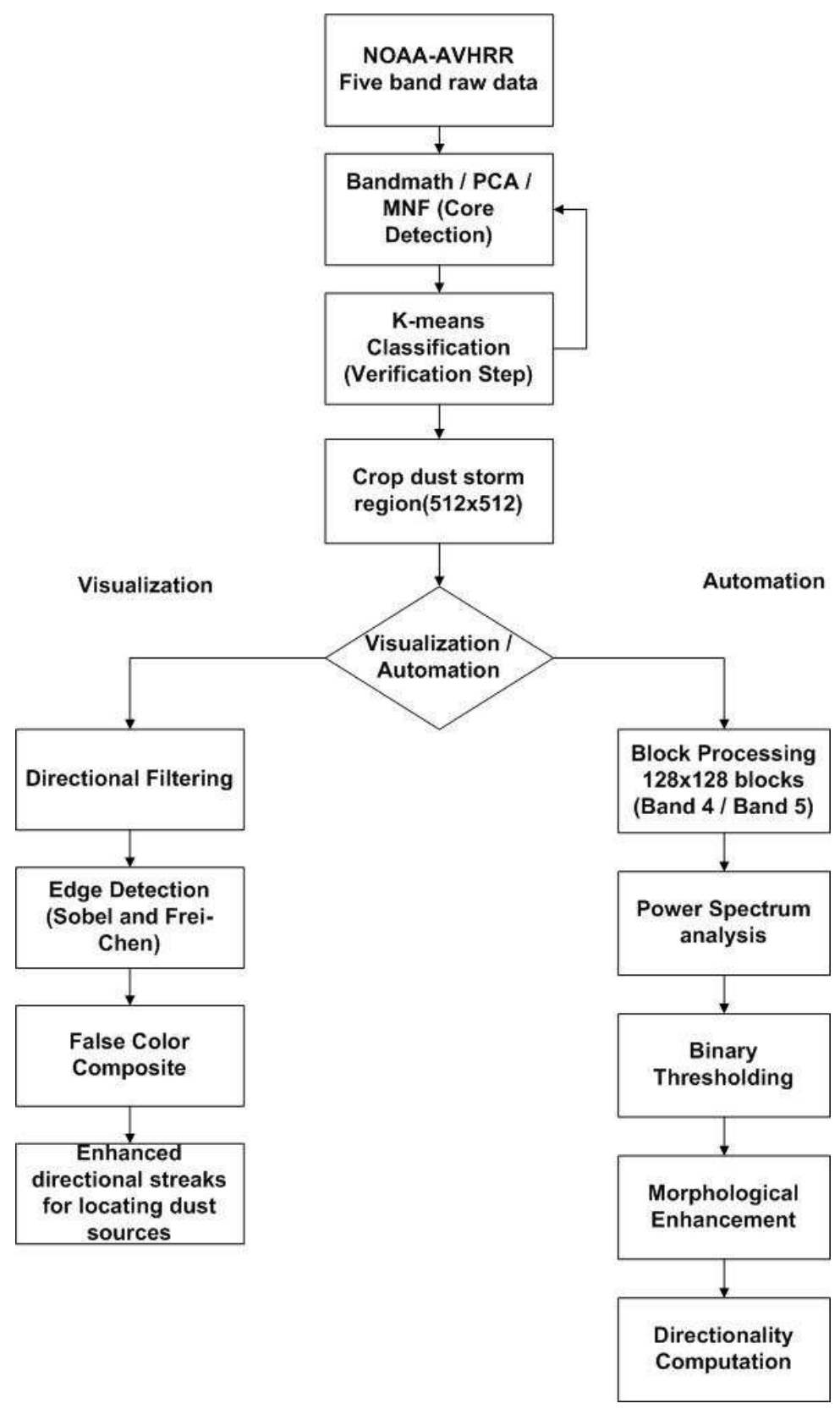

Figure 2.2: Block Diagram for spatio-spectral processing schemes used for dust storm detection in this thesis 

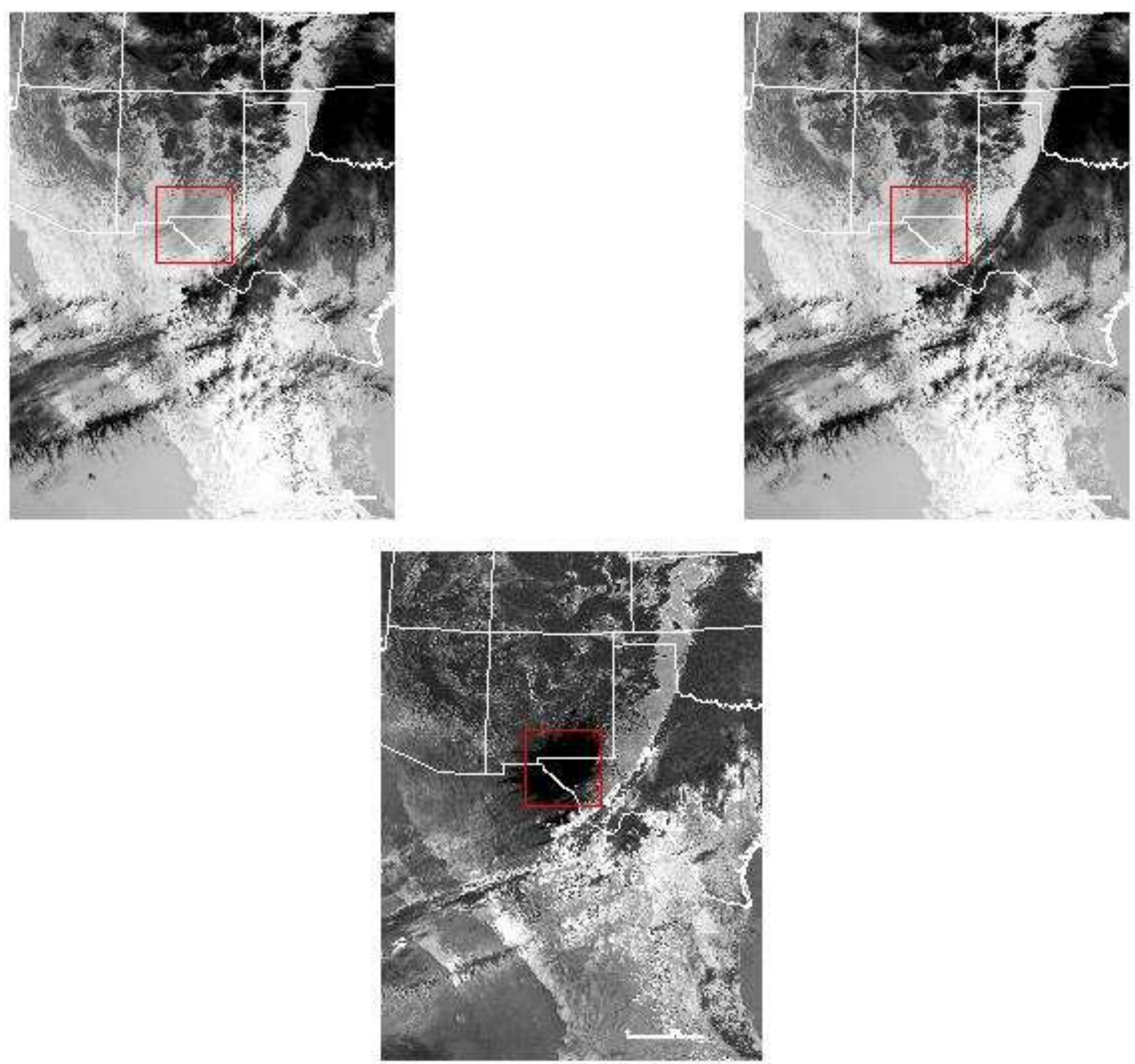

Figure 2.3: Original band 4 (top-left), band 5 (top-right)and the bandmath (band 4 - band 5) result (bottom) for April 15, 2003 event 

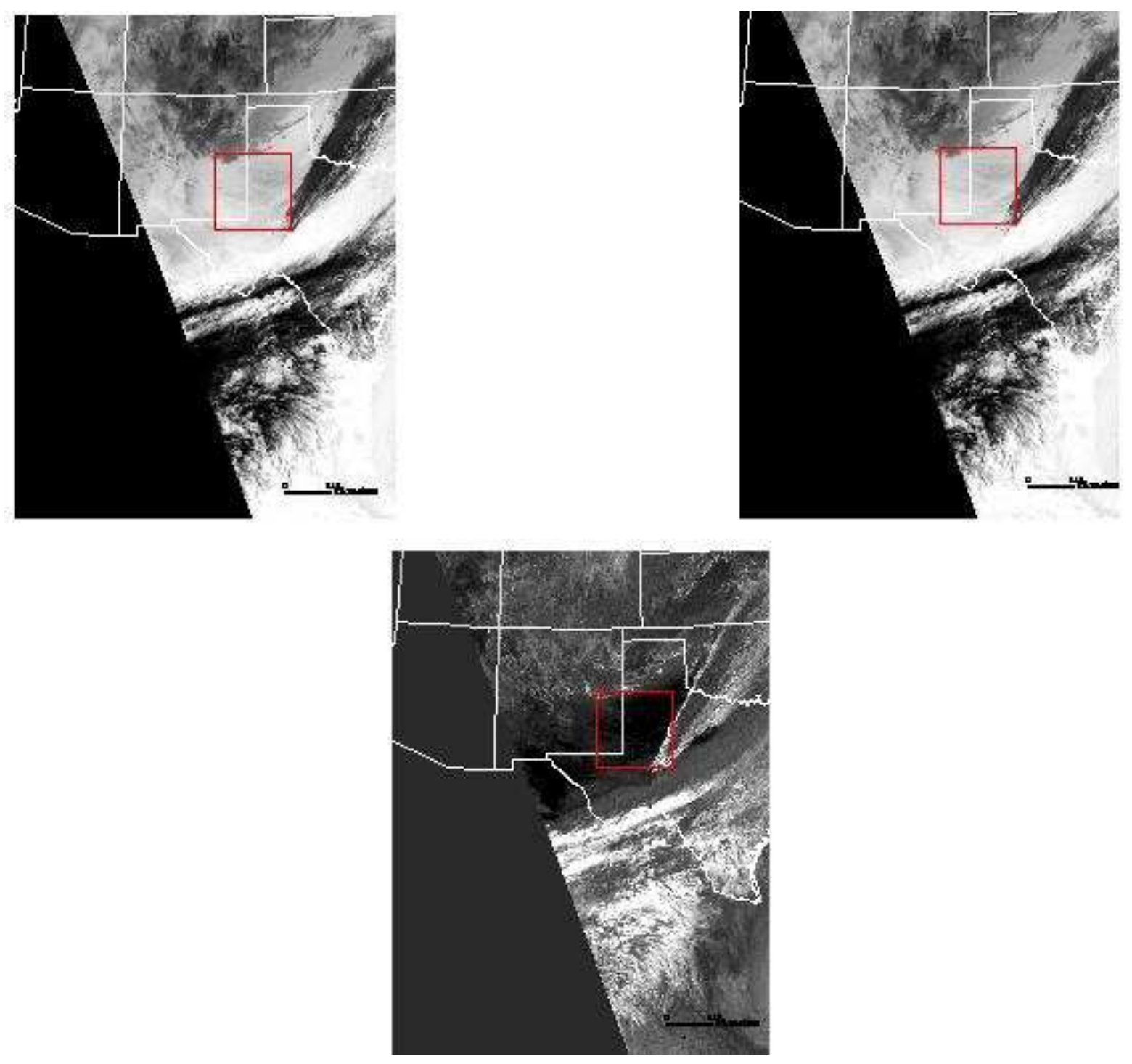

Figure 2.4: Original band 4 (top-left), band 5 (top-right) and the bandmath (band 4 - band 5) result (bottom) for December 15, 2003 event 
1 image of the April 15, 2003 event. Here, the k-means classification is able to segment the dust storm region. So, the next step would be to visually locate the dust storm sources.
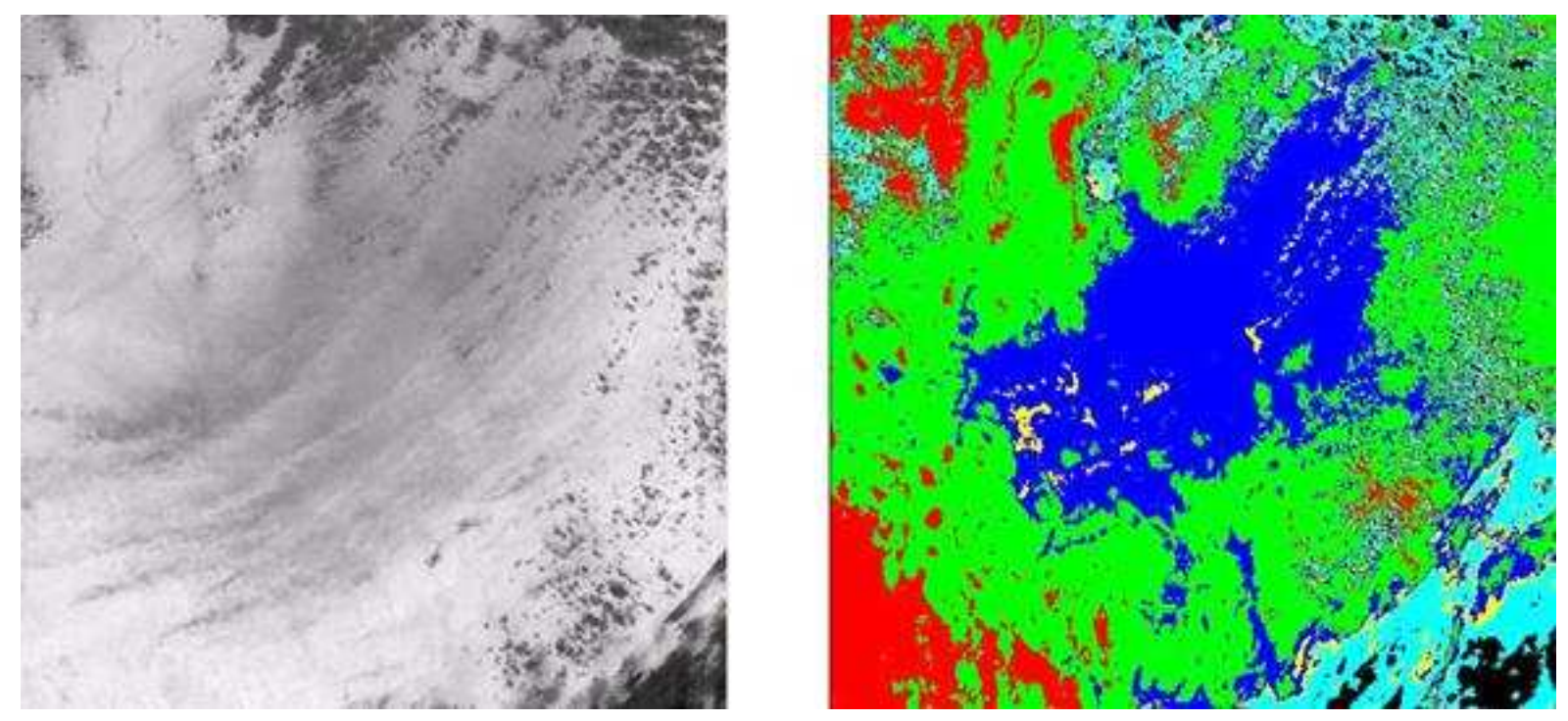

Figure 2.5: Cropped 512x512 Band 1 (left-side) image and k-means classification (right-side) of cropped 512x512 Band 1 of April 15, 2003 event

For visually locating the dust sources, directional filtering is applied on the cropped individual image bands at various angles. Energy and entropy measurements are computed on these directional filtered images to find the prominent direction of motion of the dust storm. Next, edge detectors like Sobel and Frei-Chen are applied to the selected filtered images for further enhancement of the streaks produced by the directional texture. False color composite images are created to differentiate the dust storm image texture and mark the origin points of the resultant directional streaks.

In the automation process, we perform block processing on the cropped $512 \times 512$ 
images of band 4 and band 5. Block processing means dividing an image into blocks and processing them individually and parallelly. The block size chosen is $128 \times 128$ based on the need to have enough resolution in the Discrete Fourier Transform (DFT) images. A local analysis of each Power Spectral Density (PSD) block is used to confirm the presence of high directionality information in certain regions of the image [15]. These regions can be selected as candidate dust storm regions. The presence of a prominent direction in the texture of the candidate dust storm region can be used as further verification of its presence in this automated detection scheme. Binary thresholding based on Otsu's method is performed on these PSD blocks to enhance the directional texture. Otsu's method chooses a threshold to minimize the intraclass variances of the two resulting regions in a binary image [MATLAB 7.5.0 Help]. Morphological enhancements are applied to these resulting binary images to extract the area and orientation of the binary objects that correspond to the largest energy DFT values.

\subsubsection{Principal Component Analysis (PCA)}

Principal component analysis (PCA) or discrete Karhunen-Loève transform (KLT) is a vector space transform used to reduce multi-dimensional data sets to lower dimensions for analysis or visualization. It is also referred as the Hotelling Transform or Proper Orthogonal Decomposition (POD) [24].

PCA is used as a standard tool for enhancement and compression of remotely sensed multispectral data [11]. The PCA transformation has the ability to reduce the dimensionality of a data set while retaining most of the variance by concentrating the majority of the information into the few components. Therefore, it is widely used as a dimension reduction technique. Other applications of PCA include pattern recognition, color contrast enhancement, object tracking and motion estimation. 
The PCA is a linear transformation of a multivariate data set into a new coordinate system. It reorganizes the data in the feature space so that they are no longer correlated. It represents the data in a new coordinate system in which basis vectors follow modes of greatest variance in the data. It is the optimal linear transformation which divides an observed space into orthogonal subspaces with the largest variance. The PCA transforms a number of possibly correlated variables into the same number of uncorrelated variables called principal components. The first principal component accounts for maximum variance in the data, and each succeeding component accounts for as much of the remaining variance as possbile. If the data set has to be approximated in a lower dimension, then lower-order principal components are considered. Thus, the lower order principal components contain the dust storm information, thus considering dust storm as noise, compared to the whole image as signal. This transformation consistently segments the dust storm in the lower order (higher index number) bands. Figure 2.6 and Figure 2.8 show the eigenvalues of PCA transformed images. Principal Component Band 5 is able to segment the dust storm region as shown in Figure 2.7 and Figure 2.9.

The Principal Component Transform is a signal dependent transform since it depends on the auto-covariance matrix; thus the basis has to be computed separately for every input. It is computationally a very expensive transform: no structure can be assumed for the covariance matrix other than being symmetric and positive definite. Therefore, no fast algorithm can be used; the cost of constructing the PCT is of order $\mathrm{O}\left(N^{3}\right)$ [18]. Additional mathematical background is given next, refer [24] for more detail.

Suppose we have a random vector population $\mathrm{X}$, where: $\vec{X}=\left[x_{0}, \ldots, x_{n-1}\right]^{T}$ are $\mathrm{n}$ samples of data.

The mean vector of $\vec{X}$ is denoted by $\mu_{X}=E[X]$, and the covariance matrix $C_{X}$ of $\vec{X}$ is 


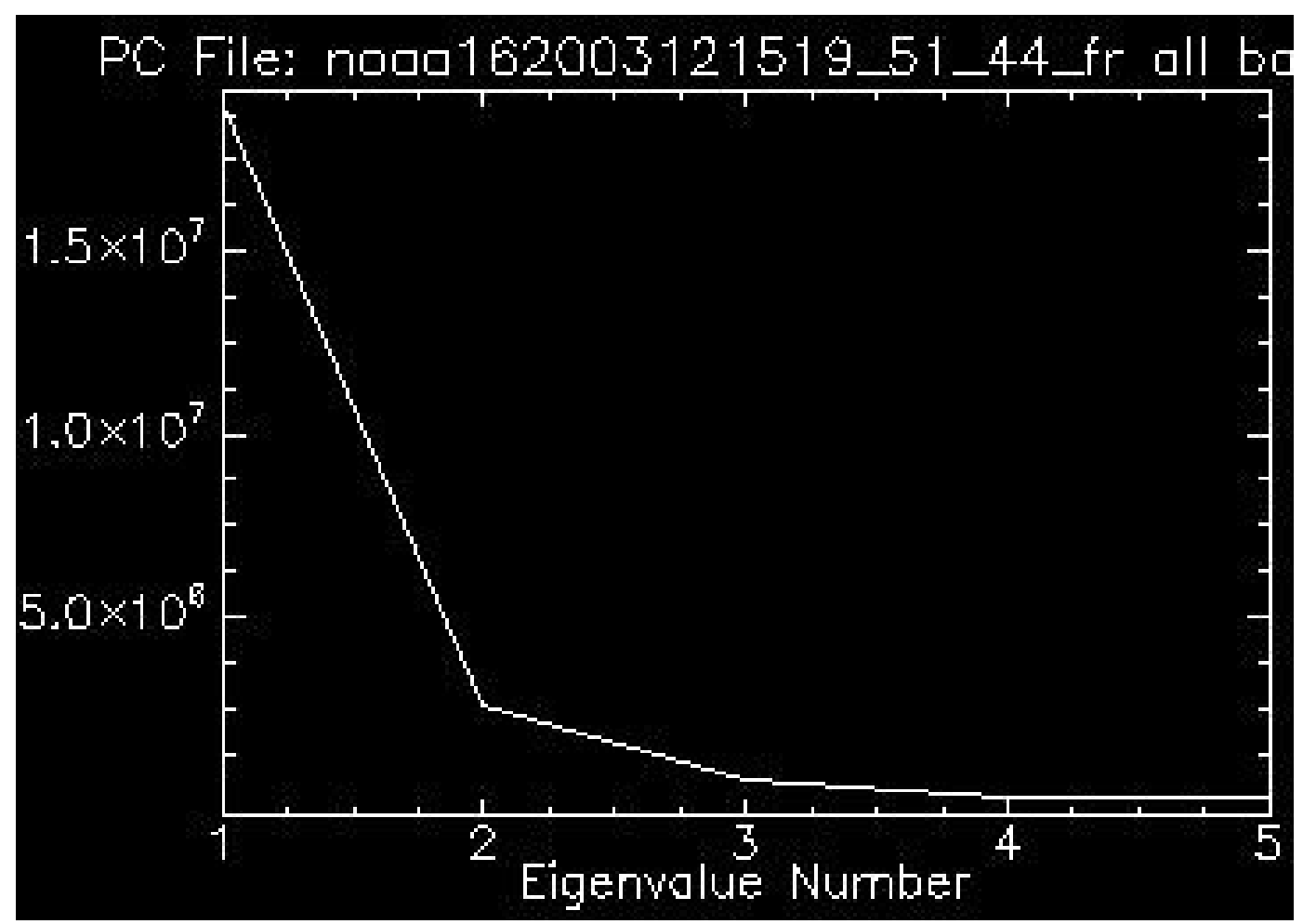

Figure 2.6: Eigenvalues of PCA transformation of April 15, 2003 event 


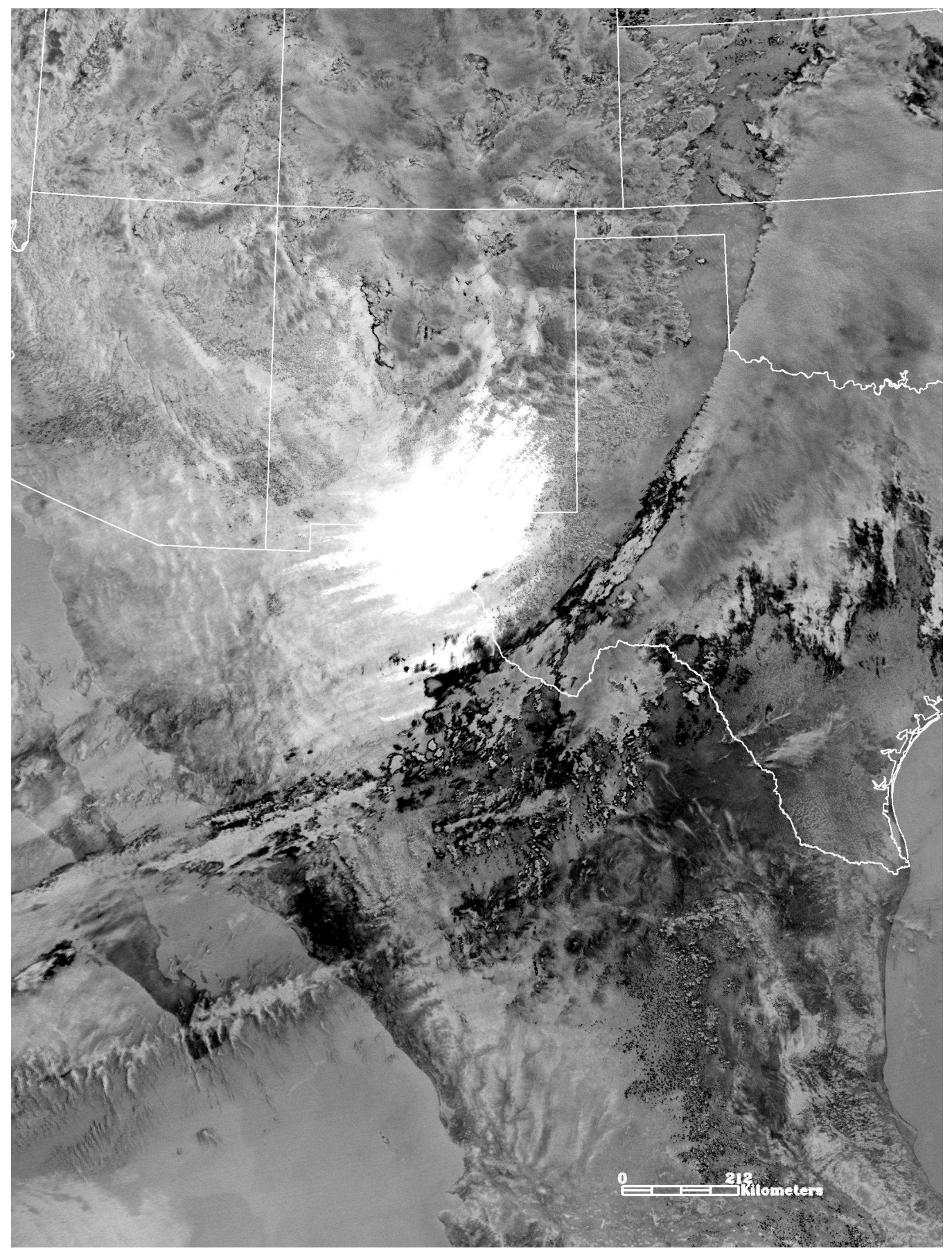

Figure 2.7: Component 5 of PCA analysis for April 15, 2003 event 


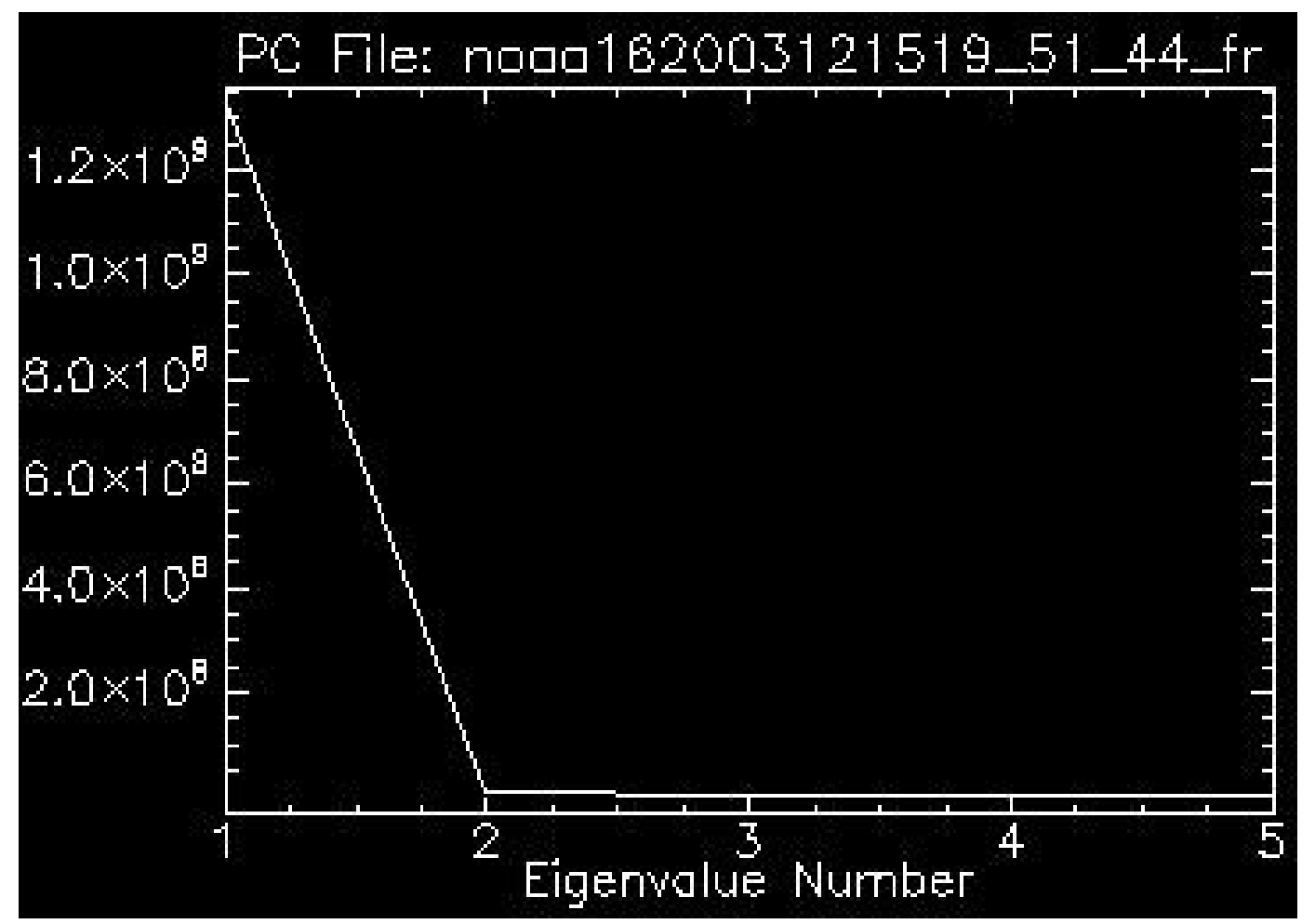

Figure 2.8: Eigenvalues of PCA transformation of December 15, 2003 event 


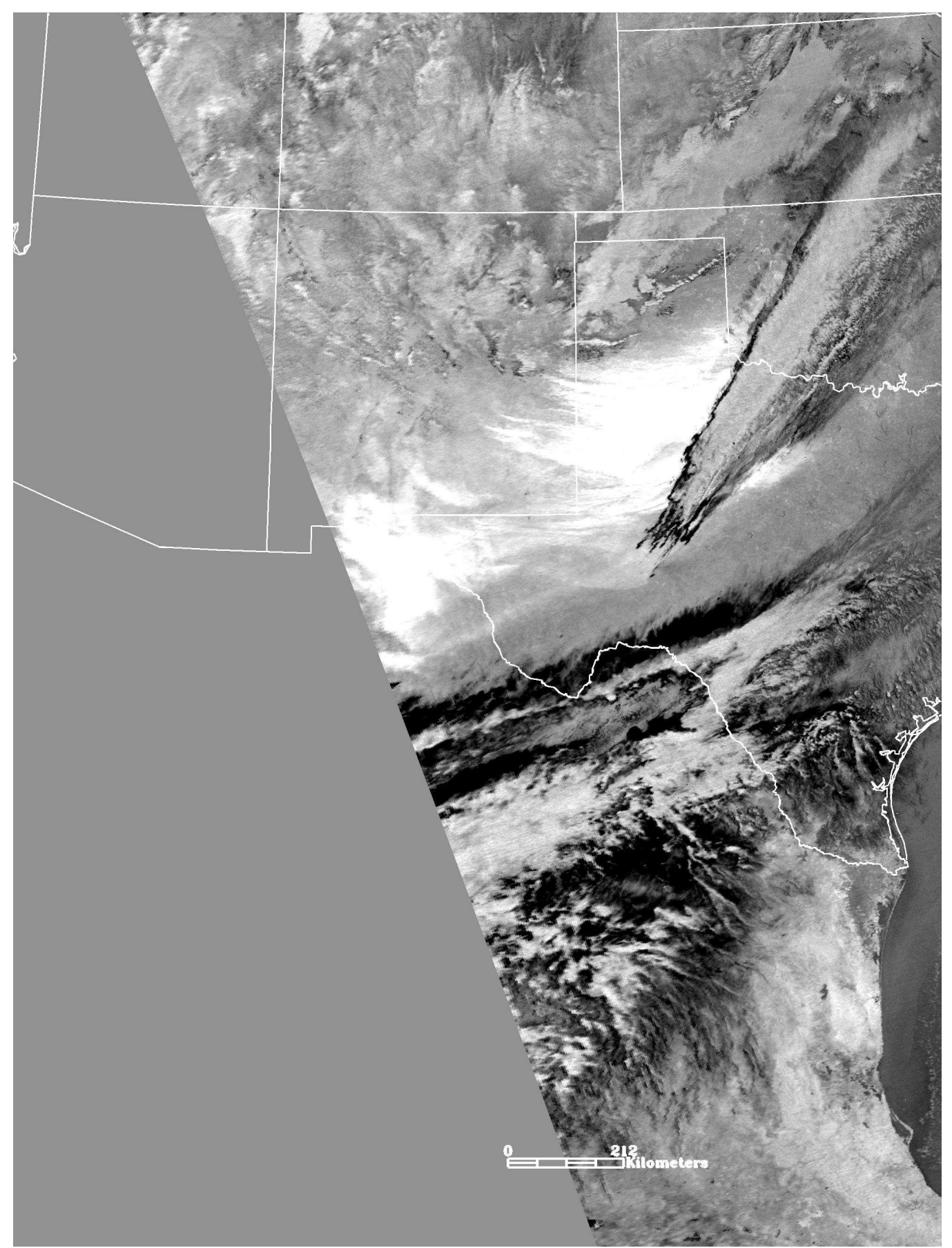

Figure 2.9: Component Band 5 of PCA analysis for December 15, 2003 event 


$$
C_{X} \triangleq E\left[\left(\vec{X}-\overrightarrow{\mu_{X}}\right)\left(\vec{X}-\overrightarrow{\mu_{X}}\right)^{T}\right]=E\left(\vec{X} \vec{X}^{T}\right)-\overrightarrow{\mu_{X}}{\overrightarrow{\mu_{X}}}^{T}=\left[\begin{array}{ccc}
. . & . . & . . \\
. . & \sigma_{i j}^{2} & . \\
. . & . . & . .
\end{array}\right]
$$

The components of $C_{X}$, denoted by $C_{i j}$, represent the covariances between the random variable components $x_{i}$ and $x_{j}$. The component $C_{i i}$ is the variance of component $x_{i}$. If two components $x_{i}$ and $x_{j}$ are uncorrelated, their covariance $C_{i j}=C_{j i}=0$. The covariance matrix is always symmetric, by definition.

From the symmetric covariance matrix, an orthogonal basis is computed by finding eigenvalues and eigenvectors. The eigenvectors $e_{i}$ and the corresponding eigenvalues $\lambda_{i}$ are the solutions of the equation $C_{X} e_{i}=\lambda_{i} e_{i}, i=1, \ldots, n$

These distinct $\lambda_{i}$ values are calculated by finding the solutions of the characteristic equation $\left|C_{X}-\lambda I\right|=0$ where $\mathrm{I}$ is the identity matrix. If the data vector has $\mathrm{n}$ components, the characteristic equation is of order $\mathrm{n}$. The eigenvectors are ordered in the order of descending eigen values. The first eigenvector captures the largest variance of the data.

Let $\mathrm{A}$ be a matrix consisting of eigenvectors of the covariance matrix as the row vectors. By transforming a data vector $\mathrm{X}$, we get $Y=A\left(X-\mu_{X}\right)$, where $\mathrm{y}$ is a point in the orthogonal coordinate system defined by the eigenvectors.

The original data vector $\mathrm{X}$ can be reconstructed from linear combination of the orthogonal basis vectors by $X=A^{T} Y+\mu_{X}$ using the property of an orthogonal matrix: $A^{-1}=A^{T}$.

Instead of using all the eigenvectors of the covariance matrix, the data can be approximated in terms of only few basis vectors of the orthogonal basis. Let $A_{k}$ be the matrix having first $\mathrm{K}$ eigenvectors as rows. Now, the transformation would be $Y=A_{k}\left(X-\mu_{X}\right)$ and $X_{P}=A_{k}^{T} Y+\mu_{X}$

This means that we project the original data vector on the coordinate axes having 
dimension $\mathrm{K}$ and transforming the vector back by linear combination of the subset of basis vectors. This minimizes the mean-square error between the data and the representation with $\mathrm{K}$ eigenvectors. If the data is concentrated in linear subspace, data can be compressed without losing much information and simplifying the representation. By choosing eigenvectors with largest eigenvalues, little information is lost.

\subsubsection{Minimum Noise Fraction (MNF)}

Minimum Noise Fraction (MNF) transform is also referred to as the Noise-Adjusted Transformation (NAPC). The MNF is used to determine the inherent dimensionality of image data, to segregate noise in the data, and to reduce the computational requirements for subsequent processing [12]. The MNF transform is two cascaded principal component transformations. The first transformation is based on the estimated noise covariance matrix. It decorrelates and rescales the noise in the data. This results in transformed data with unit variance noise and no band-to-band correlation. The second transformation is the standard principal component transformation of the noise-whitened data. The spectral bands are ranked based on the signal-to-noise ratios instead of variances as in principal component analysis [11].

Figure 2.10 and Figure 2.12 show the eigenvalues of MNF transformed images. Similar to the principal component transformation, MNF also contains dust storm information in the lower order (higher index number) bands. Here, we observe that dust storm is well segmented in MNF band 4 for April 15, 2003 event and MNF band 5 for the December 15, 2003 event. Figure 2.11 and Figure 2.13 show the MNF transformed images. 


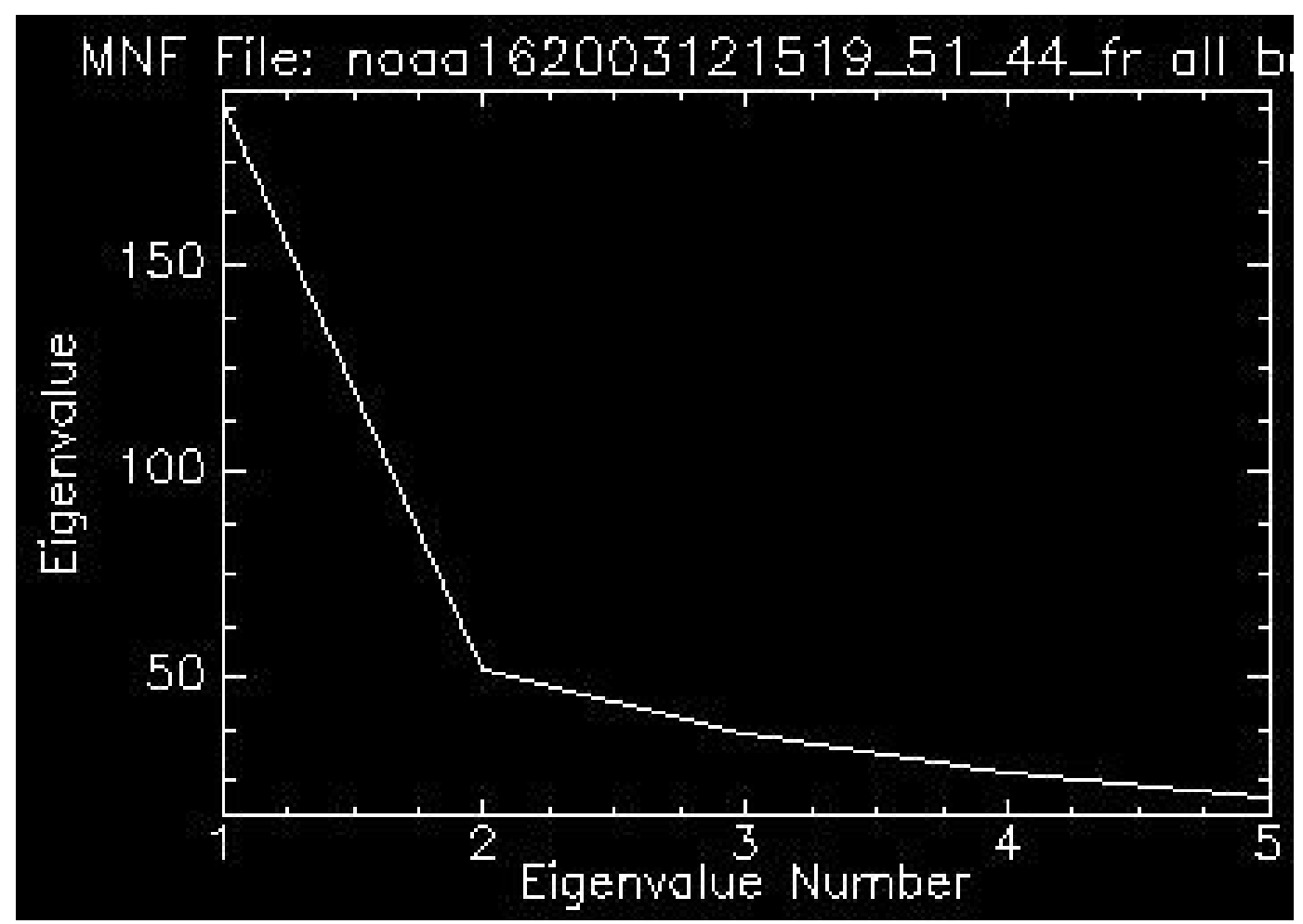

Figure 2.10: Eigenvalues of MNF transformation of April 15, 2003 event 


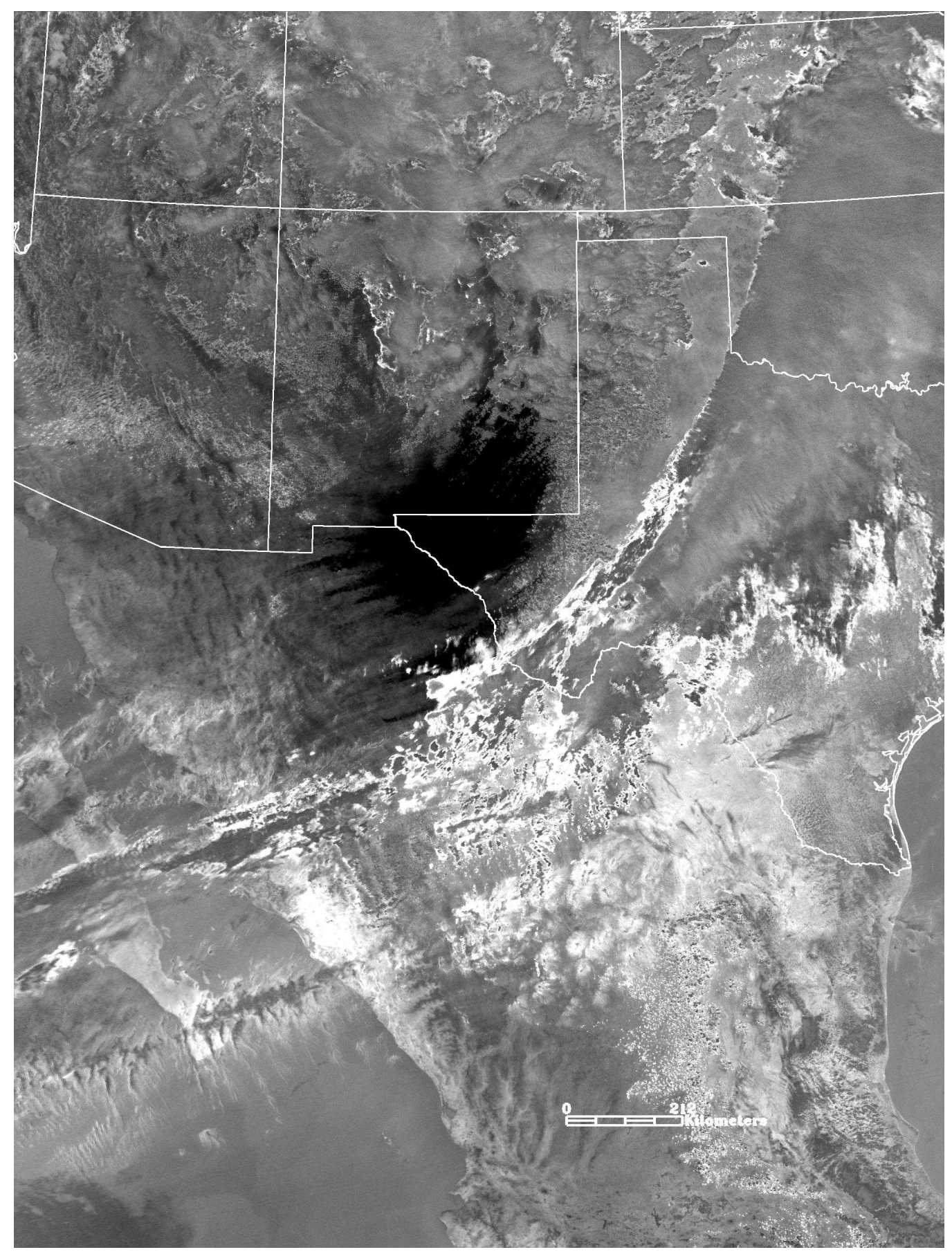

Figure 2.11: MNF Component 4 of April 15, 2003 event 


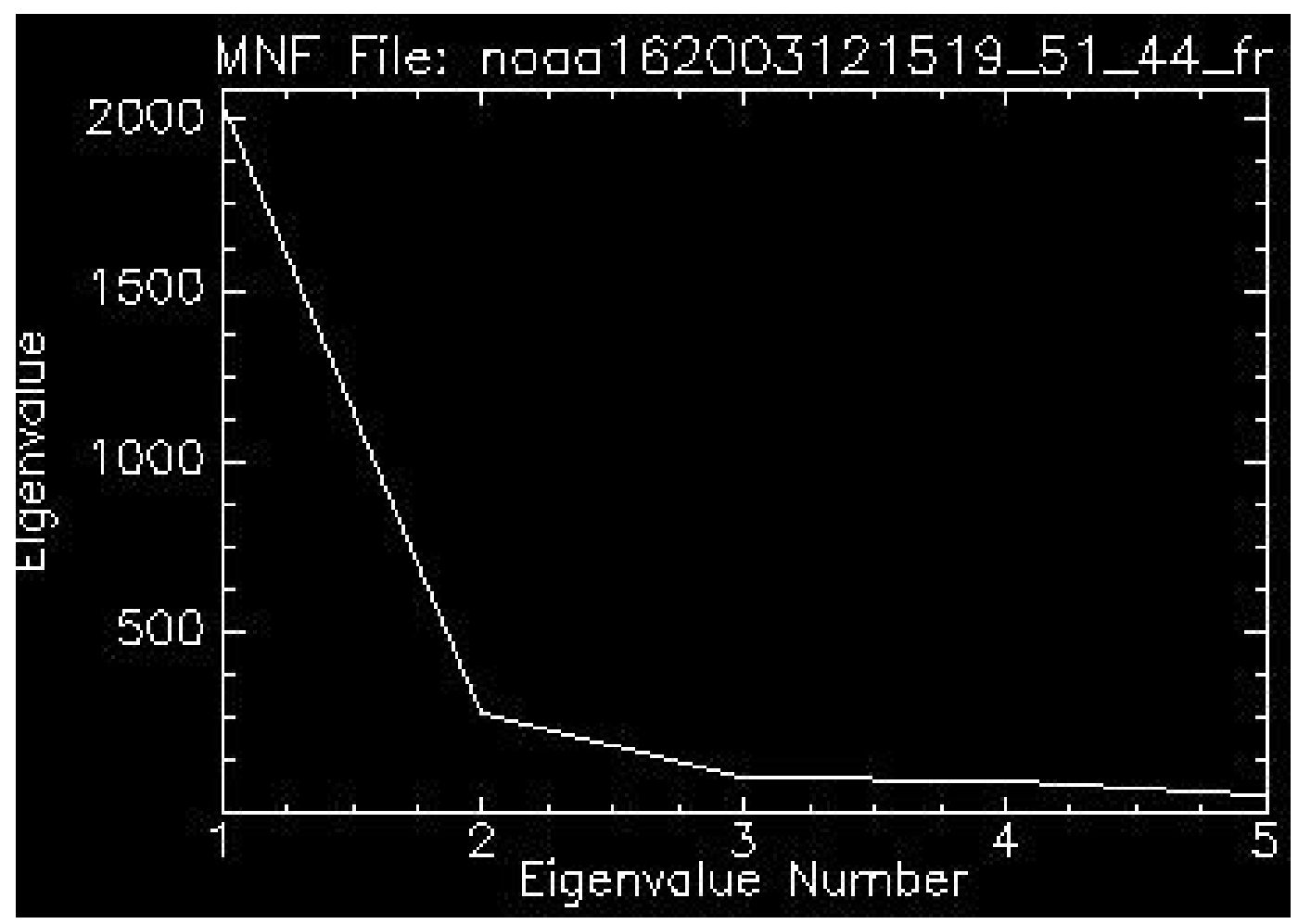

Figure 2.12: Eigenvalues of MNF transformation of December 15, 2003 event 


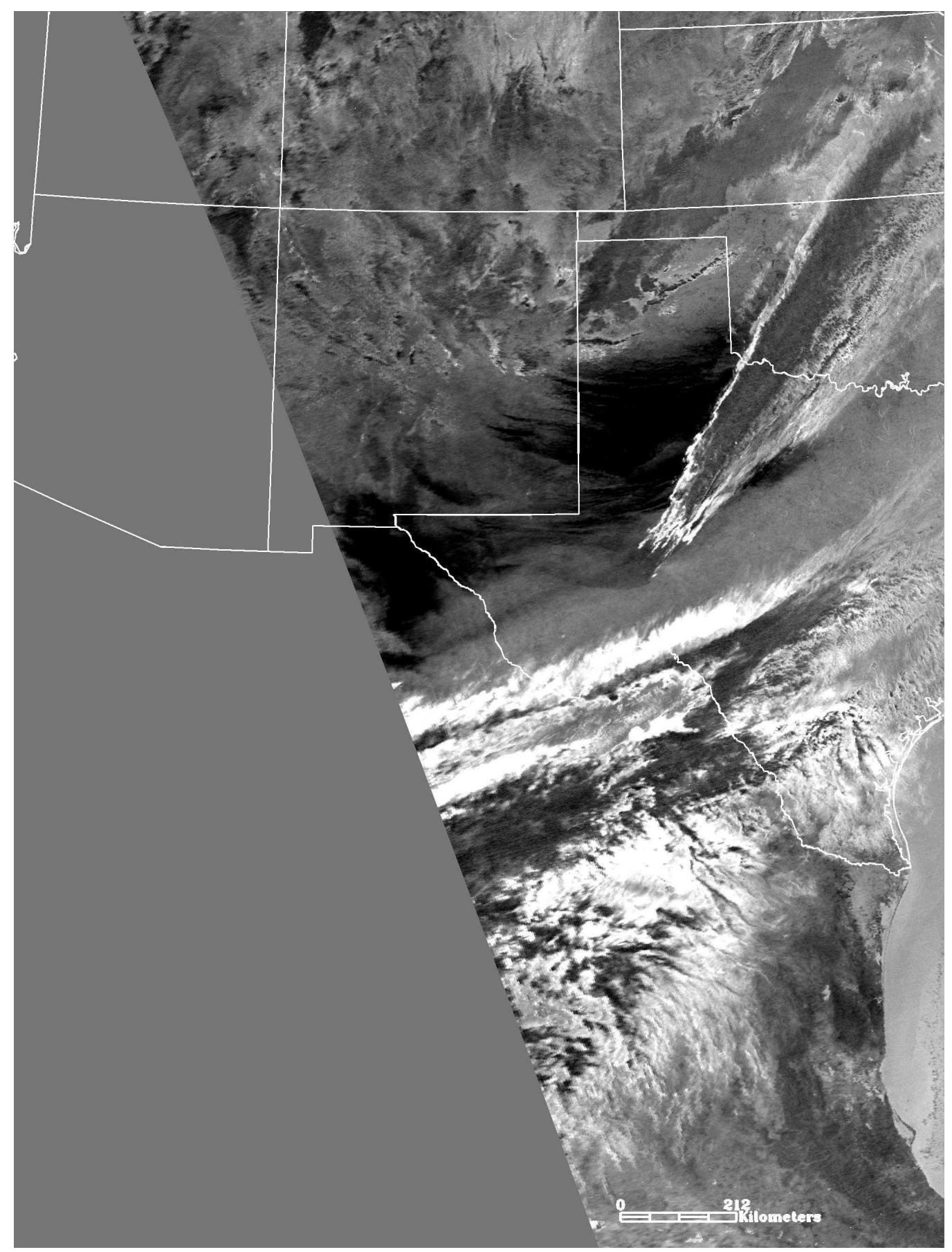

Figure 2.13: MNF Component 5 of December 15, 2003 event 


\subsection{4 k-means Classification}

Pattern classification involves extraction of features from the image and using these features to classify the images. It is used as the post-processing step to feature extraction and analysis. Classification mainly deals with labeling an image into classes where a single class represents many pixels with the same feature. Various comparison methods are used to determine if a specific pixel belongs to a class member [21].

Classification algorithms are grouped into two categories :

- Supervised Classification (learning with teacher) a set of training samples are available for teaching the computer how to recognize patterns;

- Unsupervised Classification (learning without a teacher) no such a priori knowledge of training samples is available. The computer has to learn by itself.

In remote sensing, classification methods have been commonly used for the mapping of minerals, land and vegetative cover of multi spectral data sets. Typical applications of classification are in computer vision, medical imaging, object classification for robot control, speech recognition and image compression [21].

The k-means algorithm is one of the simplest unsupervised classification technique. This technique is used to cluster 'n' objects based on attributes into 'k' partitions, $k<n$. An initial mean vector is specified arbitrarily for each of the $\mathrm{k}$ clusters. Each pixel of the training set is assigned to the class whose mean vector is closest to the pixel vector. Then, a new set of cluster mean vectors are calculated. Based on these values, the pixels are reassigned. For each iteration, $\mathrm{k}$ means will move towards the data concentration in their current region of feature space. This process is continued until there is no significant change in the pixel assignments from the previous assignment. 
Figure 2.14 shows a hypothetical illustration of the k-means clustering algorithm [34]. The first block shows the initial randomized point and a number of points available for clustering. The second step indicates the points that are associated with the nearest initial randomized point. The initial randomized points are moved to the center of their respective clusters or the centroids in the step 3. These steps 2 and 3 are repeated until a suitable level of convergence has been reached. The final block shows the final result of k-means clustering.

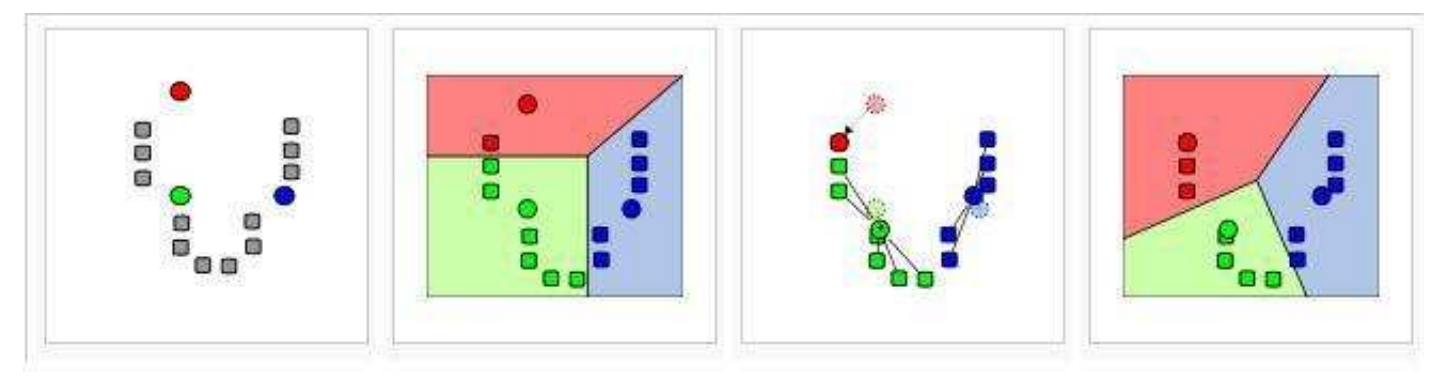

Figure 2.14: Step-by-step k-means Clustering Algorithm [35]

Mathematical background :

Suppose $\vec{X}=\left[x_{1}, x_{2}, \ldots, x_{N}\right]$ is a data set consisting of $\mathrm{N}$ observations of a random D-dimensional vector $\mathrm{x}$ [25]. The goal of k-means clustering is to partition the data set into $\mathrm{k}$ number of clusters assuming an initial value for $\mathrm{k}$.

Consider a set of D-dimensional initial mean vectors $\mu_{1}, \mu_{2}, \ldots . \mu_{k}$. The data points are assigned to k clusters and the set of vectors $\mu_{k}$, such that the sum of squares of distances of each data point to its closest vector $\mu_{k}$ is minimum.

For each data point $x_{n}$, we introduce a corresponding set of binary indicator variables $r_{n k} \in 0,1$, where $\mathrm{k}=1,2, \ldots, \mathrm{K}$ describing which of the $\mathrm{K}$ clusters the data point $x_{n}$, is assigned to. If data point $x_{n}$ is assigned to cluster $\mathrm{k}$ then $r_{n k}=1$ and $r_{n j}$ $=0$ for $j \neq k$. This is also called a 1 -of-K coding scheme. 
The distortion measure is given by

$$
J=\sum_{n=1}^{N} \sum_{k=1}^{K} r_{n k}\left\|\left(x_{n}-\mu_{k}\right)\right\|^{2},
$$

which represents the sum of squares of the distances of each data point to its assigned vector $\mu_{k}$.

The objective is to find the values for $r_{n k}$ and the $\mu_{k}$ to minimize J. Now, for each cluster, the inter-point distances are small compared with the distances outside the cluster.

Thus, an algorithm for k-Means Classfication proceeds as follows [20] :

1. begin initialize $\mathrm{n}, \mathrm{c}, \mu_{1}, \mu_{2} \ldots ., \mu_{k}$

2. do classify $\mathrm{k}$ samples according to nearest $\mu_{i}$

3. recompute $\mu_{i}$

4. until no change in $\mu_{i}$

5. return $\mu_{1}, \mu_{2}, \ldots . ., \mu_{k}$

6. end

The computational complexity of the algorithm is $O(n \times d \times c \times T)$ where, $\mathrm{n}$ is the known number of patterns, $\mathrm{c}$ is the desired number of clusters, $\mathrm{d}$ is the number of features and $\mathrm{T}$ is the number of iterations. Usually, $T<d$.

The net mean migration is defined as the magnitude change of mean vectors from iteration i-1 to iteration i over k clusters [15].

$$
\triangle \mu(i)=\sum_{k=1}^{K}\left|\left(\mu_{k}^{i}-\mu_{k}^{i-1}\right)\right|
$$

The drawback of $\mathrm{k}$-means algorithm is that it is very sensitive to initial values. An inappropriate choice for number of clusters ' $k$ ' results in poor classification results. 


\section{Chapter 3}

\section{Visualization technique for dust storm detection}

\subsection{Block Diagram}

For visual interpretation and locating the origin of a dust storm, directional filtering is applied on the cropped individual image bands at different angles. Energy and entropy measurements are computed to find the prominent direction of the dust storm. Edge detectors like Sobel and Frei-Chen are applied to the selected filtered images for further enhancement of the streaks produced by the directional texture. False color composite images are created to differentiate the dust storm image texture and mark the origin of the resultant directional streaks.

Figure 3.1 provides an overview of the visualization technique. In addition, we show some of the cropped original images in Figures 3.2 and 3.3 in order to visually compare to the results shown later in this chapter. From the dust storm images, we can see that a prominent direction applies locally in the dust storm region and the curvature of the dust storm would be an issue in accurately predicting the direction 
of the dust storm.

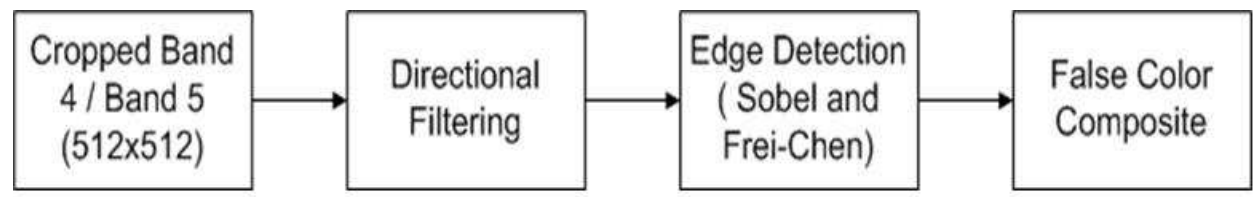

Figure 3.1: Block diagram for Visualization

\subsection{Directional Filtering}

Directional filters are significant in many image processing applications such as edge sharpening, feature enhancement, texture synthesis, object recognition.

In this research, we use two types of directional filters - Steerable Gaussian filters and Directional filters available in ENVI.

Steerable Gaussian filters are designed to apply the same filter, rotated at different angles adaptively and calculate the filter response at these angles. Steerable filters is a class of filters in which a filter of arbitrary orientation is synthesized as a linear combination of a set of basis filters [14].

Consider the two-dimensional circularly symmetric Gaussian function $G(x, y)=$ $e^{-\left(x^{2}+y^{2}\right)}$. Let the $n$th derivative of a Gaussian in the $x$ direction as $G_{n}$. Let $f^{\theta}(x, y)$ represent $\mathrm{f}(\mathrm{x}, \mathrm{y})$ rotated through an angle $\theta$ about the origin.

The first $x$ derivative of a Gaussian

$$
G_{1}^{0^{\circ}}=\frac{\partial}{\partial x}\left[e^{-\left(x^{2}+y^{2}\right)}\right]=-2 x e^{-\left(x^{2}+y^{2}\right)}
$$

If rotated by $90^{\circ}$,

$$
G_{1}^{90^{\circ}}=\frac{\partial}{\partial y}\left[e^{-\left(x^{2}+y^{2}\right)}\right]=-2 y e^{-\left(x^{2}+y^{2}\right)}
$$




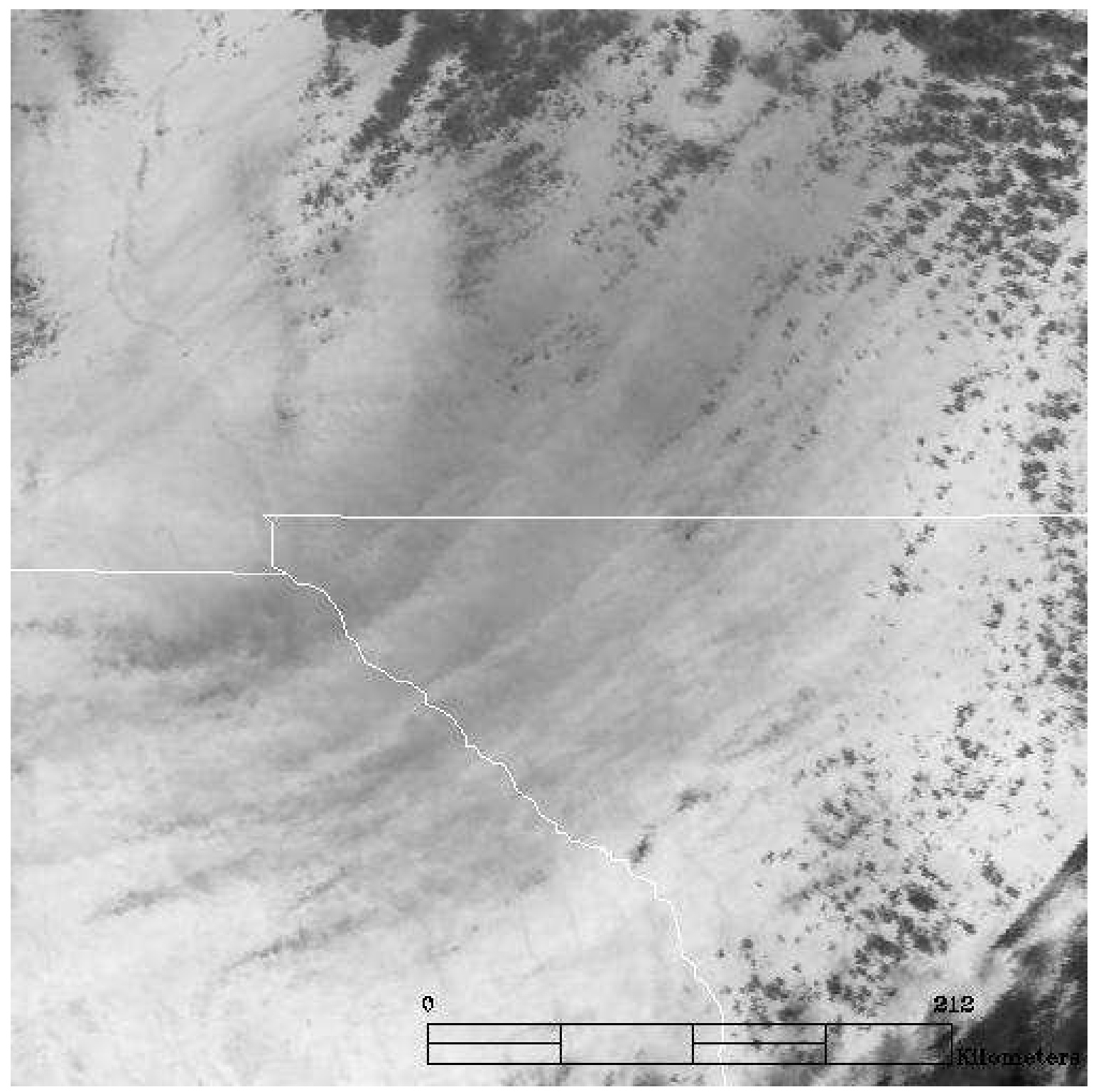

Figure 3.2: Cropped 512x512 band 4 of April 15, 2003 event 


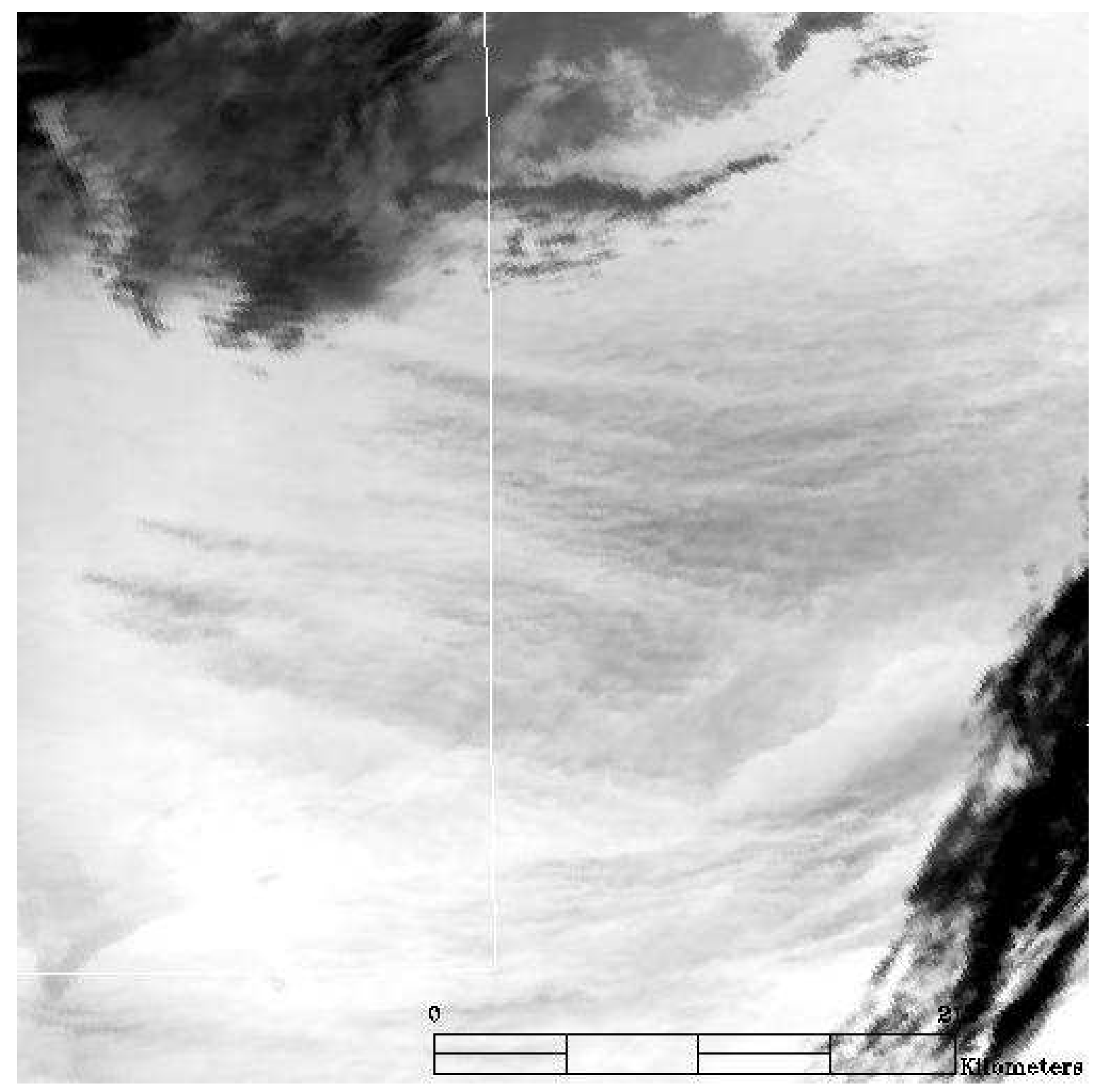

Figure 3.3: Cropped 512x512 band 4 of December 15, 2003 event 
Thus, the filter $G_{1}$ can be at any arbitrary orientation $\theta$ can be synthesized by a linear combination

$$
G_{1}^{\theta}=\cos (\theta) G_{1}^{0^{\circ}}+\sin (\theta) G_{1}^{90^{\circ}}
$$

$G_{1}^{0^{\circ}}$ and $G_{1}^{90^{\circ}}$ are called the basis filters for $G_{1}^{\theta} \cdot \cos (\theta)$ and $\sin (\theta)$ are the interpolation functions for the basis filters.

We can synthesize the image filtered at an arbitrary orientation using convolution by taking linear combination of images filtered with $G_{1}^{0^{\circ}}$ and $G_{1}^{90^{\circ}}$

Therefore,

$$
\begin{gathered}
R_{1}^{0^{\circ}}=G_{1}^{0^{\circ}} * I \\
R_{1}^{90^{\circ}}=G_{1}^{90^{\circ}} * I \\
R_{1}^{\theta}=\cos (\theta) R_{1}^{0^{\circ}}+\sin (\theta) R_{1}^{90^{\circ}}
\end{gathered}
$$

Figure 3.4 shows the results of directional filtering at different angles.

ENVI Directional Filters are one of the standard convolution filters. Convolution filters results in images which a certain pixel value is the function of some weighted average of the brightness of the neighboring pixels. The sum of the directional filter kernel elements is zero. Convolution of this filter mask with the image results in the spatially filtered image. Directional filter is a first derivative edge enhancement filter that selectively enhances image features having specific direction components (gradients). It performs spatial high pass filtering. The resultant image after convolution with the directional filter mask contains areas with uniform pixel values represented by 0 and those with variable values are presented as bright edges. A 3x3 ENVI directional filter mask at 80 degrees is shown in Table 3.1. Figure 3.5 shows the result of ENVI directional filtering at 80 degrees. 

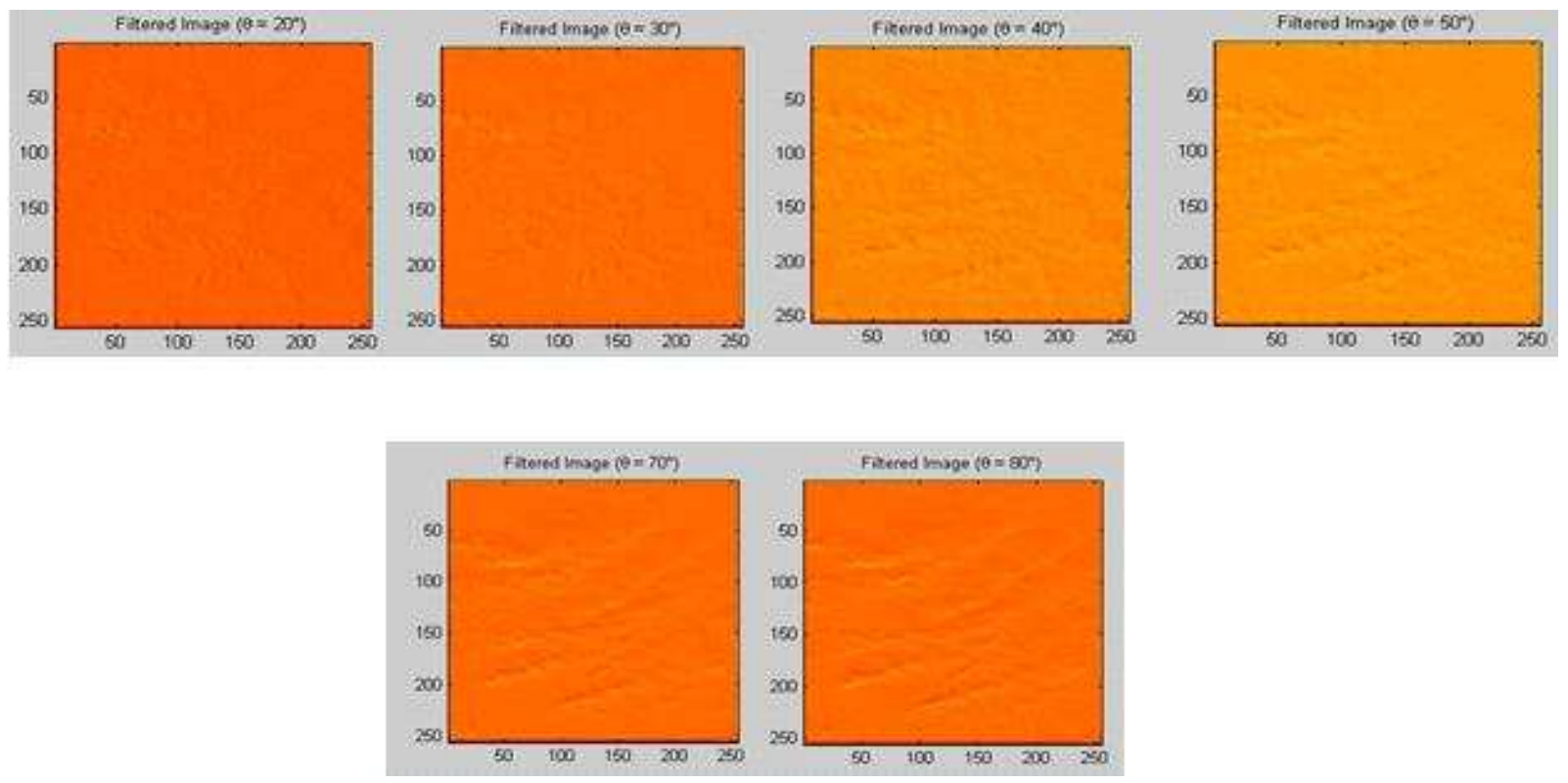

Figure 3.4: Directional filtering of cropped band 4 for different angles $20^{\circ}$, $30^{\circ}, 40^{\circ}, 50^{\circ}, 70^{\circ}, 90^{\circ}$ (from left to right)

Table 3.1: A 3x3 ENVI directional filter mask at 80 degrees

\begin{tabular}{|c|c|c|}
\hline-1.1585 & -0.9848 & -0.8112 \\
\hline-0.1736 & 0 & 0.1736 \\
\hline 0.8112 & 0.9848 & 1.1585 \\
\hline
\end{tabular}




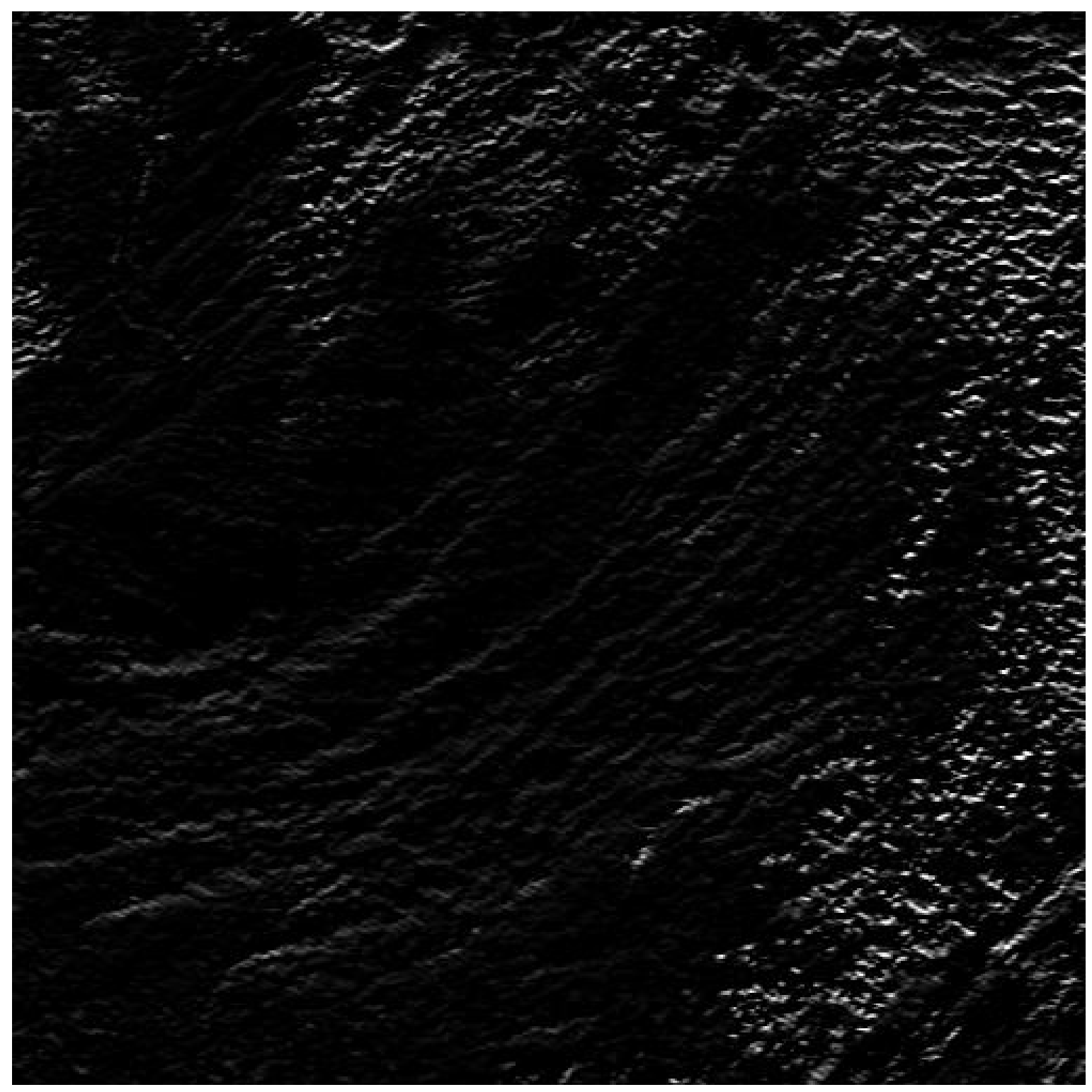

Figure 3.5: Directional filtering of cropped band 4 at 80 degrees for April 15, 2003 event 


\subsection{Edge Detection}

Edge detection is very important local image pre-processing method used to process images to generate an image with sharper edges and improved interpretability. It is mainly used for feature detection and extraction. Edge detectors are used locate changes in the intensity function. The detection of significant pixel changes from one pixel to another is a common problem in image processing [15].

A large change in the image brightness over a short spatial distance indicates the presence of an edge. An edge is a property attached to an individual pixel and is calculated from the image function behavior in a neighborhood of that pixel [16]. It is a vector variable with two components - magnitude and direction.

The directional high pass filters like Roberts, Sobel and Prewitt are based on the directional gradient operators i.e., they produce images whose DN values are proportional to the difference between neighboring pixel DNs in the given direction. The magnitude of the local image gradient is given by the length of the composite vector and the coordinate axis (see figure below) [15].

At each image point, the gradient vector points in the direction of largest possible intensity increase, and the length of the gradient vector corresponds to the rate of change in that direction.

The gradient image is obtained by subtracting the gray-level value of a neighboring pixel. If the neighboring pixel is chosen from the same image row, a directional derivative in the $\mathrm{X}$ direction is obtained; if the neighboring pixel is chosen from the same image column, a directional directive in the $\mathrm{Y}$ direction results and can be represented as $\mathrm{dG} / \mathrm{dy}$. The isotropic first derivative, or gradient, is computed as the square root of the sum of the squares of those two directional derivatives:

$$
\left.\operatorname{grad} G=\left[(d G / d x)^{2}+(d G / d y)^{2}\right)\right]
$$




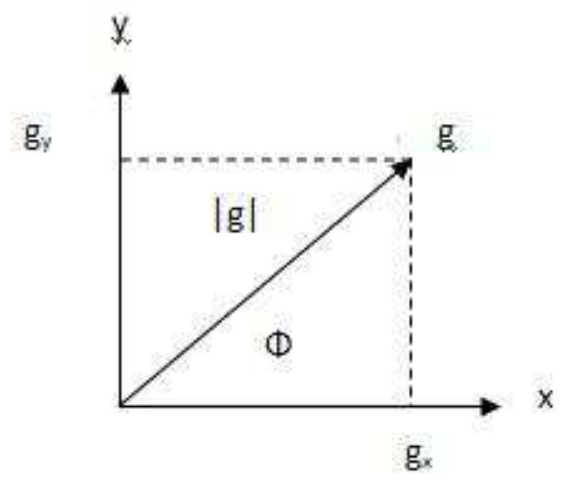

\section{Figure 3.6: Calculating Image Gradients}

High values for grad $\mathrm{G}$ indicate the presence of edges or abrupt changes in graylevel value.

The gradient of the function $\mathrm{f}(\mathrm{x}, \mathrm{y})$ is defined as the two-dimensional column vector,

$$
\nabla f=\left[\begin{array}{l}
G_{x} \\
G_{y}
\end{array}\right]=\left[\begin{array}{l}
\partial f / \partial x \\
\partial f / \partial y
\end{array}\right]
$$

The magnitude of this vector is given by

$$
\begin{gathered}
\nabla=\operatorname{mag}(\nabla f) \\
=\sqrt{\left[G_{x}^{2}+G_{y}^{2}\right]} \\
=\sqrt{\left[(\partial f / \partial x)^{2}+(\partial f / \partial y)^{2}\right]} \\
\approx\left|G_{x}\right|+\left|G_{y}\right|
\end{gathered}
$$

The direction of the vector $\nabla f$ at $(\mathrm{x}, \mathrm{y})$ is given by

$$
\alpha(x, y)=\arctan \left(G_{y} / G_{x}\right)
$$


where the angle is measured with respect to $\mathrm{x}$-axis. The direction of an edge is perpendicular to the direction of the gradient vector at that point.

A 3x3 area of an image with z's as gray-level values used to compute gradient at point $z_{5}$ can be represented as

Table 3.2: A 3x3 region of an image representing gray-level values

\begin{tabular}{|l|l|l|}
\hline$z_{1}$ & $z_{2}$ & $z_{3}$ \\
\hline$z_{4}$ & $z_{5}$ & $z_{6}$ \\
\hline$z_{7}$ & $z_{8}$ & $z_{9}$ \\
\hline
\end{tabular}

\subsubsection{Sobel Edge Detector}

Sobel operator is a simple edge detector. The Sobel operator approximates the gradient by using the row and column mask to approximate the first derivative in each direction. The Sobel edge detection masks look for edges in both the horizontal and vertical directions, and then combine this information. The horizontal and vertical sobel masks are as shown below.

Table 3.3: Horizontal Sobel Operator

\begin{tabular}{|l|l|r|}
\hline-1 & -2 & -1 \\
\hline 0 & 0 & 0 \\
\hline 1 & 2 & 1 \\
\hline
\end{tabular}

Each of these $3 \times 3$ masks are convolved with the image. So, each pixel has two edge magnitudes and directions corresponding to the horizontal and vertical directions [17]. 
Table 3.4: Vertical Sobel Operator

\begin{tabular}{|l|l|l|}
\hline-1 & 0 & 1 \\
\hline-2 & 0 & 2 \\
\hline-1 & 0 & 1 \\
\hline
\end{tabular}

The gradient at point labeled $z_{5}$ is computed as

$$
\begin{aligned}
& G_{x}=\left(z_{7}+2 z_{8}+z_{9}\right)-\left(z_{1}+2 z_{2}+z_{3}\right) \\
& G_{y}=\left(z_{3}+2 z_{6}+z_{9}\right)-\left(z_{1}+2 z_{4}+z_{7}\right)
\end{aligned}
$$

A weight value of 2 is used to achieve some smoothing.

Edge magnitude is given by $\sqrt{G_{x}^{2}+G_{y}^{2}}$

Edge direction is given by $\arctan \left[G_{x} / G_{y}\right]$

The edge direction is perpendicular to the line, because the specified direction is the direction of the gradient along which the gray levels are changing [16].

Figure 3.7 shows the result of sobel edge detection on ENVI directional filtered image at 80 degrees for the April 15, 2003 event.

\subsubsection{Frei-Chen Edge Detector}

Frei-Chen is one of the advanced edge detector algorithm [17]. It can be used to find edges and lines of specific orientation.

Edge detection using the Frei-Chen masks is implemented by mapping the intensity vector using a linear transformation and then detecting edges based on the angle between intensity vector and it's projection onto the edge subspace. The basis vectors are the Frei-Chen masks and the weights are the projection values. Any 3x3 subimage can be represented as the weighted sum of the basis vectors. The weights 


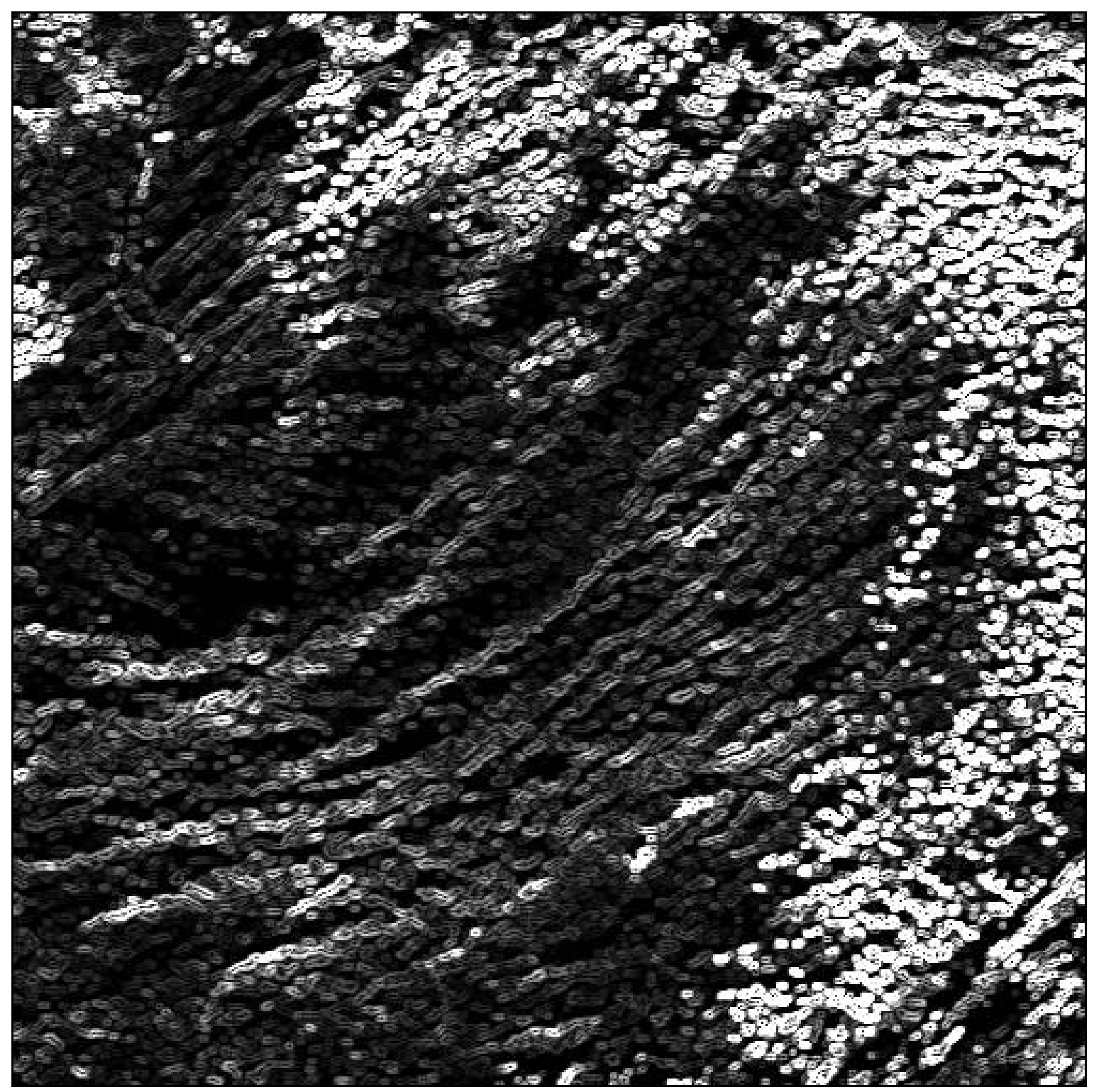

Figure 3.7: Sobel Edge Detection on ENVI directional filtered image of April 15, 2003 at 80 degrees 
are found by projecting the subimage onto each basis vector i.e., performing a vector inner product. The following are the 3x3 Frei-Chen masks used for edge detection.

Table 3.5: Horizontal Frei-Chen Operator

\begin{tabular}{|l|l|r|}
\hline-1 & 0 & +1 \\
\hline$-\sqrt{2}$ & 0 & $+\sqrt{2}$ \\
\hline-1 & 0 & +1 \\
\hline
\end{tabular}

Table 3.6: Vertical Frei-Chen Operator

\begin{tabular}{|l|c|r|}
\hline+1 & $+\sqrt{2}$ & +1 \\
\hline 0 & 0 & 0 \\
\hline-1 & $-\sqrt{2}$ & -1 \\
\hline
\end{tabular}

A particular subspace of interest is selected and the relative projection of the image onto it is found [17]. This is given by the equation :

$$
\cos (\Theta)=\sqrt{M / S}
$$

where

$$
M=\sum_{k \in\{e\}}\left(I_{s}, f_{k}\right)^{2}
$$

and

$$
S=\sum_{k=1}^{9}\left(I_{s}, f_{k}\right)^{2}
$$

The set $\{e\}$ consists of the masks of interest. $\left(I_{s}, f_{k}\right)$ refers to the process of overlaying the mask on the subimage and finding the vector inner product. The 
advantage of this method is that we can select particular edge or line masks of interest, and consider the projection of those masks only.

Figure 3.8 shows the result of Frei-Chen edge detection on ENVI directional filtered image at 80 degrees for April 15, 2003.

\subsection{Locating dust sources using False Color Com- posite images}

After directional filtering at different angles and determining the potential angle for dust storm, edge detection (either Sobel or Frei-Chen method) is applied to the directionally filtered images. False Color Composite is an artificial representation of multispectral image. The display color assignment for any band of a multispectral image is done in an arbitrary manner. Many environmental features are more readily discernible when satellite images are processed as false color composites. False Color Composite images are created using different band combinations to enhance the directional streaks and locate the origin point of dust storms. Figure 3.9 3.10 3.11 show the manually located origin points of dust storm on the false color composite images for the April 15th, 2003 event. RGB band combinations are shown. False Color Composites of different band combinations are show in the figures below. Band combination $1,4,5(\mathrm{R}, \mathrm{G}, \mathrm{B})$ is recommended to locate the dust sources easily by visual interpretation.

The dust storm source locations are pointed out more precisely in the tables 3.7 and 3.8. For April 15, 2003 event 8 out of the 20 stated in Nancy's thesis match with our results [1], and for December 15, 2003 event. the located 13 sources in the table below coincide with 13 of the 145 sources presented in Lee et.al [2](sources are numbered from top to bottom). 


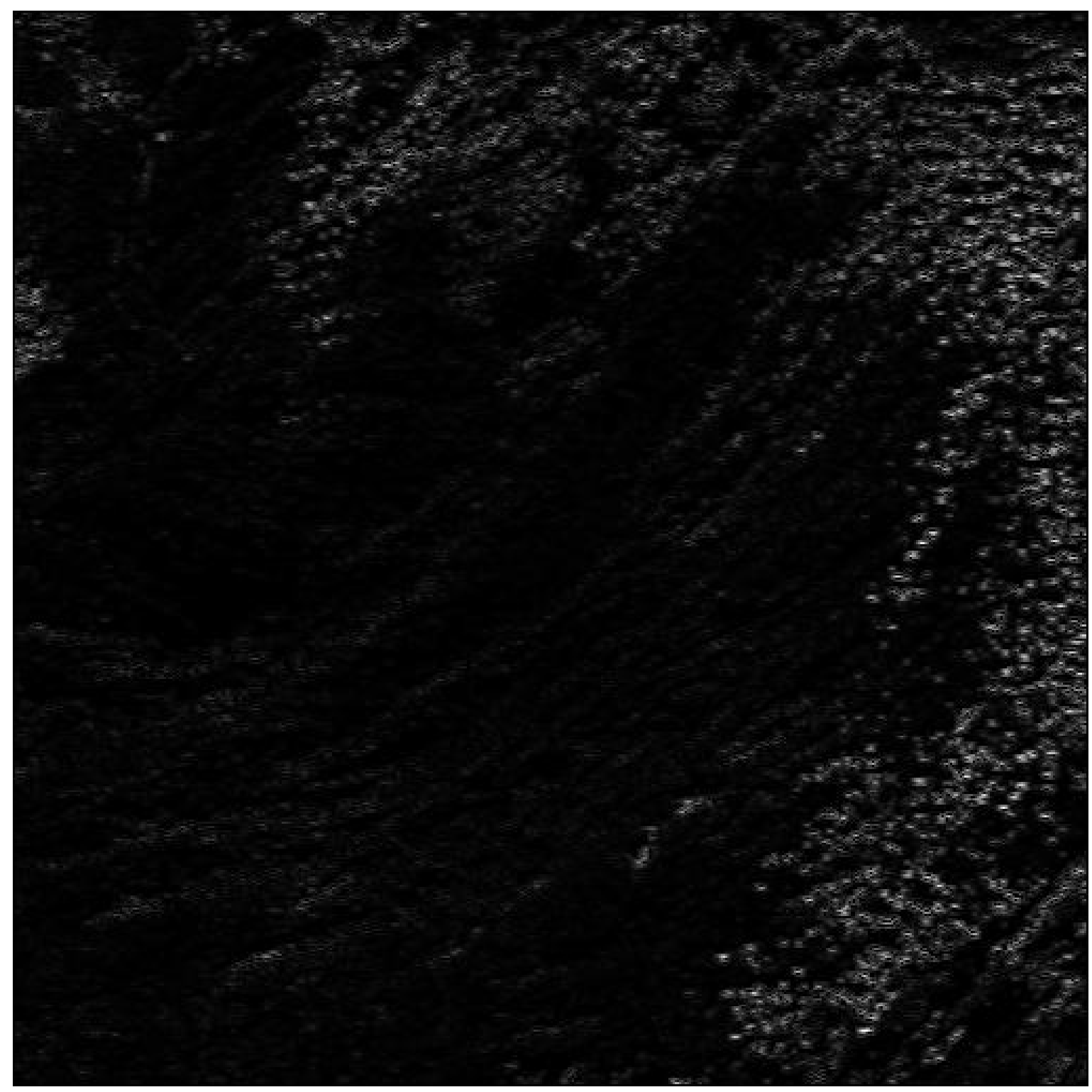

Figure 3.8: Frei-Chen Edge Detection on ENVI directional filtered image of April 15, 2003 at 80 degrees 


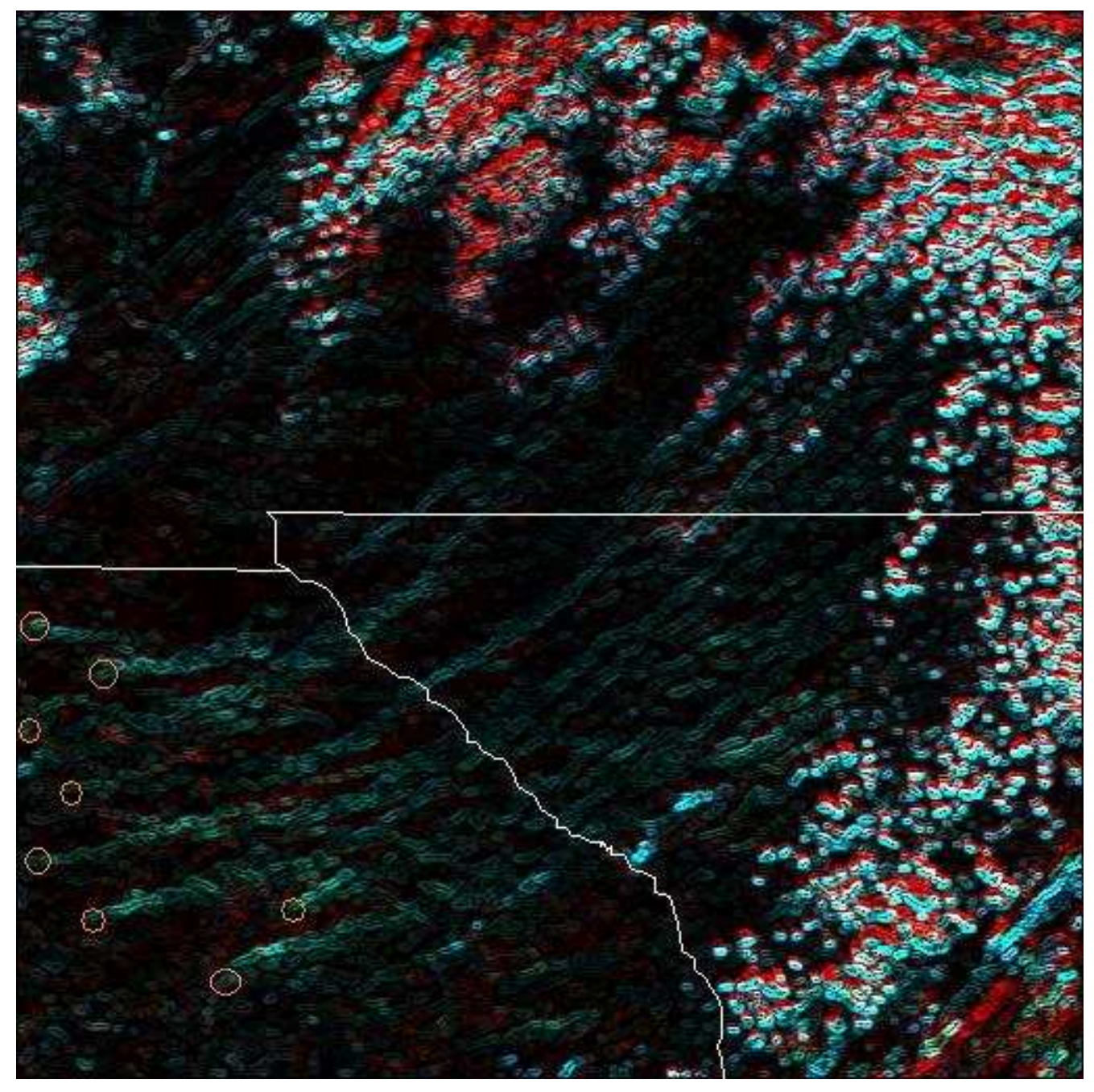

Figure 3.9: False Color Composite image - Band 1, 4, 5 (R, G, B) with marked dust sources for April 15, 2003 event 


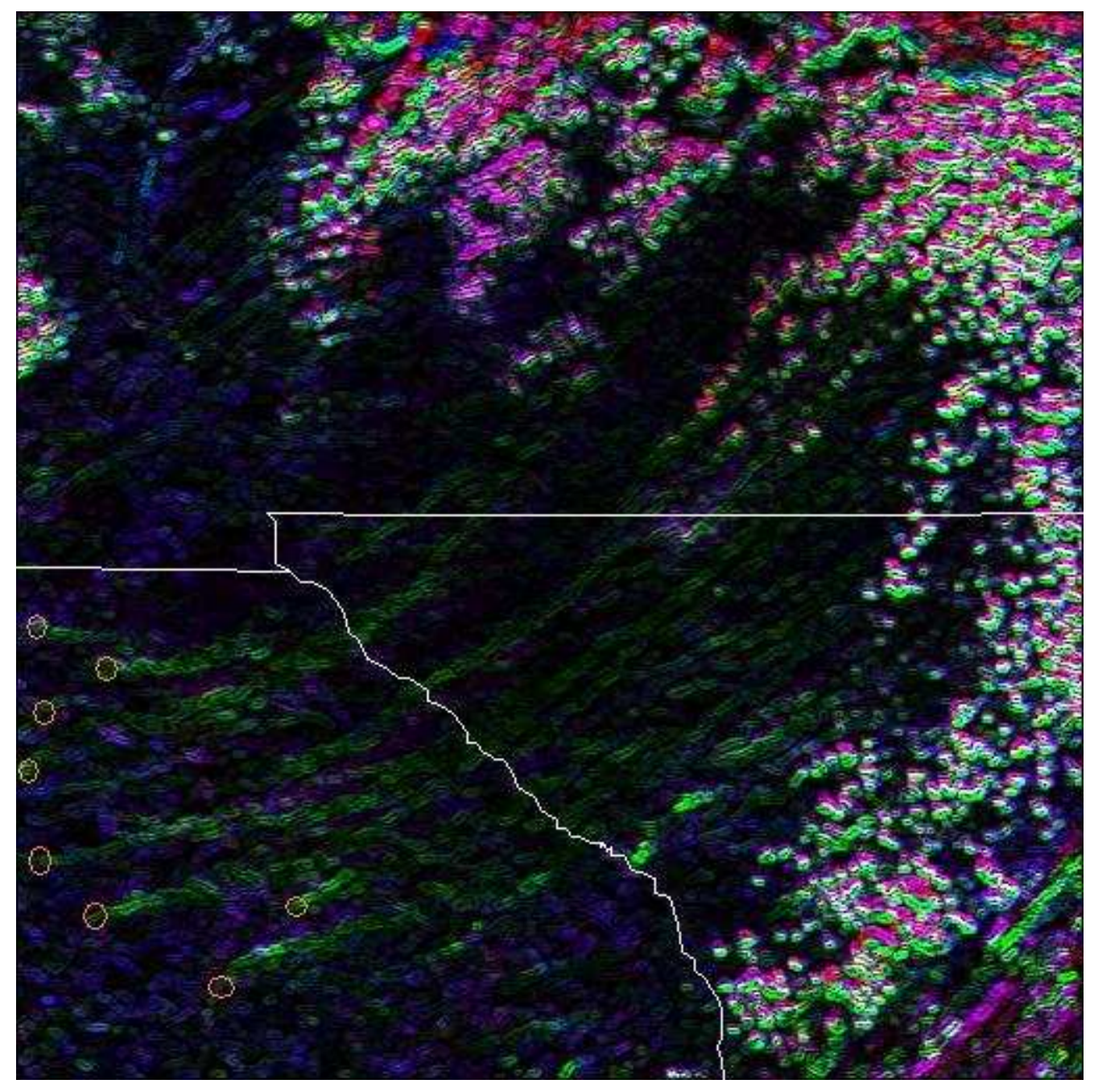

Figure 3.10: False Color Composite image - Band 1, 4, 3 (R, G, B) with marked dust sources for April 15, 2003 event 


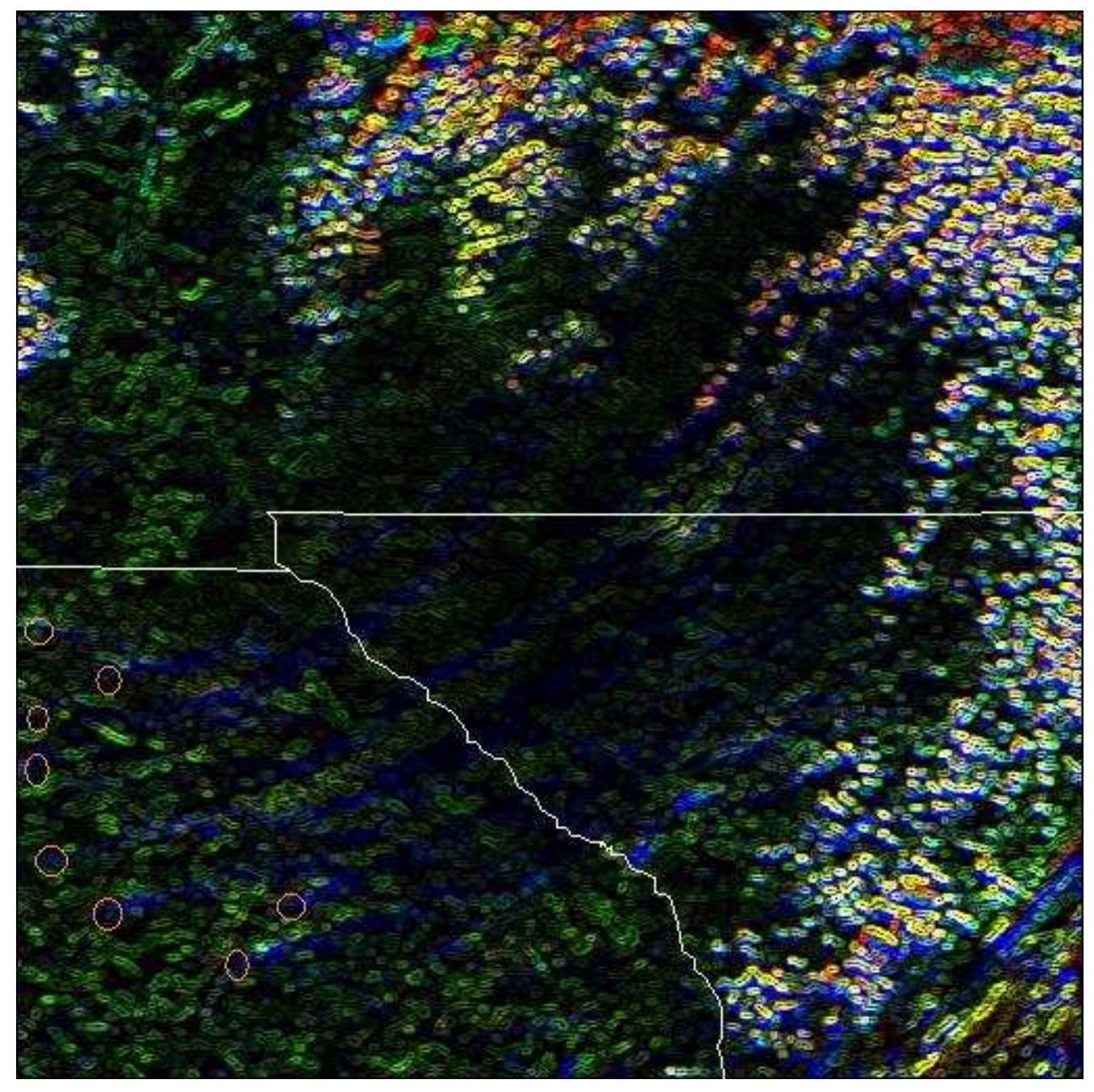

Figure 3.11: False Color Composite image - Band 1, 3, 4 (R, G, B) with marked dust sources for April 15, 2003 event 


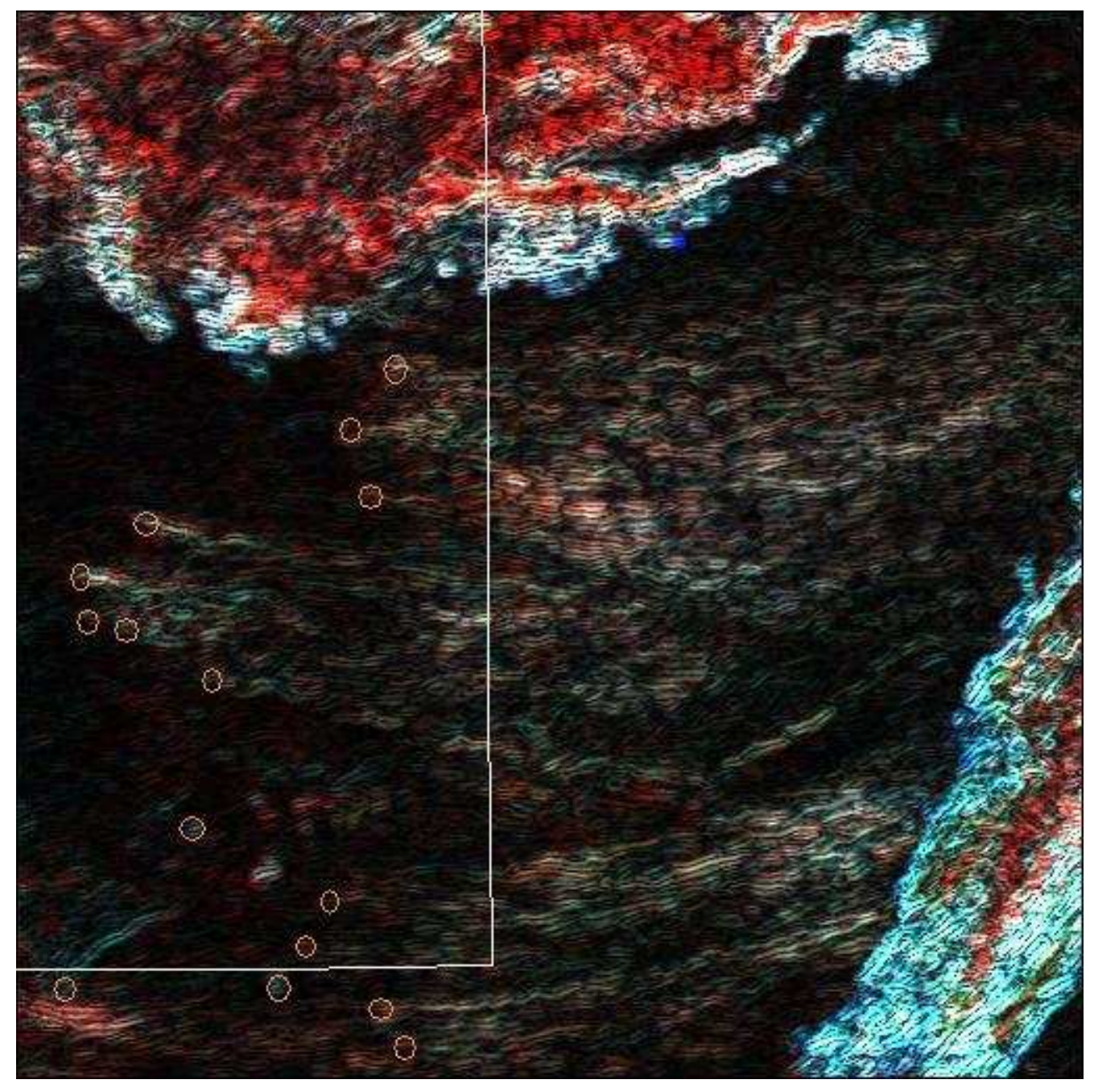

Figure 3.12: False Color Composite image - Band 1, 4, 5 (R, G, B) with marked dust sources for December 15, 2003 event 
Table 3.7: Dust storm source locations (latitude, longitudes) from NOAA-16 satellite image for April 15, 2003 20:23 UTC event

\begin{tabular}{|c|c|c|}
\hline Source & Latitude (deg.N) & Longitude(deg. W) \\
\hline 1 & $31^{\circ} 34^{\prime} 33.59^{\prime \prime}$ & $-107^{\circ} 37^{\prime} 39.53^{\prime \prime}$ \\
\hline 2 & $31^{\circ} 11^{\prime} 59.04^{\prime \prime}$ & $-107^{\circ} 40^{\prime} 47.68^{\prime \prime}$ \\
\hline 3 & $31^{\circ} 2^{\prime} 48.02^{\prime \prime}$ & $-107^{\circ} 40^{\prime} 32.67^{\prime \prime}$ \\
\hline 4 & $30^{\circ} 58^{\prime} 26.20^{\prime \prime}$ & $-107^{\circ} 28^{\prime} 41.91^{\prime \prime}$ \\
\hline 5 & $30^{\circ} 43^{\prime} 36.00^{\prime \prime}$ & $-107^{\circ} 35^{\prime} 46.19^{\prime \prime}$ \\
\hline 6 & $30^{\circ} 31^{\prime} 0.13^{\prime \prime}$ & $-107^{\circ} 21^{\prime} 38.63^{\prime \prime}$ \\
\hline 7 & $30^{\circ} 33^{\prime} 34.84^{\prime \prime}$ & $-106^{\circ} 31^{\prime} 16.96^{\prime \prime}$ \\
\hline 8 & $30^{\circ} 16^{\prime} 22.06^{\prime \prime}$ & $-106^{\circ} 48^{\prime} 28.94^{\prime \prime}$ \\
\hline
\end{tabular}


Table 3.8: Dust storm source locations (latitude, longitudes) from NOAA-16 satellite image for December 15, 2003 19:51 UTC event

\begin{tabular}{|c|c|c|}
\hline Source & Latitude (deg.N) & Longitude(deg.W) \\
\hline 1 & $34^{\circ} 14^{\prime} 12.66^{\prime \prime}$ & $-103^{\circ} 29^{\prime} 3.23^{\prime \prime}$ \\
\hline 2 & $33^{\circ} 56^{\prime} 23.15^{\prime \prime}$ & $-103^{\circ} 40^{\prime} 52.56^{\prime \prime}$ \\
\hline 3 & $33^{\circ} 45^{\prime} 16.75^{\prime \prime}$ & $-103^{\circ} 34^{\prime} 26.85^{\prime \prime}$ \\
\hline 4 & $33^{\circ} 40^{\prime} 37.70^{\prime \prime}$ & $-104^{\circ} 34^{\prime} 47.70^{\prime \prime}$ \\
\hline 5 & $33^{\circ} 27^{\prime} 19.38^{\prime \prime}$ & $-104^{\circ} 49^{\prime} 36.85^{\prime \prime}$ \\
\hline 6 & $33^{\circ} 18^{\prime} 35.08^{\prime \prime}$ & $-104^{\circ} 48^{\prime} 32.29^{\prime \prime}$ \\
\hline 7 & $33^{\circ} 13^{\prime} 29.01^{\prime \prime}$ & $-104^{\circ} 31^{\prime} 5.74^{\prime \prime}$ \\
\hline 8 & $33^{\circ} 4^{\prime} 40.79^{\prime \prime}$ & $-104^{\circ} 15^{\prime} 53.50^{\prime \prime}$ \\
\hline 9 & $32^{\circ} 20^{\prime} 50.10^{\prime \prime}$ & $-103^{\circ} 9^{\prime} 12.11^{\prime \prime}$ \\
\hline 10 & $32^{\circ} 9^{\prime} 3.45^{\prime \prime}$ & $-103^{\circ} 22^{\prime} 22.31^{\prime \prime}$ \\
\hline 11 & $32^{\circ} 3^{\prime} 24.50^{\prime \prime}$ & $-103^{\circ} 54^{\prime} 15.64^{\prime \prime}$ \\
\hline 12 & $31^{\circ} 54^{\prime} 56.87^{\prime \prime}$ & $-104^{\circ} 57^{\prime} 18.66^{\prime \prime}$ \\
\hline 13 & $31^{\circ} 51^{\prime} 34.49^{\prime \prime}$ & $-103^{\circ} 20^{\prime} 30.72^{\prime \prime}$ \\
\hline
\end{tabular}




\section{Chapter 4}

\section{Techniques for automating the detection of dust storms}

\subsection{Block Diagram}

In the proposed automated dust storm detection technique, we perform block processing on the cropped $512 \times 512$ region of band 4 and band 5 images. Block processing means partitioning an image into blocks and processing them individually and parallely. The optimal block size chosen in our analysis is $128 \times 128$. This is based on the limited resolution of resultant images from the power spectrum analysis. The processing steps are performed on these 16 blocks of each 128x128 size. A local power spectral density analysis is first used to confirm the presence of high directionality information in certain regions of an image. These regions can be selected as candidate dust storm regions. The presence of a prominent direction in the texture of the candidate dust storm region can be used as a verification step in this automated detection scheme. Binary Thresholding based on Otsu's automatic thresholding method is performed on these blocks to enhance the directional textures. Morphological enhancement is 


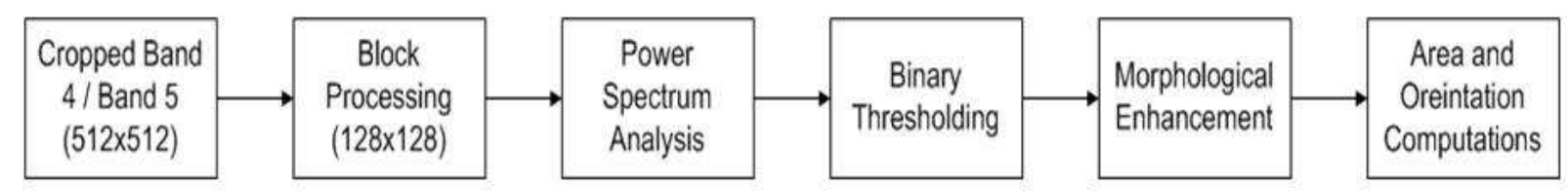

Figure 4.1: Block diagram for Automated dust storm detection

\subsection{Power Spectrum Analysis}

The magnitude squared of the Fourier transform is called the power spectral density (PSD), or power spectrum [27].

The Discrete Fourier Transform (DFT) of an image $\mathrm{f}(\mathrm{x}, \mathrm{y})$ of size $\mathrm{MxN}$ is given by

$$
F(u, v)=\frac{1}{M N} \sum_{x=0}^{M-1} \Sigma_{y=0}^{N-1} f(x, y) e^{-j 2 \pi(u x / M+v y / N)}
$$

The variables $\mathrm{x}, \mathrm{y}$ are spatial variables and $\mathrm{u}, \mathrm{v}$ are the transform or frequency variables.

The Fourier spectrum is given by

$$
|F(u, v)|=\sqrt{R^{2}(x, y)+I^{2}(x, y)}
$$

and

the phase angle is given by

$$
\phi(u, v)=\arctan \left[\frac{I(u, v)}{R(u, v)}\right] .
$$


Therefore, the power spectrum is given by

$$
P(u, v)=|F(u, v)|^{2}=R^{2}(u, v)+I^{2}(u, v)
$$

, where $\mathrm{R}(\mathrm{u}, \mathrm{v})$ and $\mathrm{I}(\mathrm{u}, \mathrm{v})$ are the real and imaginary parts of $\mathrm{F}(\mathrm{u}, \mathrm{v})$ respectively.

The power spectrum has the directional information about linear features (edges, lines) in the image. The highest power components are orthogonal to the direction of such objects in the image [15]. Figure 4.2 shows the example power spectra for $256 \times 256$ aerial photographic images. The power spectrum depends on the image spatial structure. The 'desert' image has no directional features (isotropic). The other images have directional content of various degrees. The power spectrum components are orthogonal to this directional content in the spatial domain.

An example image is of Landsat TM band 4 image of sand dunes and irrigated agriculture near Yuma, Arizona, partitioned into 16 128x128 blocks and the corresponding power spectrum of each block [15]. Figure 4.3 shows the power spectrum of Landsat TM band 4 image partitioned into 128x128 blocks [15].

Applications of Fourier transform also exist in image pattern analysis and recognition [17], [25], [27].

\subsection{Binary Thresholding}

Binary images are the simplest images, whose pixels can take only two values - either 0 or 1 corresponding to black or white respectively. Binary images are often created from gray-scale images via a threshold operation, where every pixel above the threshold values is turned white (1) and those below it are turned black (0). Although, much information is lost in this processing, smaller memory requirements and faster execution times make these techniques advantageous. These types of images are used in computer vision applications for shape and property analysis [17]. 

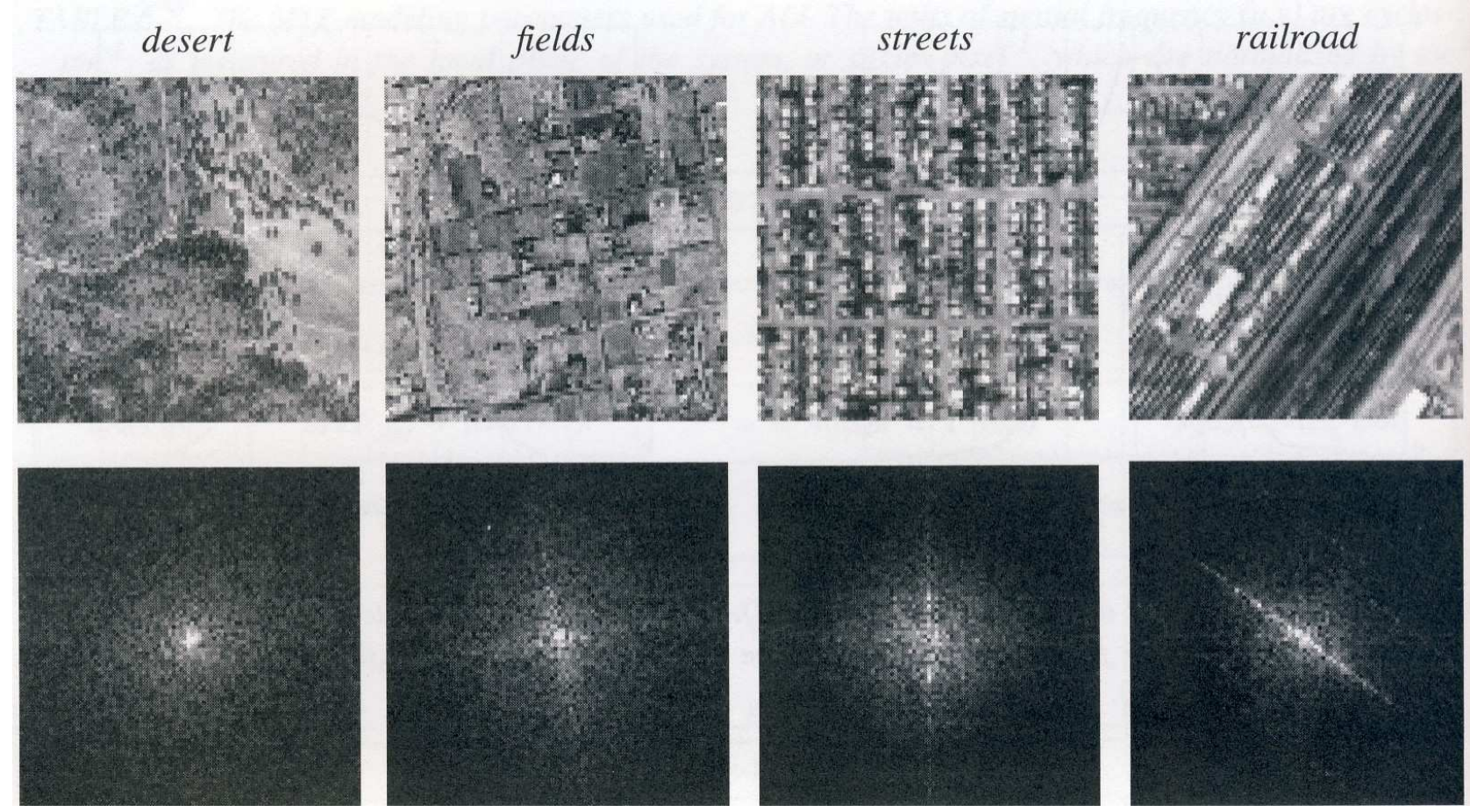

Figure 4.2: Power spectrum analysis for different topographical features [15] 


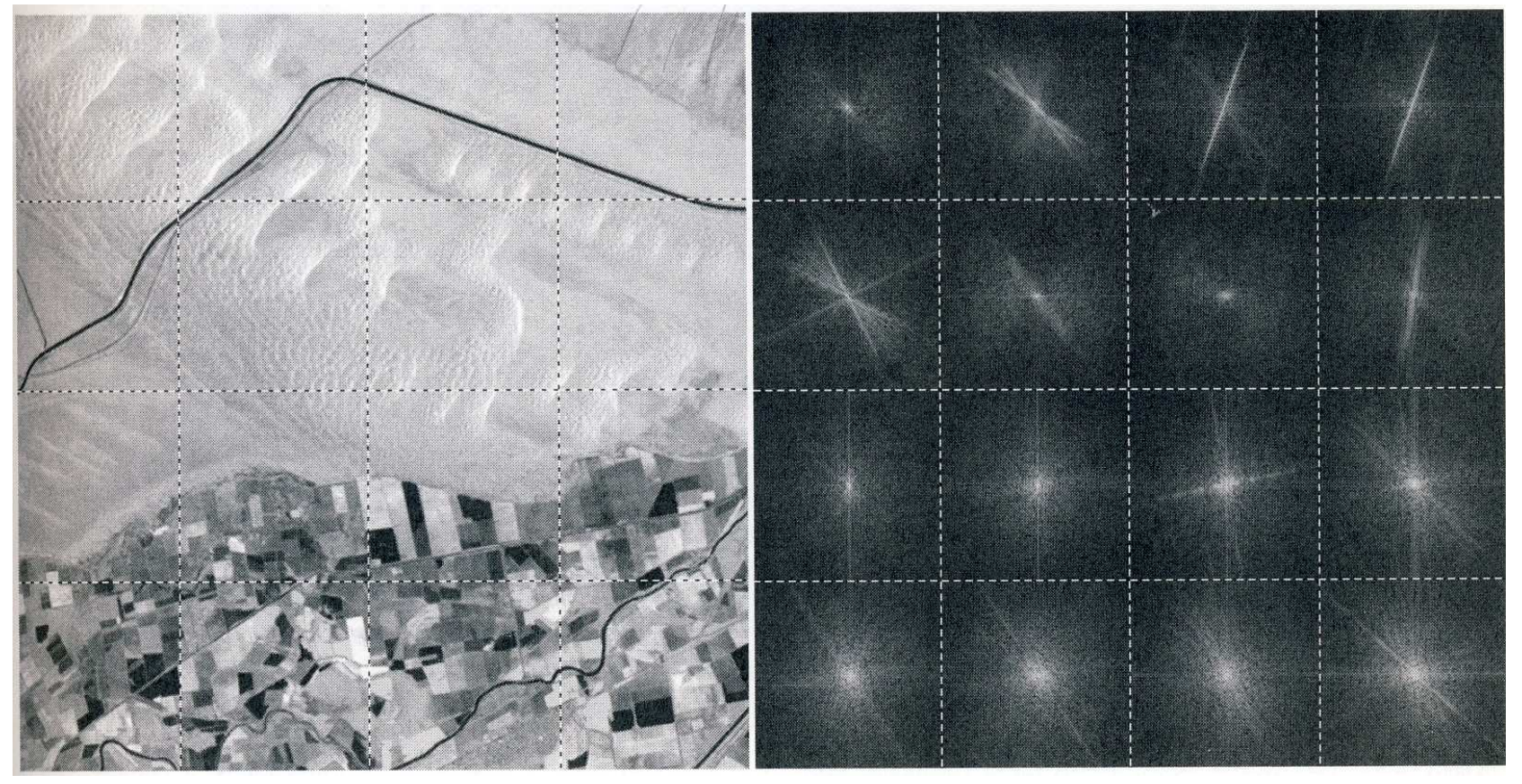

Figure 4.3: Power spectrum analysis by block processing for $128 \times 128$ blocks[15] 
Binary thresholding based on Otsu's automatic thresholding method is applied on the resultant image from the power spectrum analysis [26].

Otsu's method selects the threshold based on the minimization of the within-group variance of the two groups of pixels separated by the thresholding operator. Each threshold ' $t$ ' determines the variance for the group of values that are less than or equal to ' $t$ ' and a variance for the group of values greater than ' $t$ '. The definition for best threshold suggested by Otsu is that threshold for which the weighted sum of within-group variances is minimum. The weights are the probabilities of the respective groups.

Let $\sigma_{w}^{2}(t)$ be the weighted sum of group variances or the within-group variance. Let $\sigma_{1}^{2}(t)$ be the variance for the group with values less than or equal to $\mathrm{t}$ and $\sigma_{2}^{2}(t)$ be the variance for the group with values greater than $t$. Let $q_{1}(t)$ be the probability for the group with values less than or equal to $t$ and $q_{2}(t)$ be the probability for the group with values greater than t. Let $\mu_{1}(t)$ be the mean for the first group and $\mu_{2}(t)$ the mean for the second group. Therefore, the within-group variance $\sigma_{w}^{2}(t)$ is defined by $[13]$

$$
\sigma_{w}^{2}(t)=q_{1}(t) \sigma_{1}^{2}(t)+q_{2}(t) \sigma_{2}^{2}(t),
$$

where $q_{1}(t)=\sum_{i=1}^{t} P(i)$,

$q_{2}(t)=\sum_{i=t+1}^{I} P(i)$,

$\mu_{1}(t)=\sum_{i=1}^{t} i P(i) / q_{1}(t)$,

$\mu_{2}(t)=\sum_{i=t+1}^{I} i P(i) / q_{2}(t)$,

$\sigma_{1}^{2}(t)=\sum_{i=1}^{t}\left[i-\mu_{1}(t)\right]^{2} P(i) / q_{1}(t)$,

$\sigma_{2}^{2}(t)=\sum_{i=t+1}^{I}\left[i-\mu_{2}(t)\right]^{2} P(i) / q_{2}(t)$,

Now, the best possible threshold value ' $t$ ' can be determined by running through full range of 't' values to locate the threshold 't' that minimizes $\sigma_{w}^{2}(t)$. 
The total variance does not depend on threshold value. For any given threshold, the total variance is the sum of the within-class variances and between-class variance. Here, $\sigma^{2}(t)=\sigma_{w}^{2}(t)+\sigma_{B}^{2}(t)$ where the between-class variance $\sigma_{B}^{2}(t)=q_{1}(t)[1-$ $\left.q_{1}(t)\right]\left[\mu_{1}(t)-\mu_{2}(t)\right]^{2}$

Therefore, minimizing the within-class variance is the same as maximizing the between-class variance. The relationship between the within-class variance and betweenclass variances can be exploited to generate a recursion relation that permits a much faster calculation. The quantities in $\sigma_{B}^{2}(t)$ can be computed recursively as we run through the range of ' $t$ ' values.

Initially, $q_{1}(1)=P(1) ; \mu_{1}(0)=0$. By recursion,

$$
\begin{gathered}
q_{1}(t+1)=q_{1}(t)+P(t+1), \\
\mu_{1}(t+1)=\left(q_{1}(t) \mu_{1}(t)+(t+1) P(t+1)\right) / q_{1}(t+1), \\
\mu_{2}(t+1)=\left(\mu-q_{1}(t+1) \mu_{1}(t+1)\right) /\left(1-q_{1}(t+1)\right),
\end{gathered}
$$

Otsu's automatic threshold finder assumes a bimodal distribution of gray-tone values.

\subsection{Morphological Enhancement}

Morphological processing refers to certain operations where an object is hit with a structuring element and thereby reduced to a more revealing shape [25].

Suppose the object $\mathrm{X}$ and the structuring element B are represented as sets in two-dimensional Euclidean space. Let $B_{x}$ denote the translation of $\mathrm{B}$ so that its origin is located at $\mathrm{x}$. The operations of binary morphology input a binary image $\mathrm{B}$ and a structuring element $\mathrm{S}$, which is another, usually much smaller, binary image. The structuring element represents a shape; it can be of any size and have arbitrary 
Table 4.1: A 3x3 structuring element $\mathrm{S}$

\begin{tabular}{|l|l|l|}
\hline 1 & 1 & 1 \\
\hline 1 & 1 & 1 \\
\hline 1 & 1 & 1 \\
\hline
\end{tabular}

structure that can be represented by a binary image. Tables 4.1, 4.2, 4.3 provide an example of closing operation.

The dilation of binary image B by a structuring element $\mathrm{S}$ is denoted by $B \oplus S$ and is defined by

$$
B \oplus S=\cup_{b \epsilon B} S_{b}
$$

The erosion of binary image $\mathrm{B}$ by structuring element $\mathrm{S}$ is denoted by

$$
B \ominus S=b \mid b+s \epsilon B \forall s \epsilon S
$$

The closing of the binary image B by structuring element $\mathrm{S}$ is denoted by

$$
B \bullet S=(B \oplus S) \ominus S
$$

The minimum filter and maximum filter output the local minimum or maximum DN of the input image. If a minimum filter is applied to a binary image of dark and light object, the result - expansion of the dark objects is same as that of a dilation filter, and a maximum filter applied to a binary image is equivalent to an erosion filter. If these two operations are cascaded, an opening or closing operation is obtained.

opening[binary image] $=\operatorname{dilation}[\operatorname{erosion}[$ binary image]

closing[binary image] $=$ erosion[dilation[binary image]]

The window shape is important because it affects the changes induced in binary objects; in addition to being simply square or rectangular, it can be a plus shape, a 
Table 4.2: Binary Image B

\begin{tabular}{|l|l|l|l|l|l|l|l|}
\hline 0 & 0 & 0 & 0 & 0 & 0 & 0 & 0 \\
\hline 1 & 1 & 1 & 1 & 1 & 1 & 1 & 0 \\
\hline 0 & 0 & 0 & 1 & 1 & 1 & 1 & 0 \\
\hline 0 & 0 & 0 & 1 & 1 & 1 & 1 & 0 \\
\hline 0 & 0 & 1 & 1 & 1 & 1 & 1 & 0 \\
\hline 0 & 0 & 0 & 1 & 1 & 1 & 1 & 0 \\
\hline 0 & 0 & 1 & 1 & 0 & 0 & 0 & 0 \\
\hline 0 & 0 & 0 & 0 & 0 & 0 & 0 & 0 \\
\hline
\end{tabular}

Table 4.3: Closing $B \bullet S$

\begin{tabular}{|l|l|l|l|l|l|l|l|}
\hline 0 & 0 & 0 & 0 & 0 & 0 & 0 & 0 \\
\hline 0 & 1 & 1 & 1 & 1 & 1 & 1 & 0 \\
\hline 0 & 0 & 1 & 1 & 1 & 1 & 1 & 0 \\
\hline 0 & 0 & 1 & 1 & 1 & 1 & 1 & 0 \\
\hline 0 & 0 & 1 & 1 & 1 & 1 & 1 & 0 \\
\hline 0 & 0 & 1 & 1 & 1 & 1 & 1 & 0 \\
\hline 0 & 0 & 1 & 1 & 0 & 0 & 0 & 0 \\
\hline 0 & 0 & 0 & 0 & 0 & 0 & 0 & 0 \\
\hline
\end{tabular}


diagonal shape, or in fact, any desired pattern. In morphological image processing, the shaped window is called a structuring element and can be designed to perform pattern matching or modification of particular shapes [15].

\subsection{Quantitative Analysis}

To recognize and locate the direction of binary objects, we study the geometric properties like area and orientation.

Let

$$
\begin{gathered}
I_{i}(r, c)=1 ; \text { if } I(r, c)=i^{\text {th }} \text { objectnumber } \\
=0 ; \text { otherwise }
\end{gathered}
$$

The area of the $i^{t h}$ object is given as $A_{i}=\Sigma_{r=0}^{N-1} \Sigma_{c=0}^{N-1} I_{i}(r, c)$.

This indicates the relative size of the object and is measured in pixels. Based on the spatial distribution of the pixels, the centroid or the center of area $\left(\bar{r}_{i}, \bar{c}_{i}\right)$ are defined as

$$
\begin{gathered}
\bar{r}_{i}=\frac{1}{A_{i}} \sum_{r=0}^{N-1} \sum_{c=0}^{N-1} r I_{i}(r, c), \\
\bar{c}_{i}=\frac{1}{A_{i}} \sum_{r=0}^{N-1} \sum_{c=0}^{N-1} c I_{i}(r, c)
\end{gathered}
$$

This feature helps to locate an object in the two-dimensional image plane.

The axis of least second moment or axis of least inertia corresponds to the line about which it takes the least amount of energy to spin an object of like shape. This provides information about the object's orientation. If the origin is moved to the center of area $\left(r_{i}, c_{i}\right)$, the axis of least second moment is given by

$$
\tan 2 \theta_{i}=\frac{2 \Sigma_{r=0}^{N-1} \Sigma_{c=0}^{N-1} r c I_{i}(r, c)}{\Sigma_{r=0}^{N-1} \Sigma_{c=0}^{N-1} r^{2} I_{i}(r, c)-\Sigma_{r=0}^{N-1} \Sigma_{c=0}^{N-1} c^{2} I_{i}(r, c)}
$$


Figure 4.4 shows the angle of least second moment [26]. The angle $\theta$ is measured in counter clock-wise direction from r-axis.

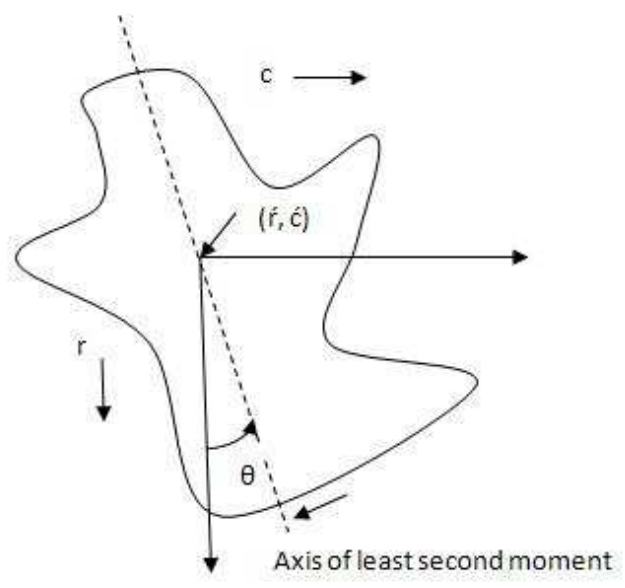

Figure 4.4: Computation of angle of least inertia 


\section{Chapter 5}

\section{Experiments and Results}

This chapter provides the experimental results obtained from the spatio-spectral processing techniques for dust storm detection and finding direction. The qualitative and quantitative results obtained by these processing schemes on three different raw data sets is provided. The data sets are from April 15, 2003, December 15, 2003 and April 1, 2003. The results achieved by processing these images in environment for visualizing images (ENVI) software package and MATLAB are shown.

The first data set is from April 15, 2003. Figure 5.1 shows the cropped $512 \times 512$ dust storm region of band 4 .

Power spectrum analysis is done by block processing on this image to confirm the presence of high directionality information in certain regions of an image where the dust storm is present. Figure 5.2 shows the power spectrum of $16128 \times 128$ blocks. The blocks which are marked in red indicate the presence of dust storm.

Binary thresholding is done on the power spectrum based on Otsu's method to enhance the directional texture and to be able to study the direction. Figure 5.3 shows the binary thresholding on the power spectrum.

Image closing operation is then performed on the binary thresholded image in 


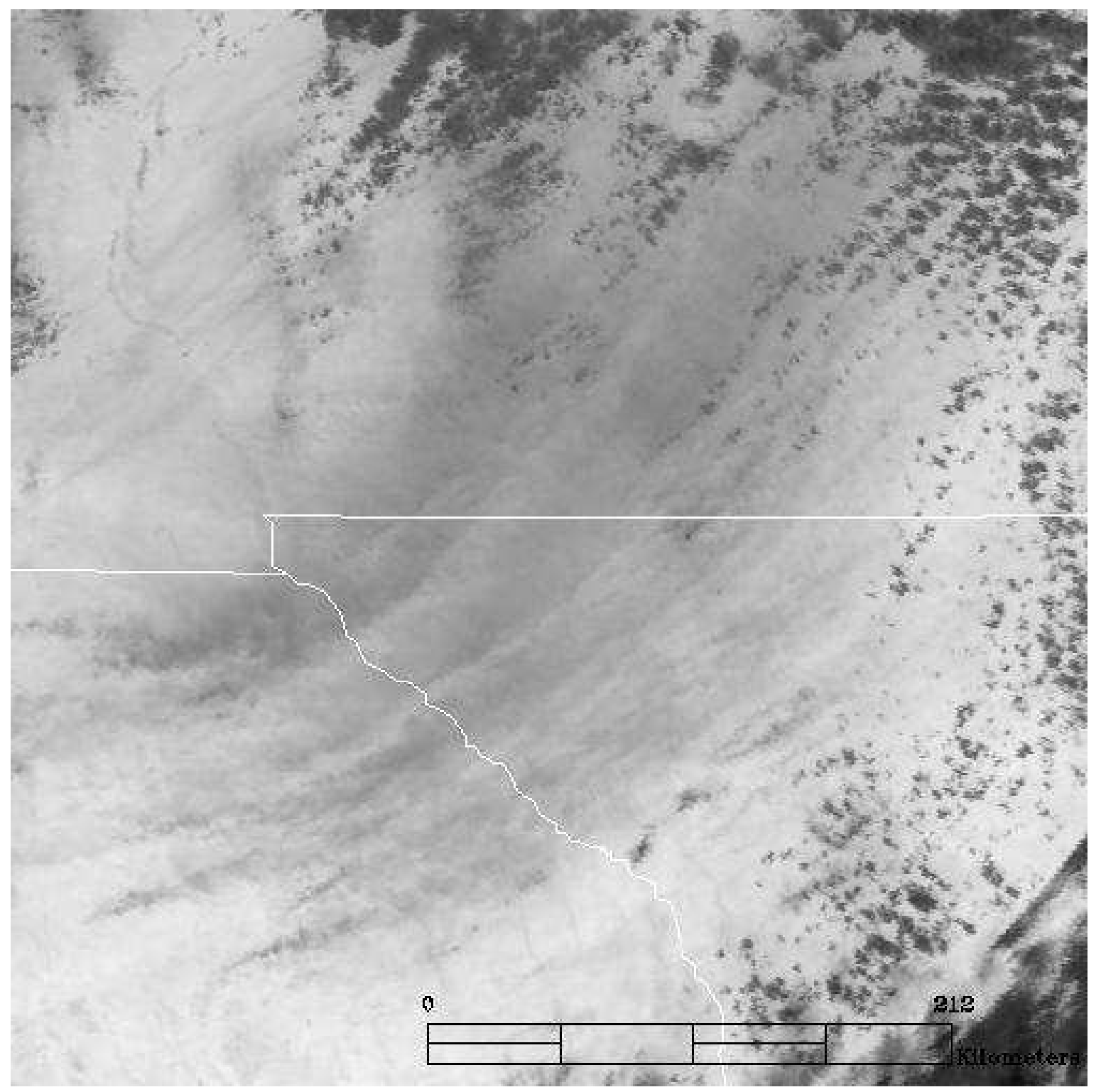

Figure 5.1: Cropped 512x512 band 4 of April 15, 2003 event 


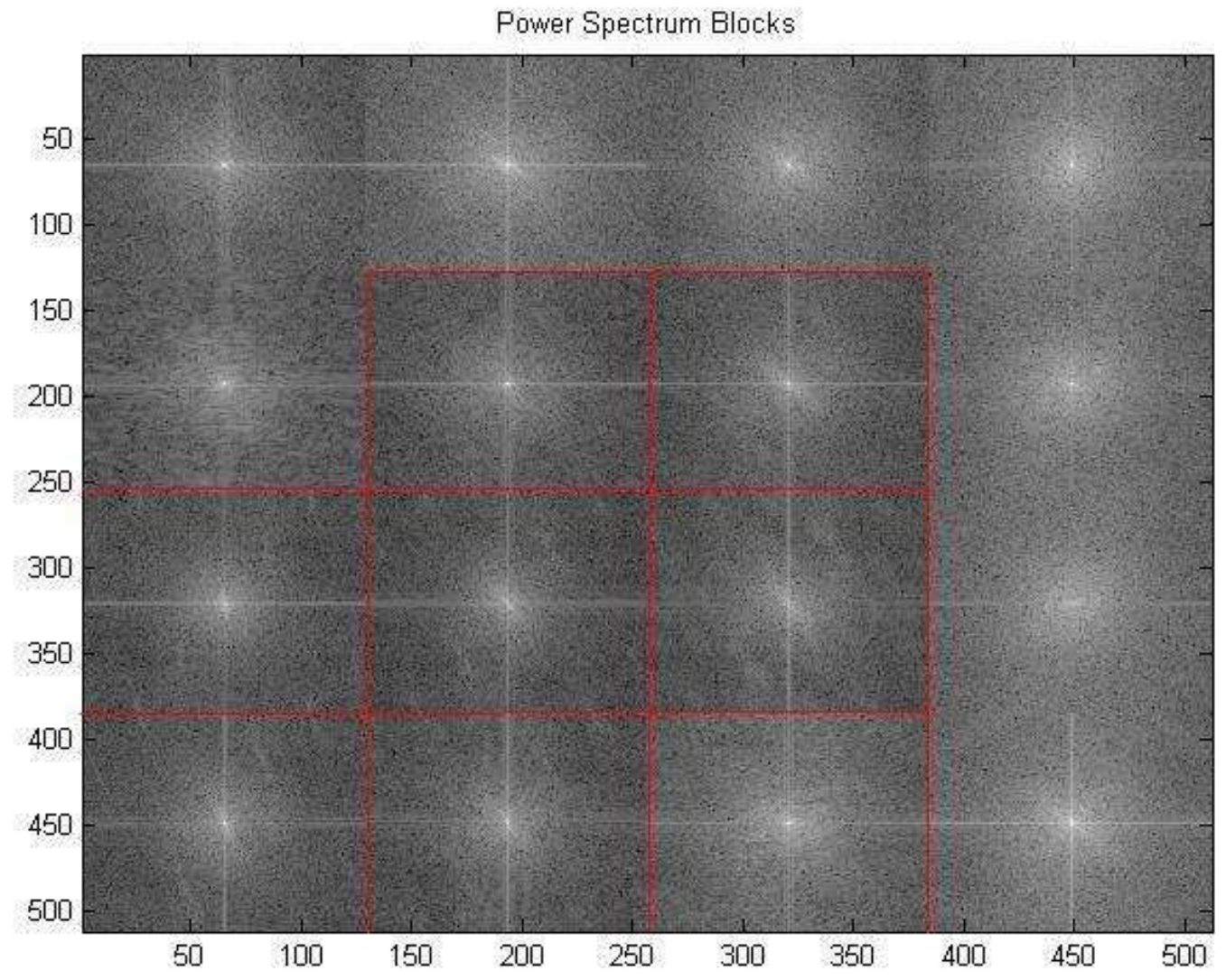

Figure 5.2: Power Spectrum Analysis of April 15, 2003 event (square of magnitude of DFT) 


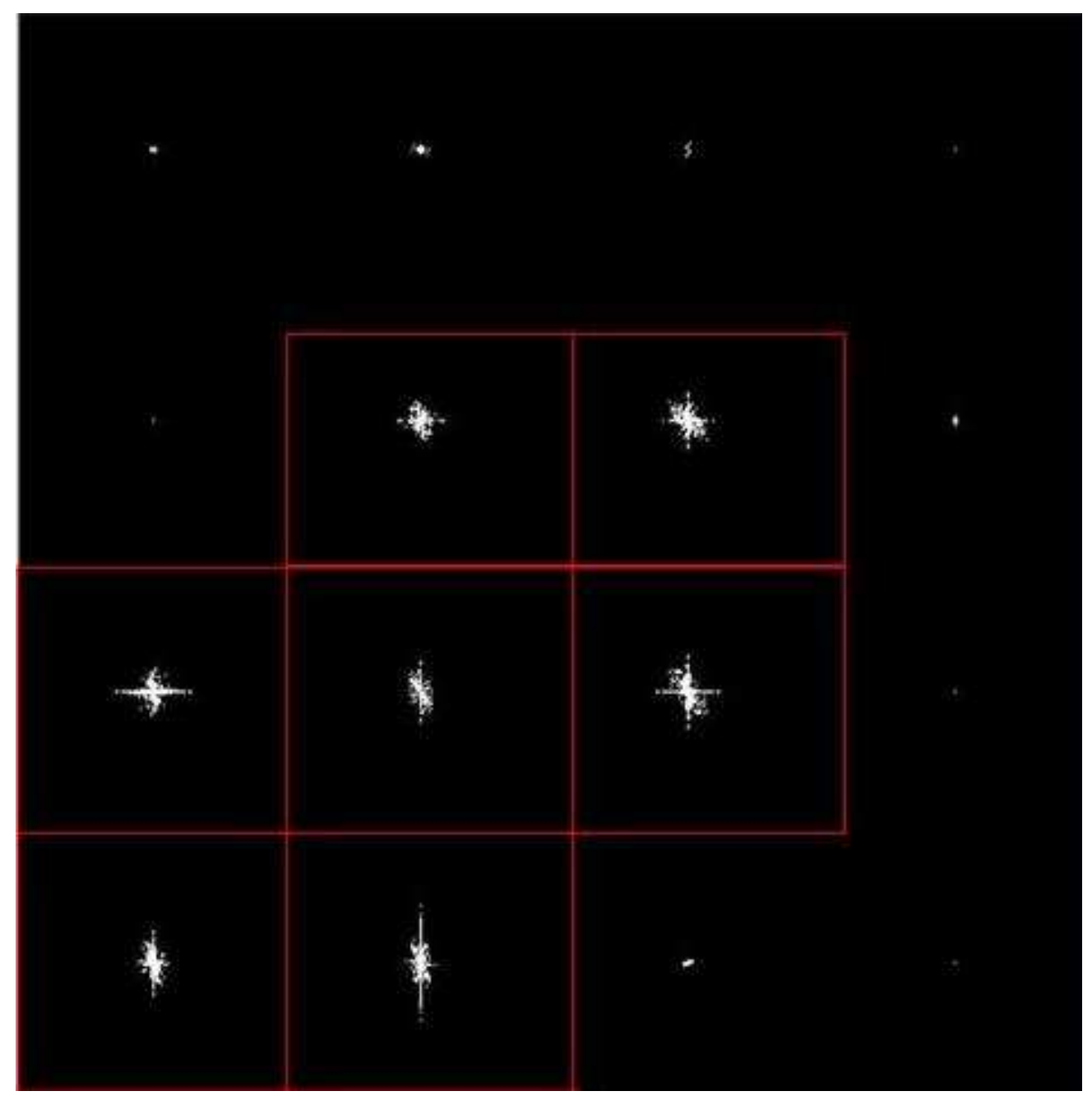

Figure 5.3: Binary thresholding of the powerspectrum of April 15, 2003 event 
order to extract the shape and geometry of the binary objects in the image. Figure 5.4 shows the morphologically enhanced blocks of the binary image.

The quantitative analysis is done by finding the area and orientation of these binary objects. It was observed that, if the area of the binary objects is more than one percent of the total size of the image indicating the presence of dust storm. Figure 5.5 shows the area and orientation computations of the binary objects in the morphologically enhanced image. The orientation values are orthogonal to the direction of dust storm in spatial domain. Figure 5.6, 5.7, 5.8 show the power spectrum analysis (square root of magnitude of DFT), binary thresholding and morphological enhancements.

The following are the results obtained from the automation technique for December 15, 2003 event. Figure 5.9 shows the cropped 512x512 dust storm region of April 15th, 2003 dust event. Figure 5.10 shows the power spectrum of sixteen of 128x128 blocks. Figure 5.11 shows the binary thresholding on the power spectrum. Figure 5.12 shows the morphologically enhanced blocks of the binary image. Figure 5.13 shows the area and orientation computations of the binary objects in the morphologically enhanced image. The orientation values are orthogonal to the direction of dust storm in spatial domain.

To analyze false alarms, we study two data sets which have no dust storm in them. The first one is the April 1, 2003 event. The second one is the cropped non-dust storm region from the December 15, 2003.

The April 1, 2003 event is analyzed to view the performance of the proposed technique on non-dust storm events. These results are used to analyze false alarms that occur during the absence of dust storm. The visualization technique does not segment dust storm region by band math analysis. Figure 5.14 shows the bandmath results (band 4 - band 5) for April 1st, 2003 dust event. The marked dark region is not a dust storm, but is picked up by band math analysis. So, this suspected $512 \times 512$ 


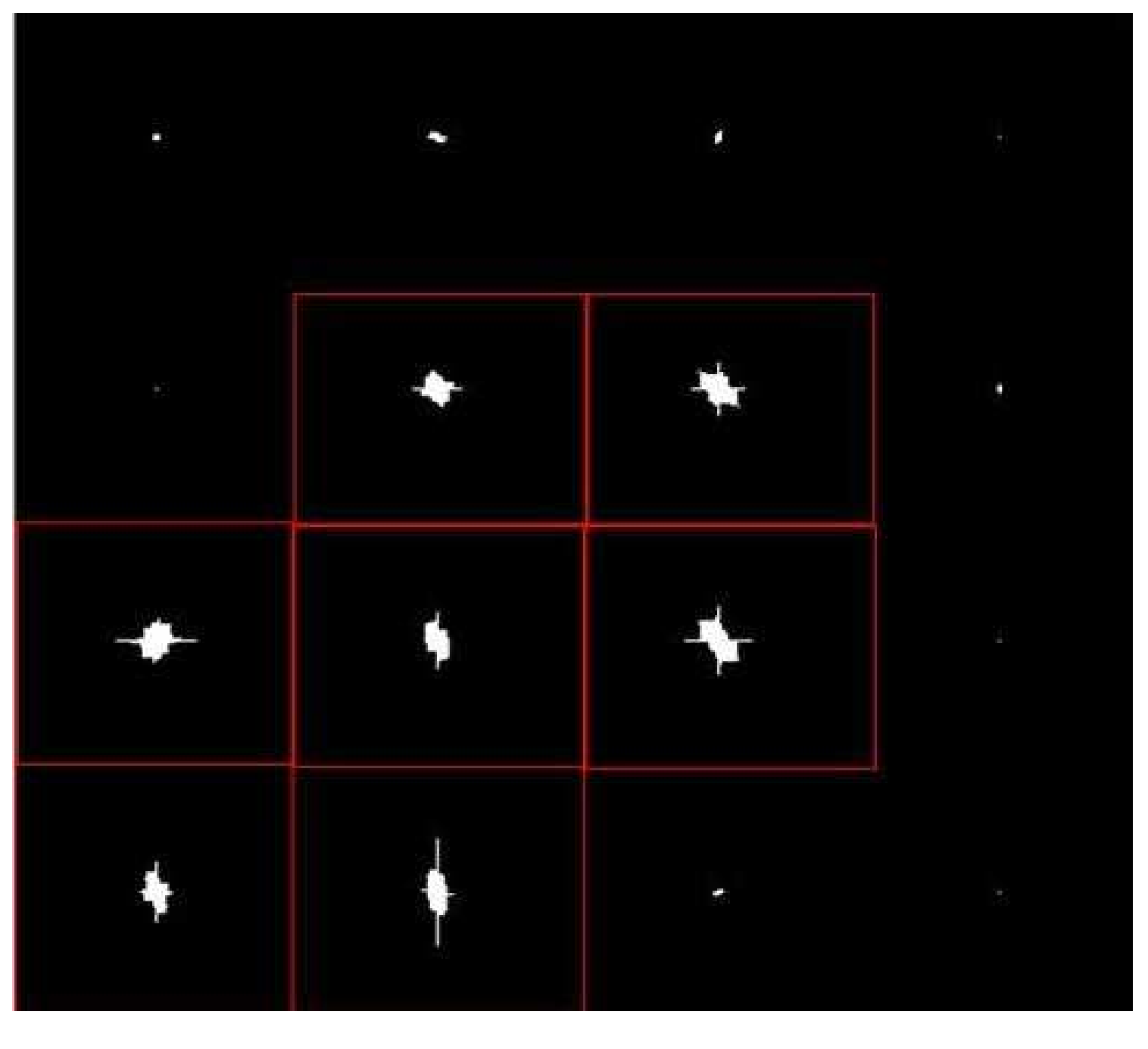

Figure 5.4: Morphological enhancement on the binary thresholded image of April 15, 2003 event 


\begin{tabular}{|c|c|c|c|}
\hline 9 & 32 & 15 & 1 \\
\hline $0^{\circ}$ & $-20.9101^{\circ}$ & $73.1550^{\circ}$ & $0^{\circ}$ \\
\hline 1 & 224 & 413 & 9 \\
\hline $0^{\circ}$ & $-25.7262^{\circ}$ & $-52.4462^{\circ}$ & $90^{\circ}$ \\
\hline 437 & 221 & 580 & 1 \\
\hline $21.8736^{\circ}$ & $-78.4846^{\circ}$ & $-57.6550^{\circ}$ & $0^{\circ}$ \\
\hline 267 & 438 & 12 & 1 \\
\hline$-79.3580^{\circ}$ & $-87.5584^{\circ}$ & $20.3006^{\circ}$ & $0^{\circ}$ \\
\hline
\end{tabular}

Figure 5.5: Area and Orientation Computations of April 15, 2003 event 
Power Spectrum Blocks

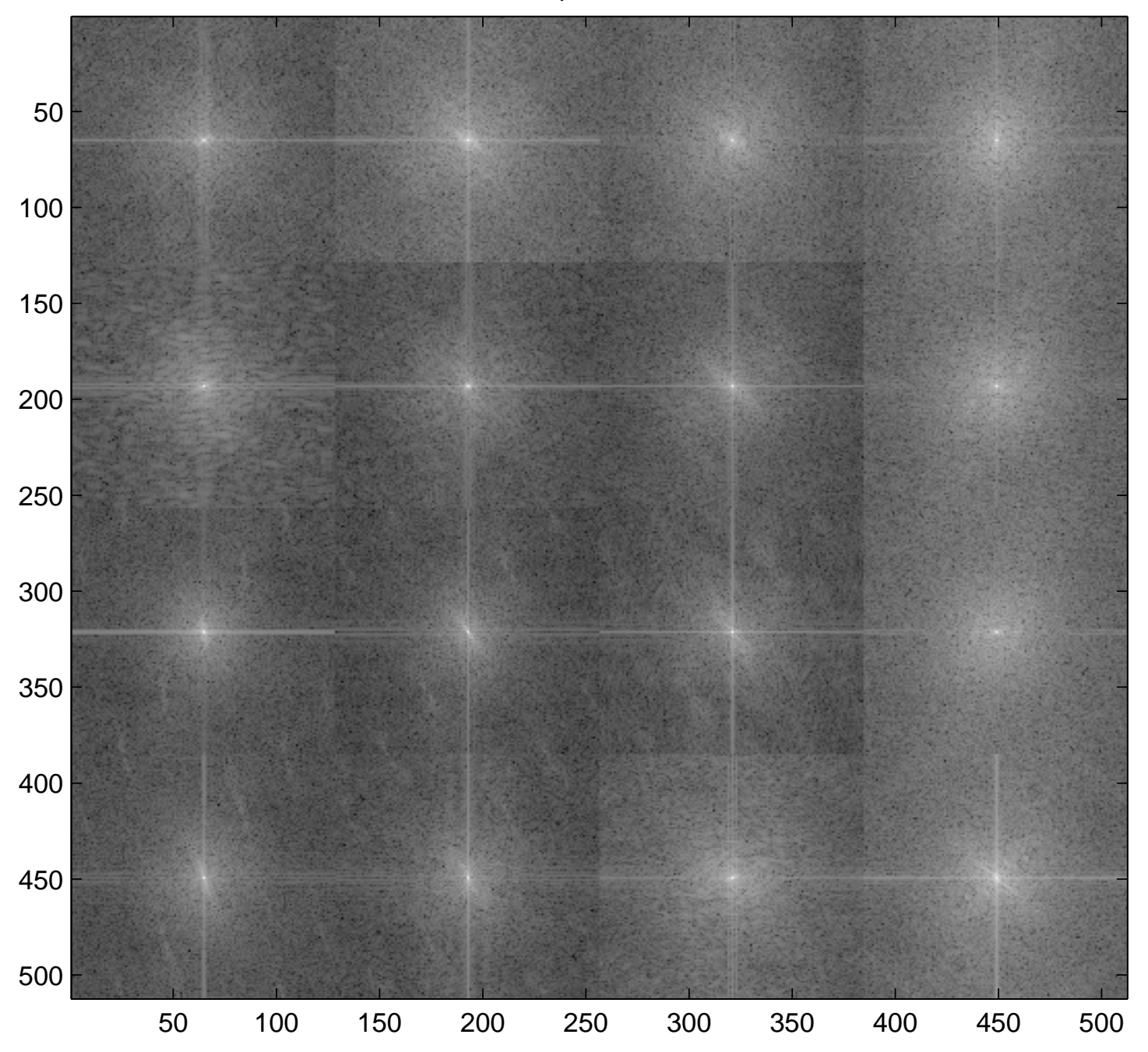

Figure 5.6: Power Spectrum Analysis of April 15, 2003 event (square root of magnitude of DFT) 
Binary Thresholded

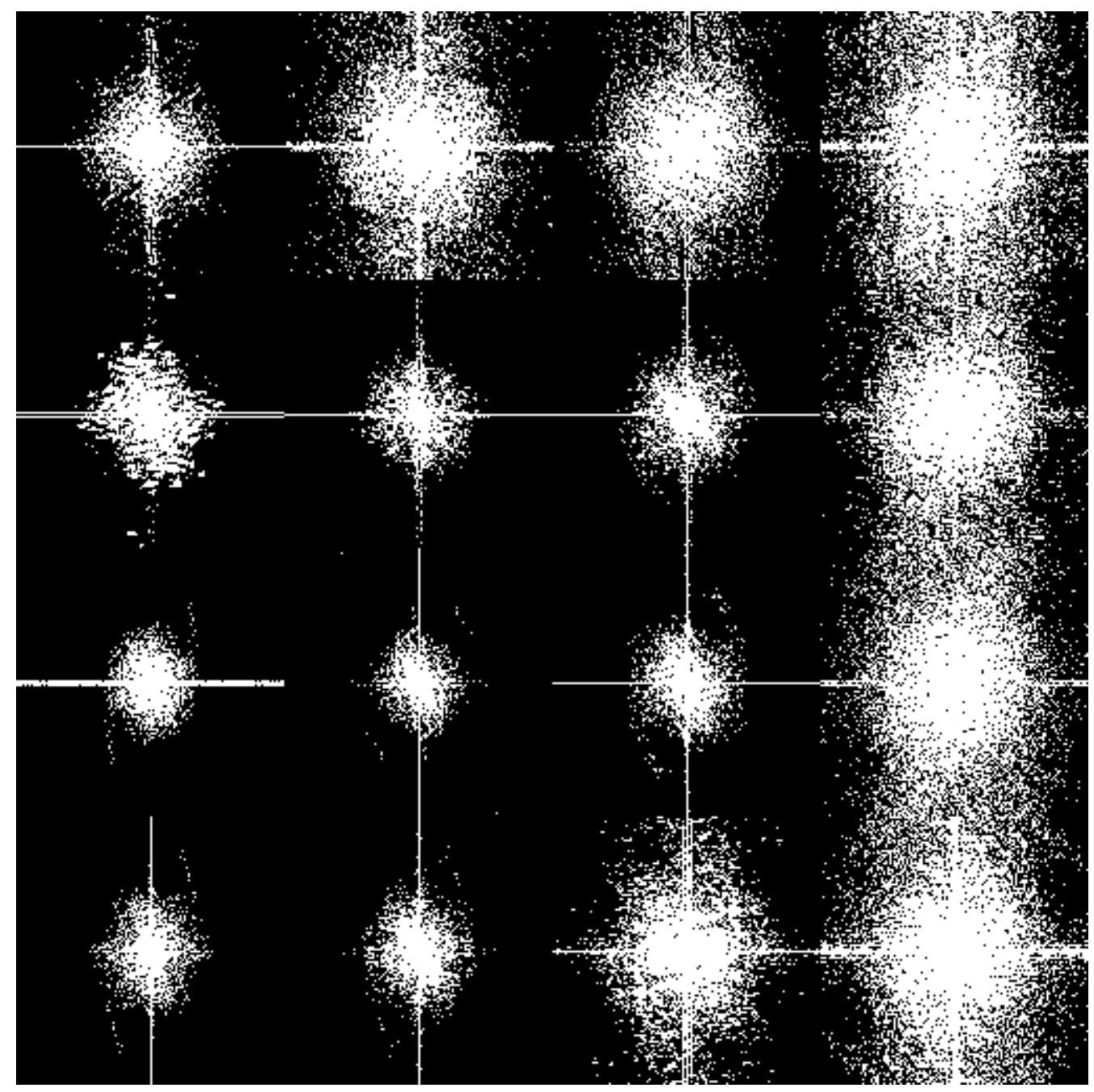

Figure 5.7: Binary thresholding of the powerspectrum of April 15, 2003 event 


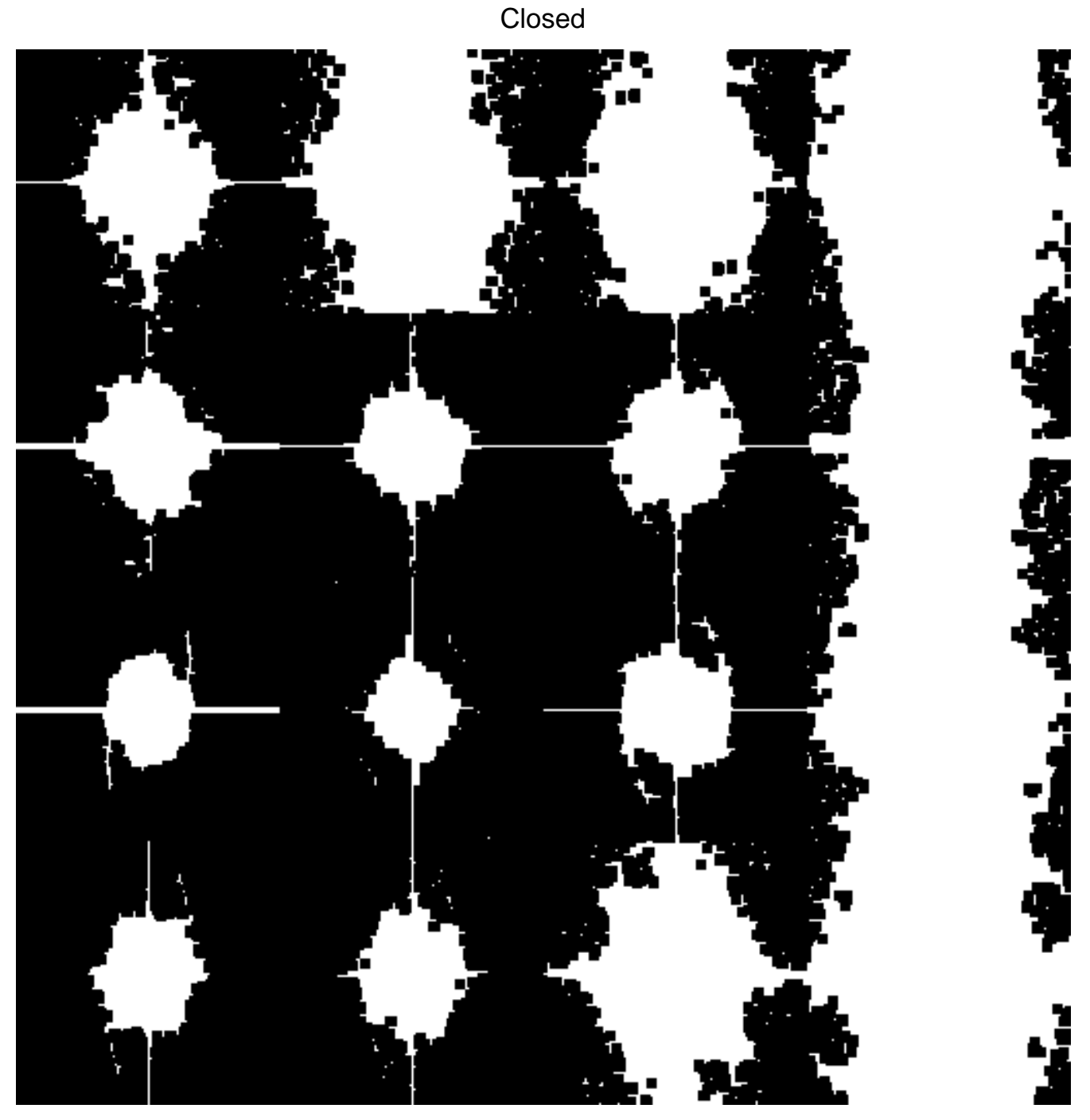

Figure 5.8: Morphological enhancement on the binary thresholded image of April 15, 2003 event 


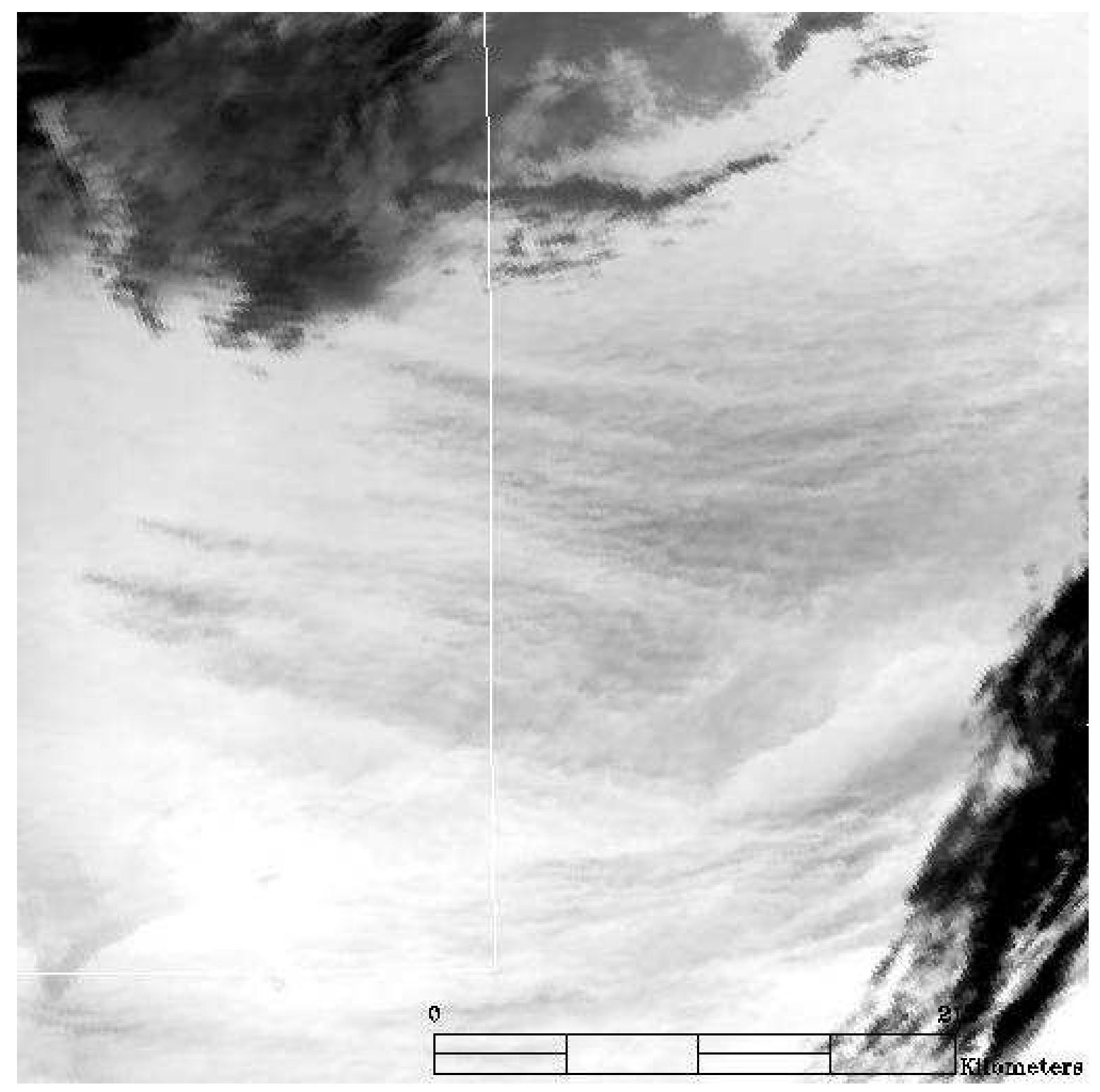

Figure 5.9: Cropped 512x512 band 4 of December 15, 2003 event 


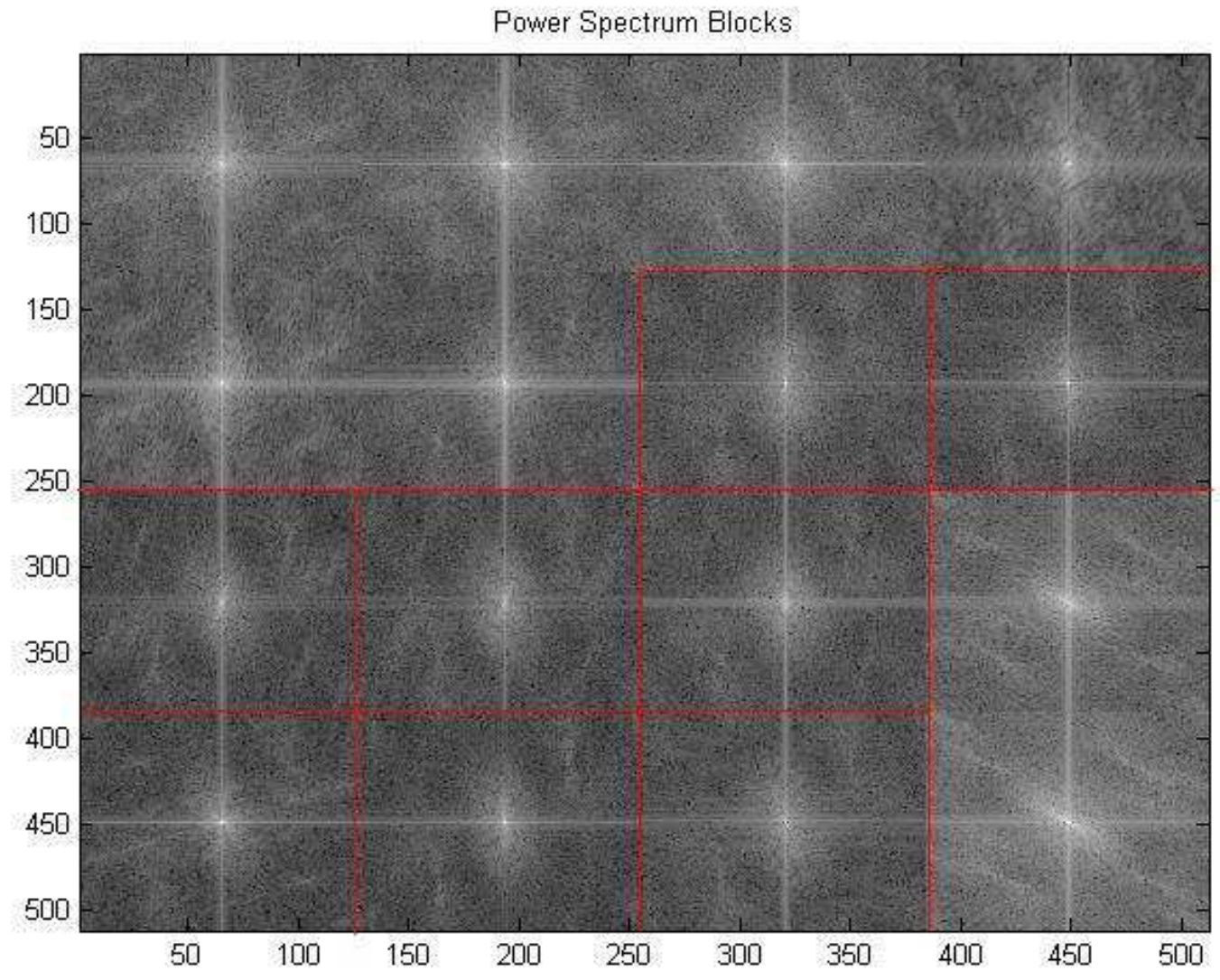

Figure 5.10: Power Spectrum Analysis of December 15, 2003 event 


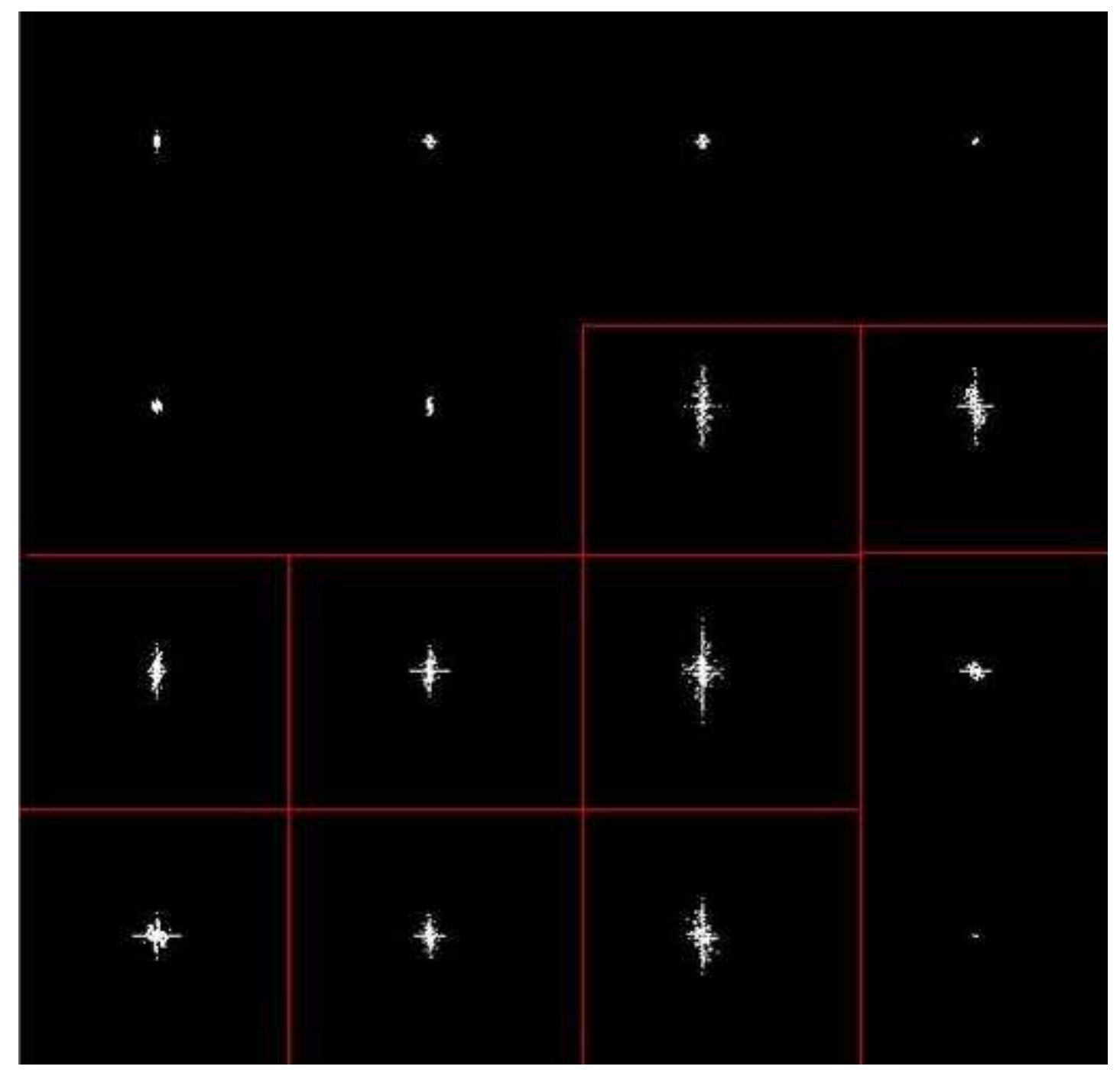

Figure 5.11: Binary thresholding of the powerspectrum of December 15, 2003 event 


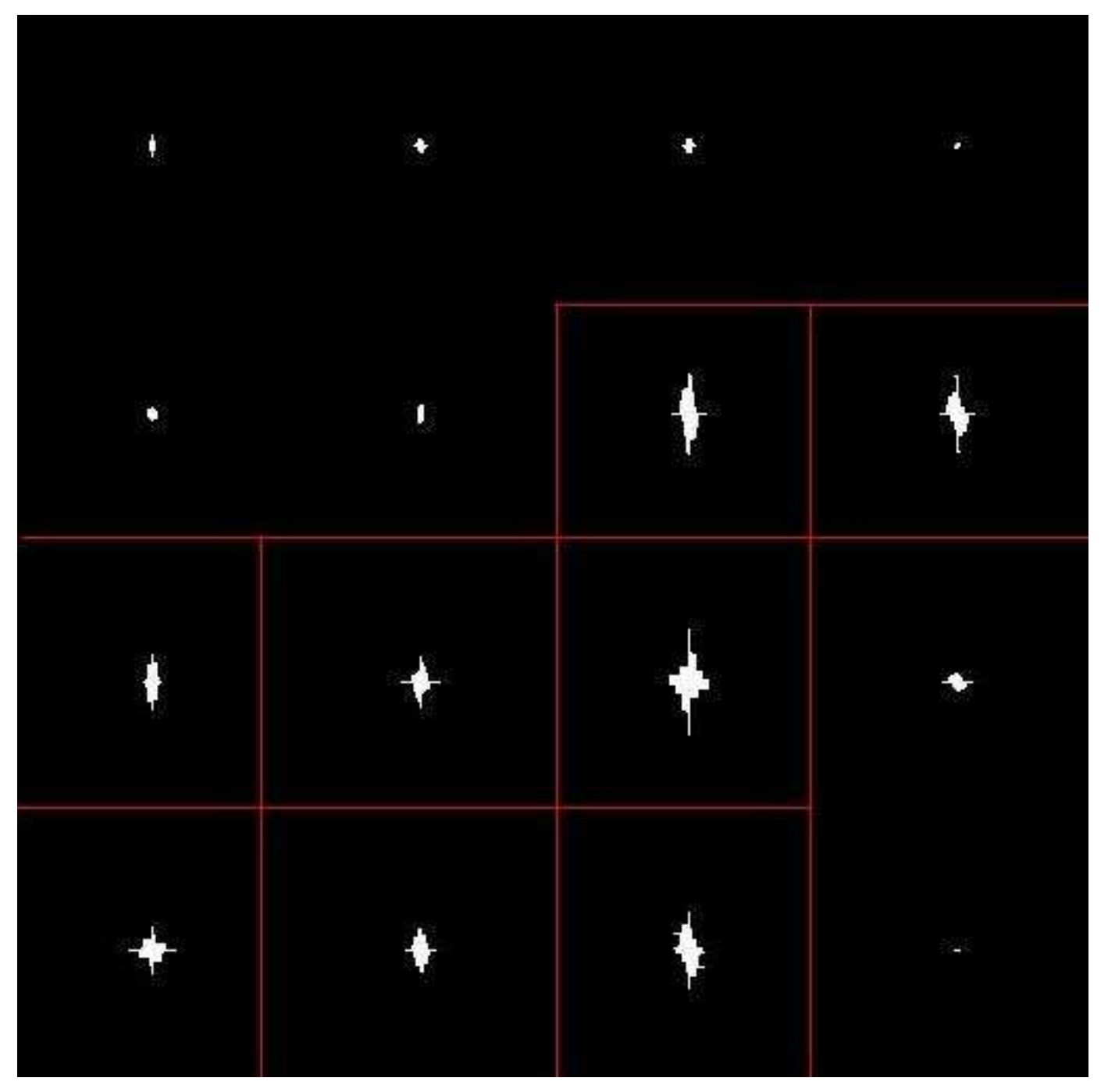

Figure 5.12: Morphological enhancement on the binary thresholded image of December 15, 2003 event 


\begin{tabular}{|c|c|c|c|}
\hline 25 & 29 & 33 & 7 \\
\hline $90^{\circ}$ & $-68.8632^{\circ}$ & $84.0557^{\circ}$ & $45^{\circ}$ \\
\hline 26 & 25 & 354 & 344 \\
\hline$-69.1832^{\circ}$ & $86.5444^{\circ}$ & $-88.0392^{\circ}$ & $-82.5465^{\circ}$ \\
\hline 147 & 241 & 537 & 77 \\
\hline $88.8623^{\circ}$ & $85.9770^{\circ}$ & $87.3790^{\circ}$ & $-17.5297^{\circ}$ \\
\hline 265 & 172 & 338 & 3 \\
\hline$-16.8450^{\circ}$ & $-85.7150^{\circ}$ & $20.3006^{\circ}$ & $0^{\circ}$ \\
\hline
\end{tabular}

Figure 5.13: Area and Orientation Computations of December 15, 2003 event 
region is cropped for false alarm analysis.

The cropped 512x512 dust storm region of April 1st, 2003 dust event and the results of k-means classification are shown. As shown in Figure 5.16, k-means classification is not able to well segment the same false-alarm region. So, we need not go into the next step of automation technique for finding the dust storm direction as illustrated in the flowchart in Figure 2.2.

Another example of analyzing false alarm is studied using non-dust storm region of December 15, 2003 data set. Bandmath analysis i.e, band 4 - band 5 is performed on the raw data set. A $512 \times 512$ region which does not contain dust storm is cropped from the resultant image. Now, we apply our spatio-spectral processing scheme to this non-dust storm image. The first step is to perform bandmath analysis on this $512 \times 512$ image to see if the bandmath is able to pick up any dust storm. Figure 5.17 shows the result of bandmath analysis.

The resultant image does not pick up any darker regions as expected. Therefore, we do not proceed with further steps of locating dust sources or finding the direction of dust storm. 


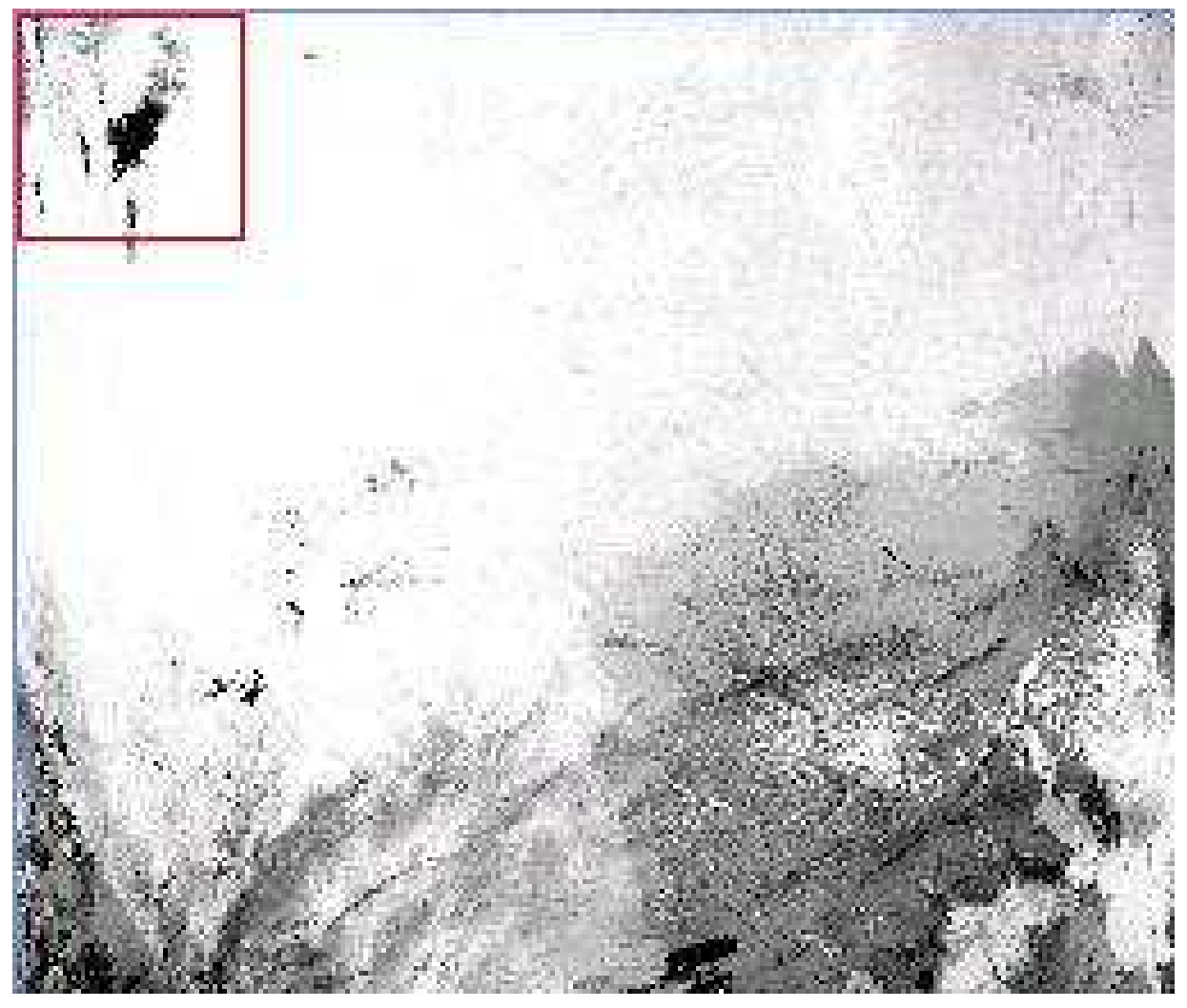

Figure 5.14: Bandmath image of April 1, 2003 event 


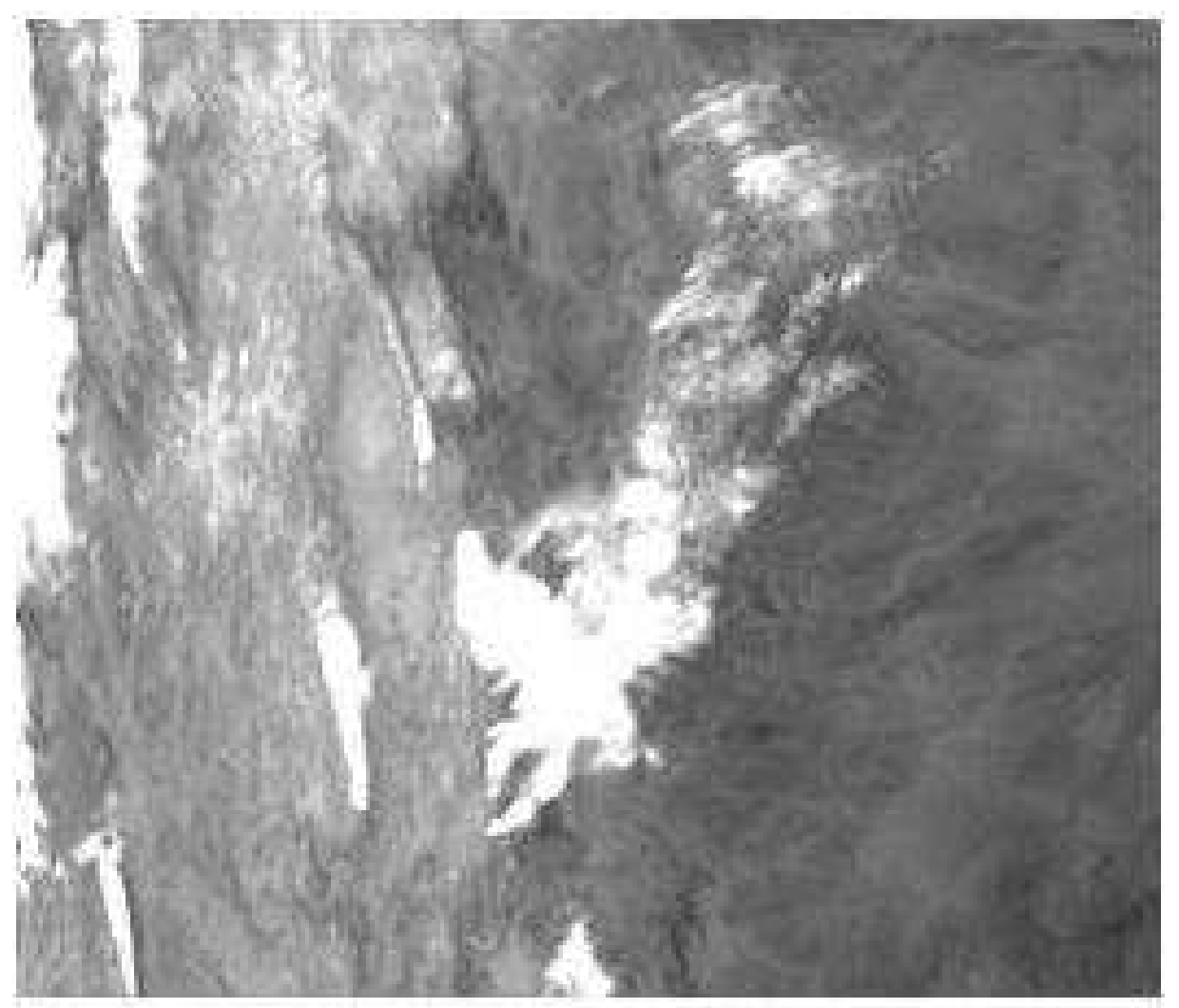

Figure 5.15: Original 512x512 image of April 1, 2003 


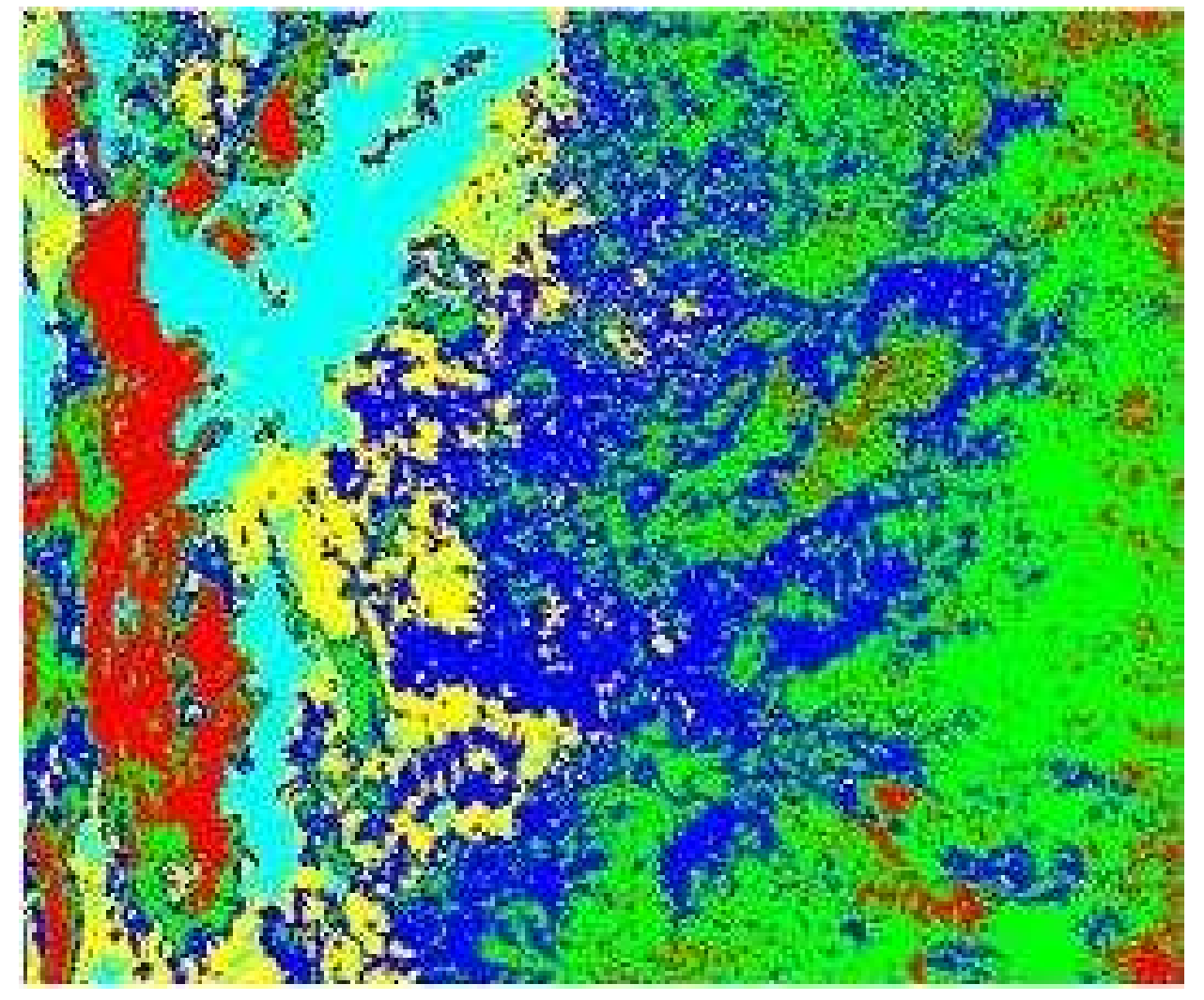

Figure 5.16: k-means classified 512x512 image of April 1, 2003 


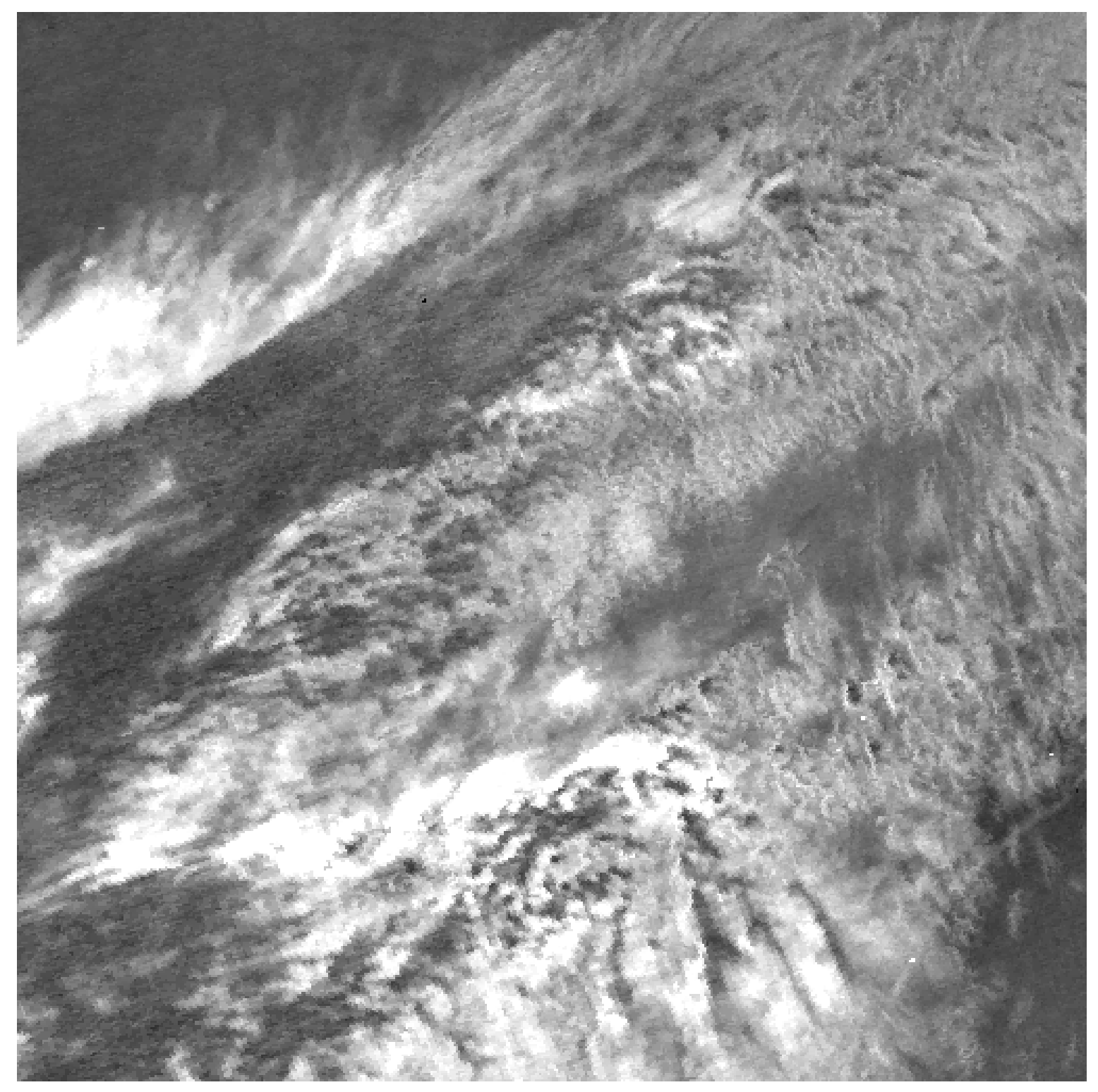

Figure 5.17: Bandmath result of cropped 512x512 non-dust storm image of December 15, 2003 


\section{Chapter 6}

\section{Conclusion and Future Work}

The proposed spatio-spectral processing scheme in this research detects the presence of dust storm and automates the process of finding dust storm direction in NOAAAVHRR images. The visualization technique for dust storm detection confirms its presence of dust storm and locates the origin of dust sources using directional filtering and edge detection techniques. Results from k-means classification and spectral transforms like PCA and MNF are compared with the results from band math analysis to confirm the presence of a dust storm.

The automation technique is intended to detect the presence of dust storm and find the dust storm direction. Band 4 and Band 5 are used in this technique because of their significance in detecting silicate particles. A 512x512 image is cropped in band 4 and band 5 based on results from visualization technique. Block processing is performed on $128 \times 128$ blocks of this image. Power spectrum analysis is done on these 16 blocks followed by binary thresholding and morphological enhancement. Power spectrum indicates the presence of dust storm showing evidence of directional texture in some blocks where the dust storm is present. This prominent direction in the frequency domain is orthogonal to the highest directional components in the image 
in the spatial domain. These blocks are considered as candidate regions for dust storm detection. Binary thresholding is applied to reveal stronger directional energy and indicate binary objects in these blocks. Morphological enhancement makes it suitable for shape and geometric property analysis of binary objects in an image. Area and orientation are computed on all the blocks of morphologically enhanced images. If the area of the $128 \times 128$ block is more than one percent of the total area of the $512 \times 512$ image, it indicates the presence of dust storm. Orientation of binary objects in these blocks is calculated to detect the dust storm direction. Since, this orientation angle is computed based on the power spectrum image, we calculate the orthogonal direction to these resultant angles to find the actual direction of dust storm in these blocks.

Similar bandmath analysis (band 4 - band 5 for NOAA-AVHRR imagery) was also used to detect volcanic ash eruptions in [30]. Therefore, the proposed spatiospectral technique may be used to perform volcanic ash tracking. Some automated techniques have been proposed for detecting tornadoes and volcanoes operationally [29], [31]. The proposed processing technique could aid in providing timely warnings of dust storm regarding implications or hazard assessments for transportation, aviation, environmental safety and public health.

The spatio-spectral processing technique proposed in this research is one possible approach for directional analysis of dust storm. Future work may targeted towards more quantitative and qualitative results demonstrating directionality information of the transport of dust storms. Further analysis on the enhanced directional streaks resulting from visualization technique could be done to see if they further correspond to the dust plumes, when followed for longer distances. Performance of the technique for monitoring volcanic ash could also be considered. The behavior and properties of volcanic ash particles determine the significant bands that can be used for further analysis. Possible investigation of more advanced multi-resolution directional filtering like Wavelets, and Bamberger pyramids is recommended to detect the dust storm 
direction and locate the dust sources. Figure 6.1 shows preliminary experimental results obtained from the Discrete Wavelet Transform(DWT). The energy of subbands in the region of dust storm seems to be much lower than the energy of subbands in the non-dust storm regions.

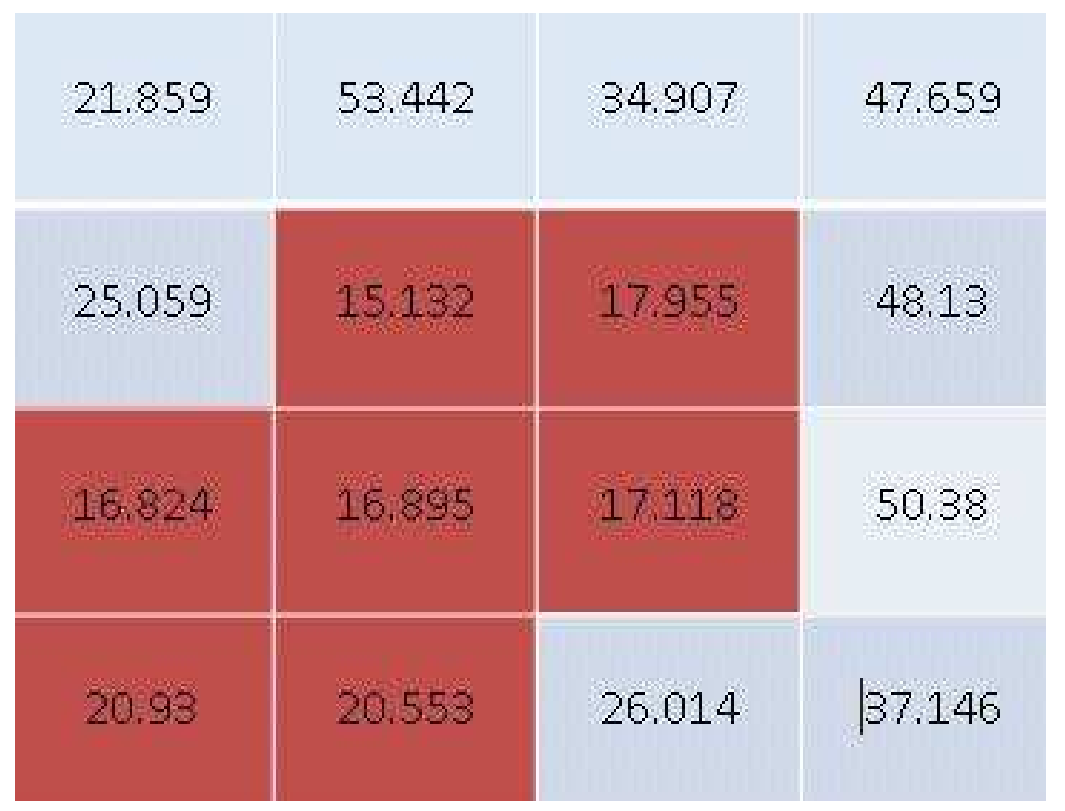

Figure 6.1: Energy of subbands for cropped 512x512 band 4 of April 15, 2003 event

The proposed future work also includes the assessment of this processing technique on the GOES and MODIS images, which are more frequently available so that it could be used operationally to automatically detect dust storms. These processing techniques may also be evaluated on the images with no dust storm and during different times of day to analyze false alarms. False alarms that might result from the textures of clouds, smoke, fire and air pollution may also be studied. Further investigation on supervised and unsupervised classification techniques is also suggested. 


\section{Bibliography}

[1] Nancy I. Rivera Rivera, "Detection and characterization of dust source areas in the Chihuahuan desert, Southwestern North America" Masters Thesis, University of Texas at El Paso, December 2006.

[2] Jeffrey A.Lee, Thomas E. Gill, Kevin R.Mulligan, Miguel Domniguez Acosta, Adriana E.Perez, "Land use/land cover and point sources of the 15 December 2003 dust storm in southwestern North America", Geomorphology, doi: 10.1016/j.geomorph.2007.12.016.

[3] Hesham El-Askary, Menas Kafatos, Xue Liu, Tarek El-Ghazawi, "Introducing new approaches for dust storms detection using remote sensing technology",Proceedings of IEEE International Geoscience and Remote Sensing Symposium IGARSS03, France, July 2003.

[4] Nahid Khazenie, Thomas F. Lee, "Identification of aerosol features such as smoke and dust, in NOAA-AVHRR data using spatial textures", Proceedings of IEEE International Geoscience and Remote Sensing Symposium IGARSS92, Houston, Texas, May 1992.

[5] Steven A. Ackerman, "Remote sensing aerosols usign satellite infrared observations", Journal of Geophysical Research, vol. 102, no. D14 pp. 17069-17079, 1997. 
[6] Abhishek Agarwal, Hesham El-Askary, Tarek El-Ghazawi, Menas Kafatos, and Jacqueline Le-Moigne "Hierarchial PCA techniques for fusing spatial and spectral observations with application to MISR and monitoring dust storms", IEEE Geoscience and Remote Letters, vol. 4, issue. 4 pp. 678-682, October 2007.

[7] Nahid Khazenie, Thomas F. Lee, "Automatic detection of dust storm in the north west of China using decision tree classifier based on MODIS visible bands data", Proceedings of IEEE International Geoscience and Remote Sensing Symposium IGARSS05, Korea, July 2005.

[8] David A. Landgrebe, Signal Theory Methods in Multispectral Remote Sensing, Wiley-Interscience Publishers, 2003, ISBN 0-471-42028-X.

[9] P. S. Chavez, D. J. Mackinnon, R. L. Reynolds and M. G. Velasco, "Monitoring dust storms and mapping landscape vulnerability to wind erosion using satellite and ground-based digital images", Aridlands News Letter, no. 51, May / June 2002.

[10] C. K. Wentworth, "A scale of grade and class terms for clastic sediments", Journal of Geology, vol. 30, pp. 377-392, 1922.

[11] Andrew A. Green, Mark Berman, Paul Switzer and Maurice D. Craig, "A transformation for ordering multispectral data in terms of image quality with implications for noise removal", IEEE Transcations on Geoscience and Remote Sensing, vol. 26, issue. 1, pp.65-74, January 1988.

[12] J. W. Boardman, and F. A. Kruse, "Automated spectral analysis: a geological example using AVIRIS data, north Grapevine Mountains, Nevada", Proceedings of ERIM Tenth Thematic Conference on Geologic Remote Sensing, Environmental Research Institute of Michigan, Ann Arbor, MI, pp.I-407 - I-418, 1994. 
[13] N. Otsu, "A Threshold Selection Method from Gray-Level Histograms", IEEE Transactions on Systems, Man, and Cybernetics, vol. 9, no. 1, pp.62-66, 1979.

[14] William T. Freeman, Edward H. Adelson, "The Design and Use of Steerable Filters", IEEE Transactions on Pattern Analysis and Machine Intelligence, vol. 13, no. 9, September 1991.

[15] Robert A. Schowengerdt, Remote Sensing : Models and methods for Image Processing, Third Edition, Academic Press, 2007, ISBN-13:978-0-12-369407-2, ISBN-10:0-12-369407-8.

[16] Milan Sonka, Vaclav Hlavac, Roger Boyle, Image Processing, Analysis, and Machine Vision, Thomson, 2008, ISBN-13:978-0-495-08252-1, ISBN-10:0-495-08252$\mathrm{X}$.

[17] Scott E Umbaugh, "Computer Imaging : Digital Image Analysis and Processing", CRC Press, 2005, ISBN 0-8493-2919-1.

[18] Vikram Jayaram, "Detection from Hyperspectral images compressed using rate distortion and optimization techniques under JPEG2000 Part2", Masters Thesis, University of Texas at El Paso, November 2004.

[19] F.A. Kruse, J.W. Boardman, A.B. Lefkoff, K.B. Heidebrecht, A.T. Shapiro, P.J. Barloon, and A.F.H. Goetz, "The Spectral Image Processing System (SIPS): Interactive visualization and analysis of imaging spectrometer data", AIP Conference Proceedings, vol. 283, pp. 192-201, 1993.

[20] Richard O. Duda, Peter E. Hart, David G. Stork, "Pattern Classification", WileyInterscience, 2001, ISBN 0-471-05669-3.

[21] Christopher M. Bishop, "Pattern Recognition and Machine Learning", Springer, 2006, ISBN 0-387-31073-8. 
[22] Robert A. Schowengerdt, "Techniques for Image Processing and Classification in Remote Sensing", Academic Press, 1983, ISBN 0-12-628980-8.

[23] R. Michael Hord, "Digital Image Processing of Remotely Sensed Data", Academic Press, 1982, ISBN 0-12-355620-1.

[24] R. Jayant and P. Noll, "Digital Coding of Waveforms", Upper Saddle River, NJ: Prentice Hall, 1984.

[25] Anil. K. Jain, "Fundamentals of Digital Image Processing", Upper Saddle River, NJ: Prentice Hall, 1989, ISBN 0-13-336165-9.

[26] Linda G. Shapiro, George. C. Stockman "Computer Vision", Upper Saddle River: Prentice Hall, 2001, ISBN 0-13-030796-3.

[27] Rafael C. Gonzalez, Richards E. Woods, "Digital Image Processing", Pearson Education, 2002, ISBN 81-7808-629-8.

[28] John R. Jensen, "Remote Sensing of the Environment : An Earth Resource Perspective", Pearson Education, 2007, ISBN 0-13-188950-8.

[29] P.W. Webley, D. Atkinson, R.L. Collins, K.Dean, J. Fochesatto, K.Sassen, C.F. Cahill, A. Prata, C.J. Flynn, K.Mizutani, "Predicting and validating the tracking of a volcanic ash cloud during the 2006 eruption of Mt.Augustine Volcano", BAMS, vol. 89, issue. 11, pp. 1647-1658, November 2008.

[30] F. Prata"Observations of volcanic ash clouds in the 10-12 $\mathrm{mm}$ window using AVHRR/2 data", Int. J. Remote Sensing, vol. 10, pp. 751-761, 1989.

[31] E. DeWayne Mitchell, Steven V. Vasiloff, Gregory J. Stumpf, Arthur Witt, Michael D. Eilts, and J. T. Johnson, Kevin W. Thomas, "The National severe 
storms laboratory tornado detection algorithm", Int. J. Remote Sensing, vol. 13, issue. 2, pp. 352-366, June 1998.

[32] Ilan Koren, Joachim H. Joseph, Peter Israelevich, "Detection of dust plumes and their sources in northeastern Libya", Can. J. Remote Sensing, vol. 29, no. 6, pp. 792-796, 2003.

[33] http : //hydrolab.arsusda.gov/rsbasics/RS $S_{S} y$ stem2.jpg

[34] http://noaasis.noaa.gov./NOAASIS/ml/AVHRR.html/

[35] http : //en.wikipedia.org/wiki/k - meansalgorithm

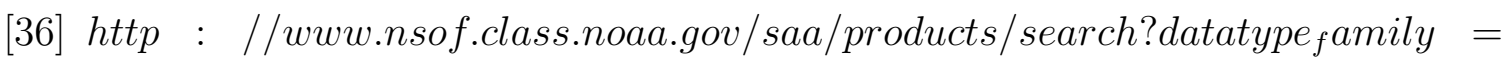
$A V H R R$

[37] http://www.hydrolab.arsusda.gov/rsbasics/noise.php

[38] http://www.ncdc.noaa.gov/oa/pod-guide/ncdc/docs/klm/index.htm

[39] http://www.dnr.sc.gov/ael/personals/pjpb/lecture/spectrum.gif 


\section{Appendix A}

\section{Discussion of MATLAB}

\section{Implementation}

The automation technique is implemented in MATLAB using functions of the Image Processing Toolbox. All the processing methods included in the automation technique like power spectrum analysis, binary thresholding and morphological enhancements are implemented using block processing.

Figure A.1 provides an overview of the automation technique for detecting dust storm.

Following is a brief explanation of the MATLAB functions used in the automation technique:

- blkproc: This function is used to processes the image by applying the specified function to each distinct block of the image. The block size mxn can be chosen by the user. Further processing is done on the blocks of this size. Overlapping blocks can also be chosen.

- fft2: This function computes the two-dimensional discrete Fourier transform (DFT), computed with a Fast Fourier Transform (FFT) algorithm. The trans- 


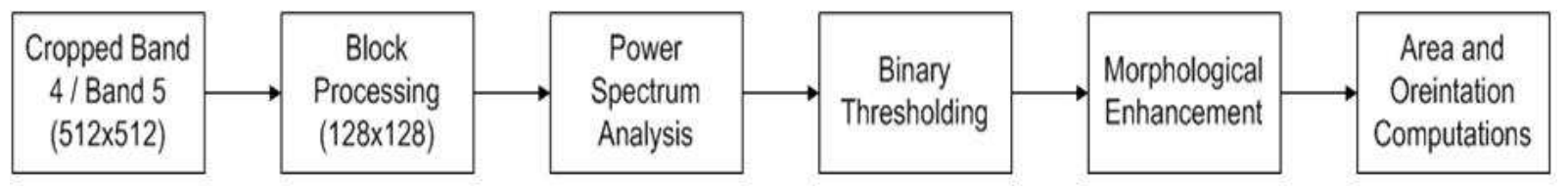

Figure A.1: Block diagram for Automated dust storm detection

form result is of the same size as the input. The output of any size mxn can be computed by truncating or zero padding the input array.

- graythresh: The graythresh function uses Ostu's thresholding method to convert the gray level image to binary image. The threshold is chosen in such a way so as to minimize the intraclass variance of the black and white pixels.

- imclose: This function performs morphological closing on the grayscale or binary image based on the chosen structuring element. The structuring element must be a single structuring element object, as opposed to an array of objects. strel is used to select the shape and size of the structuring element. The morphological close operation is a dilation followed by an erosion, using the same structuring element for both operations. The return value for this function is the closed image. 
- regionprops: This command is used to measure a set of properties for each labeled region in the label matrix. If properties is the string 'all', regionprops computes all the measurements. Different properties like centroid, orientation, bounding box, solidity, eccentricity can also be measured. In this research, we measure two properties of the binary images i.e., area and orientation. Area returns the number of pixels. Orientation measures the angle of the least inertia. An ellipse formed by the pixels in the binary image and the axes are determined. The angle is measured in degrees ranging from -90 to 90 degrees) between the $\mathrm{x}$-axis and the major axis of the ellipse that has the same second-moments as the region. 


\section{Curriculum Vitae}

Swapna Janugani was born on February 7, 1986 in Hyderabad, Andhra Pradesh, India. She is the eldest daughter of Rajeshwar Janugani and Nagamani Janugani. She did her schooling with Vivekananda High School, Hyderabad, India. She graduated from Jawaharlal Nehru Technological University (Autonomous), Hyderabad, India with a Bachelors degree in Electronics and Communication Engineering in June 2006. While pursuing Bachelors degree, she worked on a project at the National Remote Sensing Agency (NRSA), towards the development of a generalized algorithm for synthesizing Natural Color Composite Images for Indian Remote Sensing Satellites. From June 2006 to January 2007, she worked as an Assistant Systems Engineer for Tata Consultancy Services, India.

In the Spring of 2007, she entered the University of Texas at El Paso to pursue her Masters in Electrical Engineering. Her areas of specialization include Digital Signal and Image Processing. She began her research work under the guidance of Dr.Sergio.D.Cabrera. While at UTEP, she worked as a Graduate Assistant for the School of Nursing. During the summer of 2008, she worked as a Software Engineer Intern for Digital Health Group, Intel Corporation, Oregon. She graduated with Masters in Fall 2008. She is going to pursue her career as a Software Engineer with EchoStar Technologies in Denver, CO. Email ID : swapnajanugani@yahoo.com

This thesis was typeset with $\mathrm{AT}_{\mathrm{E} X}$ by the author. 